\title{
Adsorption Parameters and Phase Behaviour of Non-Ionic Surfactants at Liquid Interfaces
}

\author{
Radomir Iliev Slavchov ${ }^{1 *}$, Ivan Boyanov Ivanov ${ }^{2}$ \\ ${ }^{1}$ Department of Chemical Engineering and Biotechnology, Cambridge University, UK, CB3 OAS Cambridge, \\ Philippa Fawcett Drive, West Site \\ E-mail: ris26@cam.ac.uk \\ ${ }^{2}$ Laboratory of Chemical Physics and Engineering, Faculty of Chemistry and Pharmacy, Sofia University, 1164 \\ Sofia, Bulgaria \\ E-mail: ii@lcpe.uni-sofia.bg
}

\begin{abstract}
A reasonable adsorption model is one that allows all adsorption parameters (adsorption constant, hard-disc area $\alpha$, attraction parameter $\beta$ ) of a surfactant at a liquid interface to be predicted accurately as function of molecular structure and medium conditions. However, the established adsorption models of van der Waals and Frumkin lead to inconsistencies, such as negative $\beta$ at water|oil, $\alpha$ significantly larger than the crystallographic area of the molecule, and phase behaviour that contradicts the experimental observations. Several less popular models that are better suited for liquid interfaces are investigated. It is shown that the sticky disc model agrees with the observed adsorption behaviour of several homologous series of surfactants, both at water|air and water|oil interfaces. The area $\alpha$ is independent of the interface and agrees within $6 \%$ to what follows from collapse and crystallographic data. A model of the lateral attraction is proposed, from which it follows that $\beta$ has strongly non-linear dependence on the hydrocarbon chain length, the area of the head group and the temperature. Using the model of $\beta$, experimental data, and the law of corresponding states, the critical point of the adsorbed layer could be determined. Depending on the value of $\beta$, the adsorption behaviour of the surfactants at liquid interfaces can be classified into distinct categories: cohesive or non-cohesive, based on their Boyle points (where $\beta=2$ ), and sub-critical or super-critical, based on their critical points (where $\beta=38.1$ ).
\end{abstract}

Keywords: adsorption; non-ionic surfactant; steric repulsion; liquid expanded; attraction parameter. 


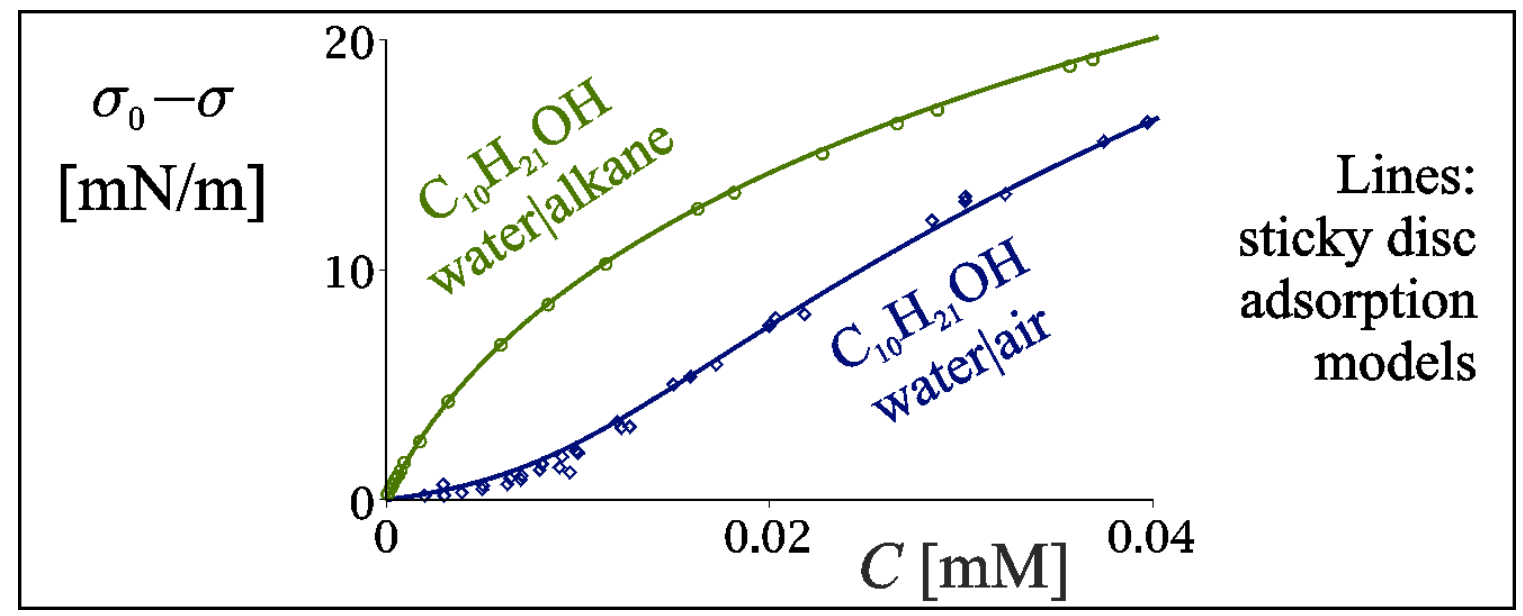

Graphical abstract

\section{Introduction}

The interest toward the relationship between surfactant structure and surface activity is constantly increasing [1-8]. A large amount of experimental data for the adsorption of surfactants at liquid surfaces, water|air (W|A) or water|oil (W|O), has been accumulated [1]. New techniques such as dynamic tensiometry, optical techniques, X-ray diffraction, etc., allowed better understanding of the properties of soluble surfactants [9-13]. This has been an impetus for new theoretical developments: novel mechanistic models of adsorption [14-18,6] were proposed, which are more suitable for liquid interfaces than the traditional models, based on Langmuir or Volmer isotherm. The adsorption parameters of these models were related to both surfactant structure and medium properties [6-8].

The classical studies of adsorption dealt mostly with isotherms of insoluble surfactants (obtained with the Langmuir trough technique [19-21]) relating surface pressure $\pi^{\mathrm{S}}$ and area per molecule $a$. These works revealed the complex phase behaviour of the monolayers. The phase diagram and the empirical equations of state (EOS) found by these studies resemble the phase behaviour of normal 3-dimensional (3-D) matter [22]. Adam [22,19] noticed that in the region between the gaseous and the solid-like state of the insoluble monolayers, there is a state of intermediate compressibility, which was called "liquid expanded (LE) state". Davies named the respective monolayers "cohesive" [23,24]. Langmuir concluded that the state of a LE monolayer is structureless, liquid-like [20]; direct evidence for that was later given [10]. The gaseous-LE phase transition was investigated in detail by Kim and Cannell [25], who 
determined the critical point for undissociated pentadecanoic acid.

The main source of experimental information for the adsorption of soluble surfactants at liquid interfaces is the dependence of the interfacial tension $\sigma$ on the surfactant bulk concentration $C[1,21]$. Tensiometric data for soluble monolayers are more difficult for interpretation than the $\pi^{S}(a)$ isotherms for insoluble surfactants. Indeed, it is difficult to distinguish between $1^{\text {st }}$ order phase transition from gaseous to liquid expanded monolayer (corresponding to a break of the $1^{\text {st }}$ derivative of $\sigma(C)$, cf. S10) and supercritical transition (corresponding to an inflection point) [5,6]. The techniques for direct observation of the surface phase transition, such as Brewster angle microscopy [26] and fluorescence microscopy [27], are harder to apply to soluble monolayers. Only recently the gaseous-to-LE transition was observed directly with soluble surfactants [9].

The study of the adsorption of non-ionic surfactants at $W \mid O$ interface was lagging behind $\mathrm{W} \mid \mathrm{A}$, since non-ionic surfactants are soluble in the oil phase. This makes Langmuir trough unusable (with few exceptions [28]). The adsorption is complicated by the accompanying processes of partitioning and surfactant association in the oil phase [29-32]. On the other hand, $\mathrm{W} \mid \mathrm{O}$ is the more interesting interface in technology, as water-oil emulsions are wide-spread in petroleum [33,34] and food [34] industry. The adsorption of resins and asphaltenes at $\mathrm{W} \mid \mathrm{O}$ is a key question in processes such as water flooding used to enhance oil recovery [34,35], and in water separation techniques [33]. In automotive engines, the lubricant is constantly diluted with water produced by the combustion process; the water-in-lubricant emulsion must be stabilized by the dispersants and detergents in the lubricant to decrease droplet size and avoid wear [36]. In fuel, even traces of water droplets have adverse effects on the operation of engines, causing corrosion [37], salty deposits [38] and increased risk of sparks [39]; water filters are present in the fuel delivery system to separate water droplets stabilized by non-ionic amphiphilic fuel components.

The most widely used approach in the theory of the adsorbed layers is based on the analysis of the famous van der Waals EOS (adapted from the theory of bulk phase equilibria, cf. e.g. Refs. [40,41]). Its main assumption is that there is a continuous transition between the two phases involved (gas and liquid), which can be described by a single EOS (a construction first proposed by Thomson [42]). We will refer to this approach as continual. This approach is usually used for soluble monolayers $[1,5,14-16]$ (and rarely for insoluble). All of the most popular equations of state for soluble monolayers - van der Waals, Frumkin - are of the continual type. Another approach is to treat each phase which is stable under given conditions as a separate entity with an equation of state of its own. We call this approach partial. The partial EOS is generally assumed valid only in the interval between the two transition points confining 
the respective phase state. This approach was used for defining the common EOS of insoluble monolayers [19,20,43].

The constitutive relations of the adsorption layer involve a set of adsorption parameters, which are functions of the surfactant structure and the medium properties. The most important parameter is the adsorption constant $K_{\mathrm{a}}$, related to the change of Gibbs energy of the molecule upon its transfer from the bulk of the solution to the interface. The other parameters are related to the lateral interactions between the molecules in the monolayer (actual area per molecule $\alpha$, attraction constant $\beta$ ). A key problem in the investigations of adsorption is the analysis of the dependence of these parameters on surfactant's structure (length $n$ of the alkyl chain, structure of the head, etc.), the interface type $(\mathrm{W}|\mathrm{A}, \mathrm{W}| \mathrm{O})$ and the medium properties (composition of the oil phase, temperature, etc.).

The adsorption constant $K_{\mathrm{a}}$ is by far the best studied adsorption parameter [44-47,24,6]. A useful theory of $K_{\mathrm{a}}$ was recently advanced by our group [46,7,8] which allows predicting the absolute value of $K_{\mathrm{a}}$. The correct interpretation of the interaction parameters $\alpha$ and $\beta$ turned out to be strongly dependent on the use of a correct adsorption isotherm for the analysis of experimental tensiometric data. The use of the EOS of Langmuir or Volmer (which are only qualitatively correct) allows some qualitative trends of the dependence of their $\alpha$ and $\beta$ parameters on the conditions to be revealed, but the absolute values of the parameters cannot be reliably predicted [3,7]. In some cases, the use of unsuitable isotherm might even lead to inconsistent results [7]. A physically sensible model should be able not only to fit the experimental data, but all parameters involved should be related appropriately to molecular structure. It will be shown here that, if one uses EOS and adsorption isotherm with firm theortical basis, all adsorption parameters involved can be calculated by means of molecular models without adjustable parameters.

Our ultimate task here is to test the performance of several continual models against tensiometric data at both $\mathrm{W} \mid \mathrm{A}$ and $\mathrm{W} \mid \mathrm{O}$ interface, and to provide recipes allowing the prediction of all main macroscopic characteristics of a monolayer. This involves, first, the qualitative characteristics such as cohesive or non-cohesive, supercritical or subcritical behaviour; and second, the quantitative prediction of all involved adsorption parameters and their dependence on the surfactant structure and the medium conditions. In a companion paper, we will compare these results with those obtained by the lesser-known partial equations of state, which are better suited for long-chained surfactants at W|A.

We begin by presenting briefly in Sec. 1.1 the concept of cohesive and non-cohesive behaviour and formulating criteria for distinguishing between them. In Sec. 2.1 we review critically the basic models of the adsorbed layer (we call "adsorption model" the combination of EOS and adsorption isotherm), 
including the unpopular but very reasonable hard disc model of Helfand, Frisch and Lebowitz [48] and the sticky disc model of Ivanov et al. [15,14,7]. Our theory of the adsorption constant $K_{\mathrm{a}}$ is presented in Sec. 2.2. A formula for the controversial attraction parameter $\beta$ is derived in Sec. 2.3. Procedures for experimental determination (both direct and from fit by an experimental model) of the adsorption parameters $K_{\mathrm{a}}, \alpha$ and $\beta$ are developed (in Sec. 3), and applied to several several homologous series of wide-spread non-ionic amphiphiles: alcohols, acids (which are of interest as model degradation products of the autooxidation of diesel and lubicants [49]), $\mathrm{N}$-alkylglycines (used as corrosion inhibitors in fuels and lubricants), and alkylphosphinoxides (which exhibit a pronounced transition from non-cohesive to cohesive behaviour with the increase of their chain length [8]). The phase behaviour of the adsorbtion layer is investigated in Sec. 3.6.

\subsection{Phenomenology of the effect of lateral intermolecular interaction on the adsorption behaviour: non-cohesive and cohesive monolayers}

The tensiometric data for various soluble surfactants at $\mathrm{W} \mid \mathrm{A}$ and $\mathrm{W} \mid \mathrm{O}$ interfaces can be divided into two classes: cohesive and non-cohesive isotherms [8]. Examples of those are given in Fig. 1 with the experimental dependence of the surface/interfacial pressure $\pi^{S}$ on concentration $C$ of decanol in the water phase for $\mathrm{W} \mid \mathrm{O}$ and $\mathrm{W} \mid \mathrm{A}$ interfaces $\left(\pi^{\mathrm{S}} \equiv \sigma_{0}-\sigma\right.$, where $\sigma_{0}$ is the surface/interfacial tension of the pure interface). Solid circles stand for the $\mathrm{W} \mid \mathrm{O}$ experimental data of Aveyard et al. [29-31] processed as described in S3. Empty circles and squares are data for W|A from Refs. [50-53].

At $\mathrm{W} \mid \mathrm{O}$, the interfacial tension is a function of decanol concentration with monotonous $1^{\text {st }}$ derivative; the $\pi^{\mathrm{S}}(C)$ isotherm begins with a linear (Henry's) region at low concentration and then gradually bends with the increase of $C$, without inflection point - we call such behaviour non-cohesive. The isotherm is convex; the negative deviation from ideality is a sign of predominance of repulsive forces over lateral attraction between the molecules in the adsorption layer.

At W|A and low concentration of decanol, there is a Henry's region where $\pi^{\mathrm{S}}$ is a linear function of $C$ with zero intercept (Fig. 1, line "gaseous"). At certain concentration, the slope abruptly increases we call this kink point of transition. The behaviour after the kink is also approximately linear, but with negative intercept (designated with $\pi_{\text {coh }}$ in Fig. 1). At still higher concentrations the isotherm becomes convex similarly to that at $\mathrm{W} \mid \mathrm{O}$. We call such behaviour cohesive. The kink may indicate phase transition or a sharp supercritical transition between two phases, cf. S10. We will show in Sec. 3.6 that for all surfactants studied by us this transition is most likely supercritical. The region before the kink 
corresponds to gaseous monolayer, while the features of the region after the kink are indicative of liquid expanded (LE) state [6,8]. The intercept $\pi_{\mathrm{coh}}$ for soluble surfactants is giving the so-called cohesive (spreading) pressure introduced by Langmuir [20,6,8] for insoluble monolayers.

The kink can be observed only if enough data are available at the lowest concentration in the gaseous region. Often, due to the absence of data in the Henry's region, one has to use other criteria for distinguishing between cohesive and non-cohesive behaviour. A negative intercept of the initial portion of the $\pi^{\mathrm{S}}(C)$ curve after the kink is indicative of cohesive interaction - this can be used as a second criterion for existence of the LE state [8]. A third criterion is the value of the adsorption energy $\Delta \mu_{\mathrm{CH}_{2}}$ of a $-\mathrm{CH}_{2}-$ group. Since in the LE state the adsorbed molecules are in a oil-like environment, $\Delta \mu_{\mathrm{CH}_{2}}$ has the same value, $1.39 \times k_{\mathrm{B}} T$, as for $\mathrm{W} \mid \mathrm{O}$ interface, in contrast to the adsorption energy in the gaseous region which coincides with the known value of $\Delta \mu_{\mathrm{CH}_{2}}=1.04 \times k_{\mathrm{B}} T$ for dilute adsorption layers at W|A [8].

Usually, the $\pi^{\mathrm{S}}(C)$ isotherms at $\mathrm{W} \mid \mathrm{O}$ are non-cohesive. $\mathrm{W} \mid \mathrm{A}$ isotherms are cohesive, except for surfactants of short hydrophobic chain which behave non-cohesively even at W|A.

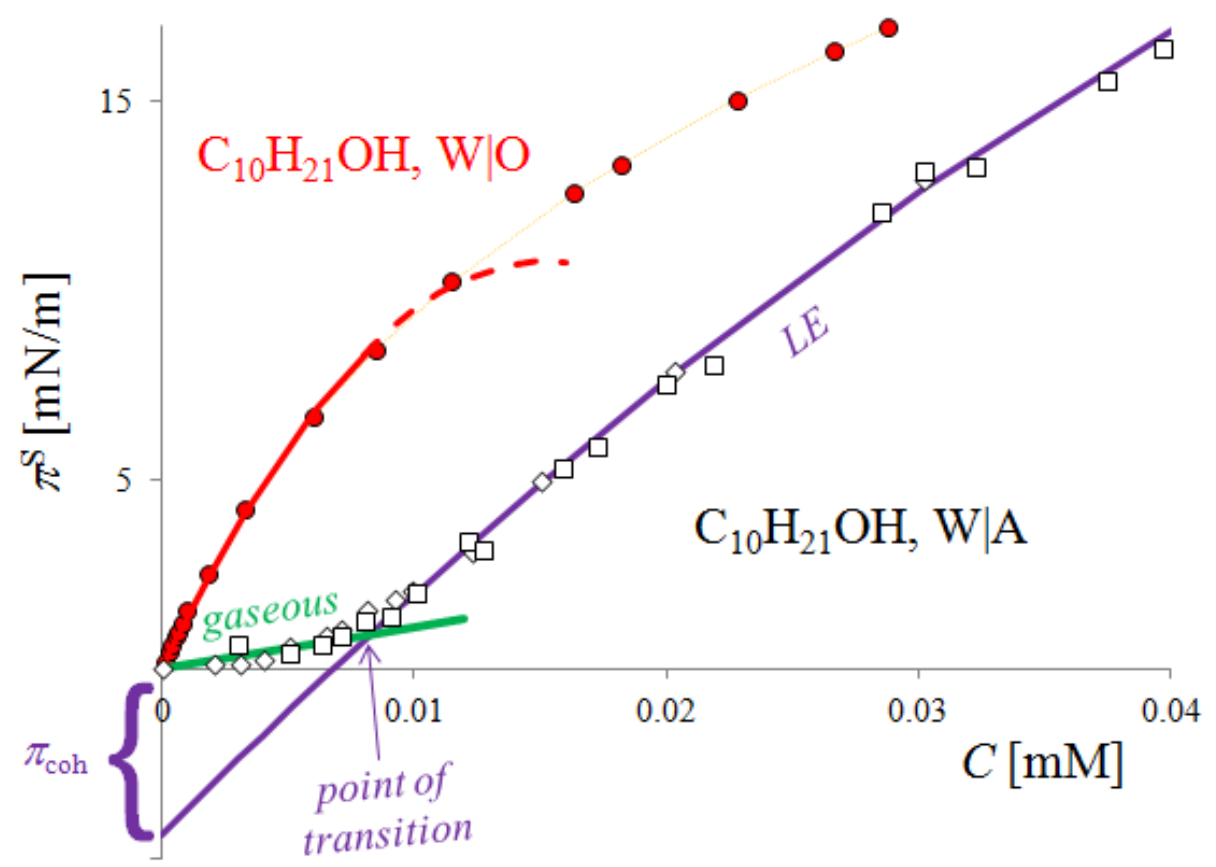

Fig. 1. Comparison between the surface and interfacial pressure isotherms, $\pi^{\mathrm{S}}$ vs. decanol concentration $C$ in water, at $\mathrm{W} \mid \mathrm{A}$ and $\mathrm{W} \mid \mathrm{O}$ interfaces. This surfactant behaves non-cohesively at $\mathrm{W} \mid \mathrm{O}$ and cohesively at $\mathrm{W} \mid \mathrm{A}$. Data from Refs. [29-31,50-53] have been used. The W|O line is a quadratic fit with the virial expansion (6), cf. Sec.

3.1 for details; the other lines are to guide the eye. 


\section{Adsorption models for surfactants at liquid interfaces}

\subsection{Basic continual models of the adsorbed layer}

\subsubsection{Hard-disc fluid}

As mentioned in Sec. 1.1, the deviation from ideality observed with the non-cohesive adsorption layers is due to the predominance of the repulsive forces over attraction. Therefore, one may expect that all main features of the non-cohesive behaviour will be grasped by a model based on two-dimensional hard disc fluid. A simple, nearly exact equation of state (EOS) of such fluid was derived by Helfand, Frisch and Lebowitz (HFL) [48]:

$$
\pi^{\mathrm{s}} / k_{\mathrm{B}} T=\Gamma /(1-\alpha \Gamma)^{2} \quad(H F L),
$$

where $\Gamma$ is adsorption, $\alpha$ is the actual area of the adsorbed molecule, $k_{\mathrm{B}}$ is Boltzmann constant, $T$ is temperature. From the EOS (1) and the Gibbs isotherm, $\mathrm{d} \pi^{\mathrm{S}}=\Gamma \mathrm{d} \mu^{\mathrm{S}}$, one can obtain the dependence of the chemical potential $\mu^{\mathrm{S}}$ of the surfactant in the adsorbed layer on the adsorption $\Gamma$ :

$$
\begin{aligned}
& \mu^{\mathrm{S}}=\mu_{0}^{\mathrm{S}}+k_{\mathrm{B}} T \ln \gamma^{\mathrm{S}} \Gamma, \text { where } \\
& \ln \gamma^{\mathrm{S}}=-\ln (1-\alpha \Gamma)+\alpha \Gamma(3-2 \alpha \Gamma) /(1-\alpha \Gamma)^{2} .
\end{aligned}
$$

Here $\gamma^{S}$ is the surface activity coefficient and $\mu_{0}^{S}$ is the standard chemical potential of the surfactant at the interface. Eq. (2) was obtained by Buff and Stillinger who used it in their model of ions in the Helmholtz layer at an electrode surface (Eqs. 41\&42 of Ref. [54]). The adsorption isotherm of a nonideal adsorption layer can be obtained by setting in (2) $\mu^{\mathrm{S}}=\mu^{\mathrm{B}}$, where $\mu^{\mathrm{B}}=\mu_{0}^{\mathrm{B}}+k_{\mathrm{B}} T \ln C$ is the chemical potential of the surfactant in the bulk solution and $\mu_{0}^{\mathrm{B}}$ is the respective standard potential. This condition for equilibrium yields:

$$
K_{\mathrm{a}} C=\gamma^{\mathrm{S}} \Gamma, \quad \text { where } \quad K_{\mathrm{a}}=\exp \left[\left(\mu_{0}^{\mathrm{B}}-\mu_{0}^{\mathrm{S}}\right) / k_{\mathrm{B}} T\right] .
$$

Here, $K_{\mathrm{a}}$ is the adsorption constant of the surfactant. Substituting $\gamma^{\mathrm{S}}$ from Eq. (2) into Eq. (3), one obtains the HFL adsorption isotherm:

$$
K_{\mathrm{a}} C=\frac{\Gamma}{1-\alpha \Gamma} \exp \left[\frac{\alpha \Gamma(3-2 \alpha \Gamma)}{(1-\alpha \Gamma)^{2}}\right] .
$$

This isotherm was derived by Ivanov et al. [46]. For a non-ideal bulk solution, the concentration $C$ in 
Eqs. (3)-(4) must be replaced by the bulk activity of the surfactant, $\gamma C$, where $\gamma$ is the bulk activity coefficient. Eqs. (1)\&(4) define parametrically (with parameter $I$ ) the surface pressure isotherm $\pi^{\mathrm{S}}(C)$ corresponding to the Helfand-Frisch-Lebowitz model.

Other equations of state for two-dimensional hard-disc fluid have been extensively used in the literature. The most popular are those of Langmuir (which is rigorously valid only for localized adsorption at solid surfaces $[55,56]$ ) and of Volmer (which is rigorously valid only for fluid of hard rods adsorbed on a line [57,58]):

$$
\begin{aligned}
& \pi^{\mathrm{S}} / k_{\mathrm{B}} T=-\alpha_{\mathrm{L}}^{-1} \ln \left(1-\alpha_{\mathrm{L}} \Gamma\right) \quad \text { (Langmuir); } \\
& \pi^{\mathrm{S}} / k_{\mathrm{B}} T=\Gamma /\left(1-\alpha_{\mathrm{v}} \Gamma\right) \quad(\text { Volmer }) .
\end{aligned}
$$

When applied to 2-D fluid, these EOS account only approximately for the effect of the area parameter $\alpha$ on the adsorption behaviour, i.e. on the functions $\pi^{\mathrm{S}}(\Gamma)$ and $\gamma^{\mathrm{S}}(\Gamma)$. Comparison of these equations and their extensions for attracting particles (the EOS of Frumkin [59] and van der Waals [60,61]) with tensiometric data usually yield only the correct order of magnitude for the molecular areas of various surfactants [1,5]. However, the obtained values of the respective area parameters $\alpha_{\mathrm{L}}$ and $\alpha_{\mathrm{V}}$ differ from those obtained from molecular geometry or crystallographic data $[7,8]$.

The HFL model is particularly convenient for adsorption at $\mathrm{W} \mid \mathrm{O}$, where the lateral attraction between hydrocarbon chains is small $[62,8]$ and can be usually disregarded. HFL model can be also used as a first approximation for non-cohesive isotherms at $\mathrm{W} \mid \mathrm{A}[8]$, but since in this case the attractive forces are non-negligible, the value of the area $\alpha$ obtained with HFL is by few $\AA^{2}$ smaller than the one following from, e.g., crystallographic data [8].

\subsubsection{Continual models involving lateral attraction}

The simplest model accounting for the lateral interaction in the adsorption layer is the virial expansion of the surface pressure isotherm $\pi^{\mathrm{S}}(C)$, which can be represented as

$$
\pi^{\mathrm{s}} / k_{\mathrm{B}} T=K_{\mathrm{a}} C-B_{2}\left(K_{\mathrm{a}} C\right)^{2} \quad \text { (virial expansion). }
$$

Here $B_{2}$ is the second virial coefficient. This equation follows from the standard virial expansion $\pi^{\mathrm{S}} / k_{\mathrm{B}} T$ $=\Gamma+B_{2} \Gamma^{2}$ and the corresponding adsorption isotherm ${ }^{1}$. According to Eq. (6), if $B_{2}>0$ (repulsion is dominating), negative deviations from ideality in the $\pi^{\mathrm{S}}(C)$ isotherm will occur (as in Fig. 1, W|O data).

The most widely used models taking into account the attractive interactions between the adsorbed molecules are the Frumkin and the two-dimensional van der Waals model ${ }^{2}$ (indexed "vdW" below). 
Frumkin [59], Volmer and Mahnert [60] and later - de Boer [61] corrected empirically the hard-disc EOS (5), with the addition of an attractive term, $-\beta_{\mathrm{L}} \alpha \Gamma^{2}$ to Langmuir's and $-\beta_{\mathrm{V}} \alpha \Gamma^{2}$ to Volmer's EOS:

$$
\begin{aligned}
& \frac{\pi^{\mathrm{S}}}{k_{\mathrm{B}} T}=-\frac{1}{\alpha_{\mathrm{L}}} \ln \left(1-\alpha_{\mathrm{L}} \Gamma\right)-\beta_{\mathrm{L}} \alpha_{\mathrm{L}} \Gamma^{2} \quad \text { (Frumkin) } \\
& \frac{\pi^{\mathrm{S}}}{k_{\mathrm{B}} T}=\frac{\Gamma}{1-\alpha_{\mathrm{v}} \Gamma}-\beta_{\mathrm{v}} \alpha_{\mathrm{v}} \Gamma^{2} \quad(v d W) .
\end{aligned}
$$

Smith [63] did the same with the HFL EOS (1) to obtain:

$$
\frac{\pi^{\mathrm{S}}}{k_{\mathrm{B}} T}=\frac{\Gamma}{(1-\alpha \Gamma)^{2}}-\beta \alpha \Gamma^{2} \quad(\text { SIAL }) .
$$

Ivanov et al. [46] used Eq. (8) along with Gibbs isotherm, to derive the corresponding adsorption isotherm:

$$
K_{\mathrm{a}} C=\frac{\Gamma}{1-\alpha \Gamma} \exp \left[\frac{\alpha \Gamma(3-2 \alpha \Gamma)}{(1-\alpha \Gamma)^{2}}-2 \beta \alpha \Gamma\right] .
$$

We refer to Eqs. (8)-(9) as to Smith-Ivanov-Ananthapadmanabhan-Lips (SIAL) model.

The correction of the hard-disc EOS for the attractive forces by the addition of a term of the type$\beta \alpha \Gamma^{2}$ is valid for binary interactions (i.e. at low surface density [64]), but it is by no means justified for dense monolayers. A more consistent approach to the attractive part of the EOS was proposed by Ivanov et al. $[15,14,7]$. These authors were able to derive an exact equation of state for 1-D gas of hard rods with sticky attractive potential, which accounts correctly both for attraction and repulsion in the 1-D case at any surface coverage [14]. Ivanov et al. further extended their 1-D results to the 2-D case ${ }^{3}$ by deriving a new EOS in which a term for the intermolecular attraction, similar to that for the 1-D system, is combined with a repulsive factor $\Pi(1-\alpha)^{2}$ identical to HFL surface pressure [14]. The final result was the following 2-D EOS:

$$
\frac{\pi^{\mathrm{S}}}{k_{\mathrm{B}} T}=\frac{\Gamma}{(1-\alpha \Gamma)^{2}} \times \frac{2}{1+R_{\beta}}=\frac{R_{\beta}-1}{2 \alpha \beta(1-\alpha \Gamma)} \quad(S D),
$$

where $R_{\beta}$ stands for the expression

$$
R_{\beta}=\sqrt{1+16 \beta_{\text {contact }} \frac{\alpha \Gamma}{1-\alpha \Gamma}}=\sqrt{1+4 \beta \frac{\alpha \Gamma}{1-\alpha \Gamma}} .
$$

Here $\beta_{\text {contact }}$ is the contact parameter of the sticky potential, used by Ivanov et al. [15], which is related simply to the attraction parameter: $\beta=4 \beta_{\text {contact }}$ [7]. By using the Gibbs adsorption isotherm, the corresponding isotherm was derived: 


$$
K_{\mathrm{a}} C=\frac{\Gamma}{1-\alpha \Gamma}\left(\frac{2}{1+R_{\beta}}\right)^{2+1 / \beta} \exp \left[\frac{\alpha \Gamma(4-3 \alpha \Gamma)}{(1-\alpha \Gamma)^{2}} \times \frac{2}{1+R_{\beta}}\right] .
$$

Eqs. (10)-(12) will be referred to as sticky disc model (SD) below.

The SIAL and the SD models have the advantage to predict correctly the theoretical second virial coefficient, $B_{2}=2 \alpha-\alpha \beta$ (cf. Sec. 2.3 below). In addition to this, the SD model, Eqs. (10)-(12), has a value of the third virial coefficient that is nearly exact in comparison with that of a sticky disc 2-D fluid [15]. Comparison with experimental data for undissociated acids and alkylphosphineoxides has been made [15]. Eqs. (10)-(12) were generalized to the case of ionic surfactants, and compared to data for dodecylsulfate and dodecyltrimethylammonium salts [7].

The EOS and the adsorption isotherms of the continual models discussed in this section are summarized in the S1, Table S1.

\subsection{The adsorption constant $K_{\mathrm{a}}$}

The adsorption constant $K_{\mathrm{a}}$ was related to the surfactant structure and the parameters of the media (temperature, bulk compositions etc.) by Ivanov et al. $[46,7,8]$ who proposed a model of the interaction free energy ${ }^{4} \Delta \mu(z)$ of a surfactant molecule with the interface. The final formula for $K_{\mathrm{a}}$ reads:

$$
K_{\mathrm{a}}=\delta_{\mathrm{a}} \exp \left(E_{\mathrm{a}} / k_{\mathrm{B}} T\right),
$$

where $\delta_{\mathrm{a}}$ is the adsorption length, and $E_{\mathrm{a}}$ is the adsorption free energy. For readers' convenience, the derivation of this formula, together with a summary of the features of the model, is presented in S2. The result for the adsorption length reads:

$$
\delta_{\mathrm{a}}=l_{\mathrm{CH}_{2}} k_{\mathrm{B}} T / 2 \Delta \mu_{\mathrm{CH}_{2}}
$$

here $\Delta \mu_{\mathrm{CH}_{2}}$ is the change of the free energy of a single $-\mathrm{CH}_{2}-$ group upon its transfer from the hydrophobic phase to the water phase $\left(\Delta \mu_{\mathrm{CH}_{2}}>0\right)$ and $l_{\mathrm{CH}_{2}}$ is the length of a $-\mathrm{CH}_{2}-$ group along the hydrophobic chain $\left(l_{\mathrm{CH}_{2}}=1.26 \AA[62,65]\right)$. The adsorption free energy stands for the expression:

$$
E_{\mathrm{a}}=(n-1) \Delta \mu_{\mathrm{CH}_{2}}+\Delta \mu_{\mathrm{CH}_{3}}+\alpha_{\perp} \sigma_{0}+\Delta \mu_{\text {head }} .
$$

Here, $(n-1) \Delta \mu_{\mathrm{CH}_{2}}+\Delta \mu_{\mathrm{CH}_{3}}$ is the contribution of the hydrophobic solvation of the surfactant's linear hydrocarbon chain ( $n$ is the number of the carbon atoms in it, and $\Delta \mu_{\mathrm{CH}_{3}}$ is the transfer energy of a $-\mathrm{CH}_{3}$ group). The term $-\alpha \perp \sigma_{0}$ was introduced in Refs. [46,7,6]. It accounts for the fact that upon adsorption, the hydrocarbon chain penetrates the interface which leads to disappearance of a portion of pure interface of interfacial tension $\sigma_{0}$ and area $\alpha_{\perp}$ (close to the crystallographic area of the chain). Finally, 
the empirical constant $\Delta \mu_{\text {head }}$ encompasses several effects: (i) The interaction between the hydrophilic head group and the interface. (ii) Possible appearance of an induced dipole moment in the $-\mathrm{CH}_{2}-$ group adjacent to the polar hydrophilic head, which acts oppositely to the hydrophobic effect and leads to immersion of the methylene group into the water phase (cf. chap. 3 of Ref. [65]). (iii) Changes in the internal degrees of freedom (vibration and internal rotation) of the molecule upon adsorption. The last effect is involved to a certain extent in the transfer energies $\Delta \mu_{\mathrm{CH}_{2}}$ and $\Delta \mu_{\mathrm{CH}_{3}}$ : since we are using experimental values for them, it is probably accounted for implicitly in our model. The contribution of the factors (i-iii) to $\Delta \mu_{\text {head }}$ will be analysed post-factum, by comparing theoretical results with the experimental data (Sec. 3.1).

The final result (13) for the adsorption constant, $K_{\mathrm{a}}=\delta_{\mathrm{a}} \exp \left(E_{\mathrm{a}} / k_{\mathrm{B}} T\right)$, formally coincides with the widely used formula of Davies and Rideal [24]. They assumed, however, that $\delta_{\mathrm{a}}$ is the length of the hydrophobic chain (which is very different from Eq. (14)), and disregarded the contributions of $\Delta \mu_{\mathrm{CH}_{3}}$ and the lost interface to $E_{\mathrm{a}}$. Ivanov's definition of the adsorption length $\delta_{\mathrm{a}}$ is similar to the one occurring in the theory of adsorption of spherical molecules at solid interfaces [56] in the sense that it involves only factors related to the kinetic energy (translation and rotation) of the adsorbed molecules.

\subsection{The attraction parameter $\beta$}

A rigorous expression for the second virial coefficient $B_{2}$ of 3-D fluid of hard spheres of radius $R$ interacting with each other with an attractive potential $u_{\text {attr }}(r)$ is given by Eq. 5.3 in Ref. [66]. Modifying this expression for the case of interacting hard discs, one obtains:

$$
B_{2}=-\pi \int_{0}^{\infty}\left(\mathrm{e}^{-u / k_{\mathrm{B}} T}-1\right) r \mathrm{~d} r=2 \alpha-\alpha \beta
$$

where $r$ is the distance between two particles, and $\alpha=\pi R^{2}$. The dimensionless quantity $\beta$ in Eq. (15) stands for the integral:

$$
\beta=\frac{1}{R^{2}} \int_{2 R}^{\infty}\left(\mathrm{e}^{-u_{\text {attr }} / k_{\mathrm{B}} T}-1\right) r \mathrm{~d} r .
$$

It is known in the literature as attraction parameter [1]. The $2 \alpha$ term in Eq. (15) is the repulsive harddisc part of the virial coefficient. Since Eq. (15) is an exact result of the statistical thermodynamics, any EOS based on hard discs models must be concordant with it. However, the expansions of the most popular Frumkin and van der Waals Eqs. (7) lead to different results [7], namely: 


$$
\begin{aligned}
& \pi^{\mathrm{S}} / k_{\mathrm{B}} T \rightarrow \Gamma+\left(\alpha_{\mathrm{L}} / 2-\alpha_{\mathrm{L}} \beta_{\mathrm{L}}\right) \Gamma^{2}+\ldots \quad(\text { Frumkin }) \text { and } \\
& \pi^{\mathrm{S}} / k_{\mathrm{B}} T \rightarrow \Gamma+\left(\alpha_{\mathrm{V}}-\alpha_{\mathrm{V}} \beta_{\mathrm{V}}\right) \Gamma^{2}+\ldots \quad(v d W), \text { i.e. } \\
& B_{2}=\alpha_{\mathrm{L}} / 2-\alpha_{\mathrm{L}} \beta_{\mathrm{L}} \quad \text { and } B_{2}=\alpha_{\mathrm{V}}-\alpha_{\mathrm{V}} \beta_{\mathrm{V}} .
\end{aligned}
$$

Following the classical statistical derivation of the 3 -dimensional van der Waals EOS ( $\$ 76$ of Landau and Lifshitz [64]), one can relate the $\alpha_{\mathrm{L}, \mathrm{V}}$ and $\beta_{\mathrm{L}, \mathrm{V}}$ parameters of Frumkin and the 2-dimensional van der Waals EOS (7) to the actual area $\alpha$ and the actual attraction parameter (16). This is usually done by setting equal separately the repulsive (hard-disc) and the attractive components of Eqs. (15)\&(17):

$$
\begin{array}{lll}
\alpha_{\mathrm{L}}=4 \alpha, & \beta_{\mathrm{L}}=\beta / 4 ; & \\
\alpha_{\mathrm{V}}=2 \alpha ; & \beta_{\mathrm{V}}=\beta / 2 . & (\text { Frumkin })
\end{array}
$$

According to Eqs. (18), the areas $\alpha_{\mathrm{V}}$ and $\alpha_{\mathrm{L}}$ differ from the real area of the molecule $\alpha$ and the same is valid for the attraction parameters (cf. also [63]). As pointed out by Landau and Lifshitz, the relations (18) are conditions for interpolation [64] but this interpolation is not unique. Indeed, instead of Eq. (18) , most authors assume that the values of $\alpha_{\mathrm{V}}$ or $\alpha_{\mathrm{L}}$, determined experimentally by using the respective EOS, are equal to the true geometrical area $\alpha=\pi R^{2}$ of the molecule (e.g. Refs. $\left.[1,2,4,5]\right)$. However, if one assumes that $\alpha_{\mathrm{L}}=\alpha$ or $\alpha_{\mathrm{V}}=\alpha$, then a different definition is needed also for $\beta_{\mathrm{L}}$ and $\beta_{\mathrm{V}}$ instead of Eq. (18). They must be defined so that the respective EOS yield the correct value of $B_{2}$ given by Eq. (15). Hence, setting $B_{2}$ from Eq. (15) equal to $B_{2}$ from Eqs. (17), one obtains:

$$
\begin{array}{lll}
\alpha_{\mathrm{L}}=\alpha ; & \beta_{\mathrm{L}}=\beta-3 / 2 & \text { (Frumkin); } \\
\alpha_{\mathrm{V}}=\alpha ; & \beta_{\mathrm{V}}=\beta-1 & (\text { vdW }) .
\end{array}
$$

Therefore, if $\alpha_{\mathrm{L}}=\alpha$ or $\alpha_{\mathrm{V}}=\alpha$ is assumed, the values of $\beta_{\mathrm{L}}$ and $\beta_{\mathrm{V}}$ turn out to be significantly lower (even negative) than the correct respective value of $\beta$ following from the definition (16). To the best of our knowledge, Eq. (19) has not been derived before. We will discuss it briefly, since it is important for the understanding of the physical meaning of the values of the parameters $\beta_{\mathrm{L}}$ and $\beta_{\mathrm{V}}$ obtained in the literature. The problems stemming from the definition (19) of $\beta_{\mathrm{V}}$ and $\beta_{\mathrm{L}}$ are best illustrated by the consideration of the adsorption of non-ionic surfactants at $\mathrm{W} \mid \mathrm{O}$ interfaces. Such systems must exhibit little or no lateral attraction, i.e. $u_{\mathrm{attr}} \approx 0$ [62]. According to Eq. (16), the true attraction parameter must then be $\beta \approx 0$. However, it follows from Eqs. (19) that the value $\beta=0$ corresponds to $\beta_{\mathrm{L}}=-3 / 2$ and $\beta \mathrm{V}$ $=-1$. For the similar case of weakly interacting non-cohesive surfactants at W|A, the EOS of Frumkin and van der Waals must also lead to negative values of $\beta_{\mathrm{L}}$ and $\beta \mathrm{V}$, while the true attraction parameter is small positive. This fact explains the "confusing" reports of some authors about negative values of $\beta_{\mathrm{L}}$ 
and $\beta_{\mathrm{V}}$ of some short-chain surfactants (e.g., Refs. [2,4], tables 3.6,3.8\&3.10 of Ref. [1], etc.).

Another important problem with the interpretation of the parameters of the EOS is that some authors [47], working with Frumkin or van der Waals EOS, use simultaneously Eqs. (18) and (19): for example, they assume that the area $\alpha_{\mathrm{L}}$ or $\alpha_{\mathrm{V}}$ is the actual area (as in Eq. (19)) but define the attraction parameter as $\beta_{\mathrm{V}}=\beta / 2$ as it is according to Eq. (18), which is not legitimate.

Further discussion on the relation between $\alpha_{\mathrm{V}}, \alpha_{\mathrm{L}}$ and the real area $\alpha$ can be found in Refs. [46,7,67]. Briefly, it was shown by Ivanov et al. [46,7] that the results (18) $\left(\alpha_{\mathrm{L}}=4 \alpha\right.$ and $\left.\alpha_{\mathrm{V}}=2 \alpha\right)$ are correct only at $\Gamma \rightarrow 0$, while the relations $\alpha_{\mathrm{L}}=\alpha \mathrm{v}=\alpha$, cf. Eqs. (19), are reached only at $\Gamma \rightarrow 1 / \alpha$.

Eq. (16) allows the calculation of $\beta$ provided that $u_{\text {attr }}(r)$ is known. A first approximation for $u_{\text {attr }}(r)$ was proposed in Ref. [46], based on the following assumptions: (i) At $r>2 R$, the adsorbed molecules interact with attractive London interaction, which is the sum of the London potentials $-L_{\mathrm{CH}_{2}} / \boldsymbol{r}_{1}-\left.\boldsymbol{r}_{2}\right|^{6}$ between the $-\mathrm{CH}_{2}-$ groups composing the hydrocarbon chain; $\boldsymbol{r}_{1}$ and $\boldsymbol{r}_{2}$ are the vector-positions of two $-\mathrm{CH}_{2}$ - groups and $L_{\mathrm{CH}_{2}}=4.24 \times 10^{-78} \mathrm{~m}^{6} \mathrm{~J}[62]$ is the respective London constant. (ii) The molecules are perpendicular to the interface. (iii) Water molecules are not involved in the interaction. (iv) The effect of the orientation of the $\mathrm{C}-\mathrm{C}$ and $\mathrm{C}-\mathrm{H}$ bonds on London's interaction [66] is neglected. The tails of the surfactant molecules were modelled as lines of uniform linear molecular density $1 / l_{\mathrm{CH}_{2}}$. Under these assumptions, $u_{\mathrm{attr}}(r)$ is the total interaction between the two hydrophobic tails; it is given by:

$$
u_{\text {attr }}(r)=-\int_{0}^{n l_{\mathrm{CH}_{2}}} \int_{0}^{n l_{\mathrm{CH}_{2}}} \frac{L_{\mathrm{CH}_{2}}}{\left[r^{2}+\left(z_{1}-z_{2}\right)^{2}\right]^{3}} \frac{\mathrm{d} z_{1} \mathrm{~d} z_{2}}{l_{\mathrm{CH}_{2}}^{2}}=-\frac{n L_{\mathrm{CH}_{2}}}{4 l_{\mathrm{CH}_{2}} r^{5}}\left(3 \arctan \frac{n l_{\mathrm{CH}_{2}}}{r}+\frac{n l_{\mathrm{CH}_{2}} r}{r^{2}+n^{2} l_{\mathrm{CH}_{2}}^{2}}\right) \text {, }
$$

where $z_{1}$ and $z_{2}$ are the vertical coordinates of the interacting $-\mathrm{CH}_{2}-$ groups. Similar model was used by Israelashvili for the computation of the heat of sublimation of alkanes (sec. 6.2 in Ref. [62]).

To calculate $\beta$, one substitutes into its definition (16) the expression (20) for $u_{\text {attr }}$ To facilitate the calculation, it is convenient to change the integration variable by setting $r=n l_{\mathrm{CH}_{2}} \tilde{r}$. The result is:

$$
\beta(\tilde{\alpha}, \tilde{T})=\frac{\pi}{\tilde{\alpha}} \int_{2 \sqrt{\tilde{\alpha} / \pi}}^{\infty}\left[\exp \left(\frac{3 \arctan \frac{1}{\tilde{r}}+\frac{\tilde{r}}{\tilde{r}^{2}+1}}{4 \tilde{T}^{5}}\right)-1\right] \tilde{r} \mathrm{~d} \tilde{r}
$$

where the dimensionless temperature and area are defined as:

$$
\tilde{T}=n^{4} l_{\mathrm{CH}_{2}}^{6} k_{\mathrm{B}} T / L_{\mathrm{CH}_{2}}, \quad \tilde{\alpha}=\alpha / n^{2} l_{\mathrm{CH}_{2}}^{2} .
$$

The integration of Eq. (21) is performed numerically and the results for $\beta$ as a function of $\alpha$ and $n$ are presented in Fig. 2. In Ref. [15], the expression (21) was used for surfactants with relatively large head 
groups. For this case, the exponent under the integral can be expanded into series up to the linear term. This leads to an analytical formula for $\beta$ [15]. However, even for short chain lengths, the linear expansion deviates significantly from the exact result (21) - this is demonstrated in S5. Therefore, when comparing the model for $\beta$ with experimental data, we will use only the exact Eq. (21).

Smith [63] used a different approach to calculate $\beta$. He represented the hydrophobic chains as stacks of $n$ small cylinders of length $l_{\mathrm{CH}_{2}}$ and assumed that each of them interacts only with its closest neighbour from the adjacent molecule. He considered the hydrophilic head group and the terminal $-\mathrm{CH}_{3}$ as being equivalent to one $-\mathrm{CH}_{2}-$ each; in other words, he assumed that

$$
\beta(n)=(n+1) \beta_{1} \text {. }
$$

He used the value $\beta_{1}=0.49$, obtained from the experimental second virial coefficient of $\mathrm{CH}_{4}$ [63]. The expression (23) for $\beta$ was used also by Kralchevsky et al. [68,5], but they determined the value of $\beta_{1}$ from the experimental data as a fitting parameter. A more realistic variant of this linear dependence of $\beta$ on $n$ would allow for intercept $\beta_{0} \neq \beta_{1}$ :

$$
\beta(n)=\beta_{0}+\beta_{1} n
$$

The intercept $\beta_{0}$ takes explicitly into account the head group and the terminal $-\mathrm{CH}_{3}$ group.

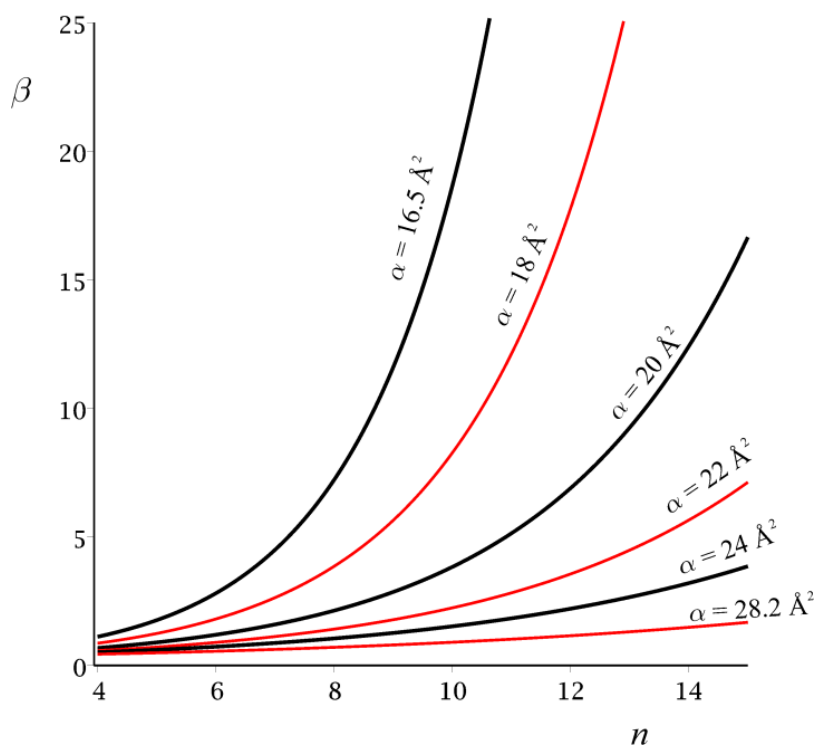

Fig. 2. The attraction parameter $\beta$ vs. the number of carbon atoms $n$ in the surfactant hydrophobic chain, calculated by numerical integration of Eq. (21), at various values of the actual area per molecule $\alpha$ (at $25^{\circ} \mathrm{C}$ ). The areas 16.5, 18 and 28.2 $\AA^{2}$ correspond to $\mathrm{C}_{n} \mathrm{H}_{2 n+1} \mathrm{OH}, \mathrm{C}_{n-1} \mathrm{H}_{2 n-1} \mathrm{COOH}$ and $\mathrm{C}_{n} \mathrm{H}_{2 n+1} \mathrm{Me} 2 \mathrm{PO}$, respectively. 


\section{Determination of the adsorption parameters from experimental data}

A fitting procedure with several free parameters may force the model curve to pass well through the experimental points but the obtained values of the parameters may be erroneous, even unphysical. In fact, all models formulated in Sec. 2.1 involve many approximations and consequently, no perfect coincidence of the theoretical predictions with tensiometric data should be expected. In addition, we found that although authors claim uncertainty of $\pm 0.3 \mathrm{mN} / \mathrm{m}$ or less for $\pi^{\mathrm{S}}$, comparison of data by different authors will rarely show coincidence with accuracy better than $\pm 1 \mathrm{mN} / \mathrm{m}$, and often it is about $\pm 2-3 \mathrm{mN} / \mathrm{m}$. Reasons are: impurities, slow adsorption and need for extrapolation toward equilibrium, systematic experimental deviations are typical for all common techniques; calibration-related deviations are possible. Therefore we consider dispersion $1 \mathrm{mN} / \mathrm{m}$ adequate and consider further optimization ungrounded considering the current quality of the experimental tensiometry. In our interpretation of the experiment, we will take care to minimize the errors in the values of the adsorption parameter via two routes: (i) whenever data for several homologues are available, we will fit them simultaneously, instead of fitting data for each surfactant separately (as in Ref. [5]); (ii) in any case in which we are able to give plausible value, even approximated to $5-10 \%$, of a parameter involved in our models, we will use this value instead of optimizing it. We will consider the most important parameter, the adsorption constant, separately in Sec. 3.1, where the parameters of our model of $K_{\mathrm{a}}$, Eqs. (13)-(14), are determined, largely from independent sources (data for solubility, partitioning, etc.). In Sec. 3.3-3.5, we demonstrate how the findings for $K_{\mathrm{a}}$, together with crystallographic \& collapse data for $\alpha$ and the theoretical results for $\beta$ can be used to predict the adsorption behaviour of many amphiphiles at $\mathrm{W} \mid \mathrm{O}$ and $\mathrm{W} \mid \mathrm{A}$ interfaces.

\subsection{Direct determination of the adsorption constant $K_{\mathrm{a}}$}

We will now summarize briefly the results from Ref. [8] for the adsorption constant determined via a polynomial fit with the virial expansion (6) over the initial region of the surface tension isotherm. This approach has the advantage of being model independent - that is why we call it direct approach. The method was used previously [8] to determine $K_{\mathrm{a}}$ of 50 non-ionic amphiphiles, which allowed us to draw the boundary line between the cohesive and non-cohesive surfactants. The findings presented here differ from those in Ref. [8] in several respects: some new data sources are used; the procedure has been improved; an error in the values of the partition coefficients has been corrected ${ }^{5}$. In this section, the data for strongly cohesive monolayers are disregarded for brevity (they will be treated in Sec. 3.5 with more 
advanced models). The details about the data handling are presented in the S3.

A typical regression with Eq. (6) is illustrated in Fig. 1 with decanol at W|O. The virial fit of the data involves two parameters - the adsorption constant $K_{\mathrm{a}}$ and the second virial coefficient $B_{2}$. However, since $B_{2}$ is obtained with rather high dispersion, only the values of $K_{\mathrm{a}}$ will be discussed. The results are presented in Fig. 3 as $\ln K_{\mathrm{a}}$ vs. $n$ for four homologous series: at $\mathrm{W} \mid \mathrm{O}$ - for fatty alcohols and acids, and at W|A - for N-n-alkyl-N,N-dimethylglycines $\left(\mathrm{C}_{n} \mathrm{H}_{2 n+1} \mathrm{Me}_{2} \mathrm{~N}^{+} \mathrm{CH}_{2} \mathrm{COO}^{-}\right)$and short chain length homologues of n-alkyldimethylphosphine oxides $\left(\mathrm{C}_{n} \mathrm{H}_{2 n+1} \mathrm{Me} 2 \mathrm{PO}\right)$. The $\mathrm{W} \mid \mathrm{O}$ data of Aveyard refer to oil-soluble alcohols [29]; we calculated the respective concentration of the alcohol in the aqueous solution (Fig. 1) by using the data for the partition coefficients ${ }^{5}$ [30] and the tetramerisation equilibrium of the alcohol in the alkane phase [31], as described in the S3. The tensiometric data for W|A are taken from various sources (cf. Table 1 for the complete list). Data for butanoic and pentanoic acids at water|benzene $(\mathrm{W} \mid \mathrm{B})$ at $35^{\circ} \mathrm{C}$ by Chatterjee and Chattoraj [69] is also processed (Table 1).

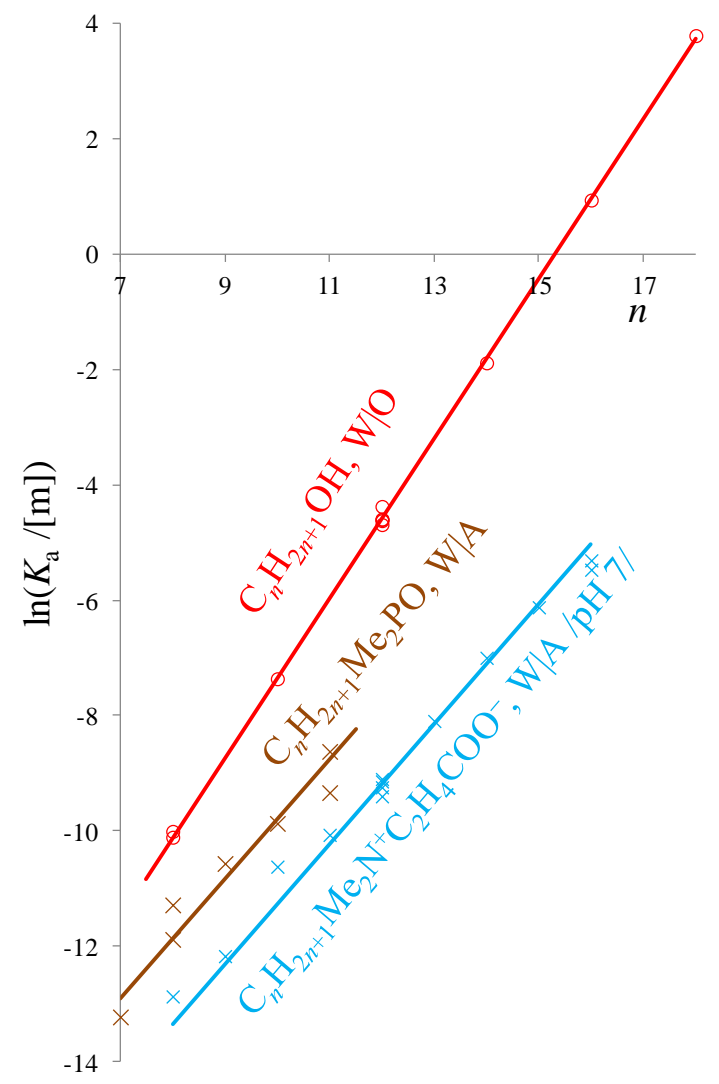

Fig. 3. Dependence of the logarithm of the adsorption constant $\ln K_{\mathrm{a}}$ on the chain length $n$ : results from fit of tensiometric data with the virial expansion of $\pi^{\mathrm{S}}(C)$, Eq. (6). The lines represent the theoretical $\ln K_{\mathrm{a}}(n)$ dependence (25) with fixed slopes: $\Delta \mu_{\mathrm{CH}_{2}} / k_{\mathrm{B}} T=1.39$ for $\mathrm{W} \mid \mathrm{O}$ and 1.04 for $\mathrm{W} \mid \mathrm{A}$ data; the values of the intercept $\ln K_{\mathrm{a} 0}$ and the sources for the tensiometric data used to obtain $K_{\mathrm{a}}$ are given in Table 1.

For the interpretation of the data in Fig. 3, we represent the theoretical expression (13)-(14) for $K_{\mathrm{a}}$ 
as:

$$
\ln K_{\mathrm{a}}=\ln K_{\mathrm{a} 0}+n \Delta \mu_{\mathrm{CH}_{2}} / k_{\mathrm{B}} T .
$$

Here, $\ln K_{\mathrm{a} 0}$ is the intercept of the lines in Fig. 3 - it is given by ${ }^{6}$ :

$$
\ln K_{\mathrm{a} 0}=\ln \delta_{\mathrm{a}}+\left(\Delta \mu_{\mathrm{CH}_{3}}-\Delta \mu_{\mathrm{CH}_{2}}+\alpha_{\perp} \sigma_{0}+\Delta \mu_{\text {head }}\right) / k_{\mathrm{B}} T,
$$

where $\delta_{\mathrm{a}}$ is given by Eq. (14). Eq. (25) is a form of Traube's rule [44].

Comparison of these equations with the $K_{\mathrm{a}}$ data requires the knowledge of the parameters in Eq. (26). The value of the length per methylene group $l_{\mathrm{CH}_{2}}=1.26 \AA$ follows from the geometry of the hydrocarbon chain [65]. We assume that the area $\alpha \perp$ of the interface disappearing during the adsorption of the surfactant molecule is close to the crystallographic area of the hydrocarbon chain. Crystallographic data for solid alkanes [70,71] and data for the area of collapse in Langmuir trough of insoluble alcohols [63,72] yield average area $\alpha_{\perp}$ of $18.2 \pm 0.4 \AA^{2}$. The interfacial tension $\sigma_{0}$ of the pure water|alkane interfaces in the experiments of Aveyard and Briscoe [29] was $53 \pm 0.5 \mathrm{mN} / \mathrm{m}$. For adsorption of noncohesive surfactants at the $\mathrm{W} \mid \mathrm{A}$ surface at $25^{\circ} \mathrm{C}$, the value $\sigma_{0}=72.2 \mathrm{mN} / \mathrm{m}$ must be used, and for water|benzene at $35^{\circ} \mathrm{C}, \sigma_{0}=32 \mathrm{mN} / \mathrm{m}$. Thus, the contribution of the term $-\alpha_{\perp} \sigma_{0}$ to the adsorption energy is of the order of $-2-3 \times k_{\mathrm{B}} T$, which is by no means negligible. The value of the first term in Eq. (26), standing for the adsorption thickness, at $\mathrm{W} \mid \mathrm{O}$ is $\ln \left(\delta_{\mathrm{a}} /[\mathrm{m}]\right)=-23.8$. Note that this is far smaller than the common assumption that $\delta_{\mathrm{a}} \approx n l_{\mathrm{CH}_{2}}$ [24], which for, e.g., decanol is about $13 \AA$, or $\ln \left(\delta_{\mathrm{a}} /[\mathrm{m}]\right)=-20.5$, by about +3 larger than the correct value.

The most important parameter in Eqs. (25)-(26) is $\Delta \mu_{\mathrm{CH}_{2}}$. Even a small error in the value of $\Delta \mu_{\mathrm{CH}_{2}}$ can have significant effect on the adsorption constant since $K_{\mathrm{a}}$ is proportional to $\exp \left(n \Delta \mu_{\mathrm{CH}_{2}} / k_{\mathrm{B}} T\right)$. The experimental value of $\Delta \mu_{\mathrm{CH}_{2}}$ vary within few percent from author to author [62,1,19,20,24,44,73]. We analysed a large number of experimental data for the adsorption constants of ionic [7] and non-ionic surfactants [8], partition coefficients (cf. Eq. (45)) and solubility data of homologous series of alkanes (considered in S4) and reached the conclusion that the best values are $\Delta \mu_{\mathrm{CH}_{2}} / k_{\mathrm{B}} T=1.39 \pm 0.03$ for transfer of $-\mathrm{CH}_{2}-$ from oil to water, and $\Delta \mu_{\mathrm{CH}_{2}} / k_{\mathrm{B}} T=1.04 \pm 0.06$ for transfer from air to water. The transfer energy $\Delta \mu_{\mathrm{CH}_{3}}$ for the terminal $-\mathrm{CH}_{3}$ group can also be estimated from the data [73] for solubility of alkanes in water - from the analysis in S4, we found the value $\Delta \mu_{\mathrm{CH}_{3}} / k_{\mathrm{B}} T=2.75 \pm 0.07$. This is close to the value 2.79 following from a geometrical model used previously by us [46], but disagrees with the often cited number $\Delta \mu_{\mathrm{CH}_{3}} / k_{\mathrm{B}} T=3.55[65]$. 
Table 1. Experimental intercepts $\ln \left(K_{\mathrm{a} 0} /[\mathrm{m}]\right)$ determined directly from the linear dependences $\ln K_{\mathrm{a}} \mathrm{vs.} n$ for different homologous series and $\ln \left(K_{\mathrm{a} 0} /[\mathrm{m}]\right)$ calculated from the fit with the SD model.

\begin{tabular}{|c|c|c|c|c|c|c|c|c|}
\hline \multirow[b]{2}{*}{ surfactant $^{\mathrm{a}}$} & \multirow[b]{2}{*}{$\begin{array}{c}\text { range } \\
\text { of } n\end{array}$} & \multirow[b]{2}{*}{$\begin{array}{l}\text { inter- } \\
\text { face }\end{array}$} & \multirow[b]{2}{*}{$\begin{array}{c}\sigma(C) \\
\text { data was } \\
\text { from } \\
\text { Refs. }\end{array}$} & \multirow[b]{2}{*}{$\frac{\Delta \mu_{\mathrm{CH}_{2}}}{k_{\mathrm{B}} T}$} & \multicolumn{2}{|c|}{ Directly calculated } & \multicolumn{2}{|c|}{ SD model ${ }^{\mathrm{b}}$} \\
\hline & & & & & $\ln \left(K_{\mathrm{a} 0} /[\mathrm{m}]\right)$ & $\frac{\Delta \mu_{\text {head }}{ }^{\mathrm{c}}}{k_{\mathrm{B}} T}$ & $\ln \left(K_{\mathrm{a} 0} /[\mathrm{m}]\right)$ & $\frac{\Delta \mu_{\text {head }}{ }^{\mathrm{c}}}{k_{\mathrm{B}} T}$ \\
\hline $\mathrm{C}_{n} \mathrm{H}_{2 n+1} \mathrm{OH}$ & $8 \div 18$ & $\mathrm{~W} \mid \mathrm{O}$ & 29 & 1.39 & $\begin{array}{c}-21.26 \\
\pm 0.09 \\
\end{array}$ & -1.2 & $-21.1^{\mathrm{d}}$ & -1.1 \\
\hline $\mathrm{C}_{n-1} \mathrm{H}_{2 n-1} \mathrm{COOH}$ & $4 \div 5$ & $\mathrm{~W} \mid \mathrm{B}$ & $69^{f}$ & 1.39 & $-22.4 \pm 0.1$ & $\sim 0^{\mathrm{g}}$ & $-22.3^{\mathrm{d}}$ & $+0.1^{\mathrm{g}}$ \\
\hline $\mathrm{C}_{n} \mathrm{H}_{2 n+1} \mathrm{Me}_{2} \mathrm{PO}$ & $7 \div 11$ & $\mathrm{~W} \mid \mathrm{A}$ & $2,81-83$ & 1.04 & $-20.2 \pm 0.4$ & -0.9 & $-20.0^{\mathrm{d}}$ & -0.7 \\
\hline $\mathrm{C}_{n} \mathrm{H}_{2 n+1} \mathrm{Me}_{2} \mathrm{~N}^{+} \mathrm{CH}_{2} \mathrm{COO}^{-}$ & $8 \div 16$ & $\mathrm{~W} \mid \mathrm{A}$ & $93,94,83^{\mathrm{e}}$ & 1.04 & $-21.7 \pm 0.15$ & -2.4 & $-21.6^{\mathrm{d}}$ & -2.3 \\
\hline
\end{tabular}

${ }^{a}$ Cf. S1 for the surfactants' names. ${ }^{b}$ Results from the fit over all data with Eqs. (10)-(12) of SD, cf. Sec. 3.3\&3.4 below. ${ }^{\mathrm{c}}$ Calculated using Eq. (27). ${ }^{\mathrm{d}}$ Results from Table 2. ${ }^{\mathrm{e}}$ Data for $\mathrm{pH}=7$ (the compound is in its zwitterionic form). ${ }^{\mathrm{f}}$ Only data for high adsorption is taken into account, where the dissociation degree is less than $3 \% .{ }^{\mathrm{g}}$ Calculated with the correction $-\Delta \mu_{\mathrm{CH}_{2}} / k_{\mathrm{B}} T$, according to footnote 6 .

The experimental value of the intercept $\ln K_{\mathrm{a} 0}$ was determined directly from the data for several members of a homologous series, Fig. 3. From the comparison of this value of $\ln K_{\mathrm{a} 0}$ with the theoretical expression (26), one can determine the value of the empirical correction $\Delta \mu_{\text {head: }}$

$$
\Delta \mu_{\text {head }} / k_{\mathrm{B}} T=\ln \left(K_{\mathrm{a} 0} / \delta_{\mathrm{a}}\right)-\left(\Delta \mu_{\mathrm{CH}_{3}}-\Delta \mu_{\mathrm{CH}_{2}}+\alpha_{\perp} \sigma_{0}\right) / k_{\mathrm{B}} T .
$$

The results for $\ln K_{\mathrm{a} 0}$ and the quantity $\Delta \mu_{\text {head }}$ for the four homologous series studied are presented in Table 1. For the purpose of comparison, the respective results obtained from the fit of the same tensiometric data with the sticky disc model of Ivanov et al. (cf. Sec. 3.3-3.5 for details) are also given. The following remarks can be made about these data [8].

(i) The slope of the experimental line $\ln K_{\mathrm{a}}$ vs. $n$ of surfactants at $\mathrm{W} \mid \mathrm{O}$ (alcohols in Fig. 3 and acids in Table 1) is $\Delta \mu_{\mathrm{CH}_{2}} / k_{\mathrm{B}} T=1.39$ whereas for surfactants at $\mathrm{W} \mid \mathrm{A}$ (glycines and short-chained phosphineoxides in Fig. 3) this slope is $\Delta \mu_{\mathrm{CH}_{2}} / k_{\mathrm{B}} T=1.04$, as expected.

(ii) For alcohols at $\mathrm{W} \mid \mathrm{O}, \Delta \mu_{\text {head }}$ is of the order of $-\Delta \mu_{\mathrm{CH}_{2}}$ (cf. Table 1), as if one methylene group remains immersed in the water phase rather than in the hydrophobic phase. This throws some light on the origin of the parameter $\Delta \mu_{\text {head }}$ in Eq. (26). It was suggested long ago that one methylene group adjacent to the hydrophilic-lyophilic centre behaves as a hydrophilic entity due to the influence of the adjacent polar group (Chap. 3 and Eq. 6-4 of Tanford [65]; see also [74,75]). Our result seems to agree with this hypothesis. The negative values (-0.9 and -2.4) obtained for $\Delta \mu_{\text {head }} / k_{\mathrm{B}} T$ of $\mathrm{C}_{n} \mathrm{H}_{2 n+1} \mathrm{Me}_{2} \mathrm{PO}$ and 
$\mathrm{C}_{n} \mathrm{H}_{2 n+1} \mathrm{Me}_{2} \mathrm{~N}^{+} \mathrm{CH}_{2} \mathrm{COO}^{-}$at $\mathrm{W} \mid \mathrm{A}$ also confirms this conclusion, as the two $-\mathrm{CH}_{3}$ groups attached to the $\mathrm{P}$ or $\mathrm{N}$ atoms do not contribute to the adsorption energy, i.e. they do not behave as hydrophobic entities as well. The more negative value $\Delta \mu_{\text {head }} / k_{\mathrm{B}} T=-2.4$ for glycines at $\mathrm{W} \mid \mathrm{A}$ is likely related to the large dipole moment of the $\mathrm{Me}_{2} \mathrm{~N}^{+} \mathrm{CH}_{2} \mathrm{COO}^{-}$group and the respective repulsive image force between the dipole and the surface. Finally, for acids at water|benzene we obtained $\Delta \mu_{\text {head }} \approx 0$. This is probably due to the relatively low polarity of the $>\mathrm{C}=\mathrm{O}$ group compared to $-\mathrm{OH}$ group: if the $>\mathrm{C}=\mathrm{O}$ group is not polar enough to polarize the adjacent $-\mathrm{CH}_{2}-$ group, no partial immersion of the hydrophobic chain should be expected.

Our model of the adsorption constant $K_{\mathrm{a}}$ is confirmed by the following findings: (i) the good linearity of the dependence $\ln K_{\mathrm{a}}$ vs. $n$ with the correct slopes $\Delta \mu_{\mathrm{CH}_{2}} / k_{\mathrm{B}} T=1.39$ for adsorption on $\mathrm{W} \mid \mathrm{O}$ and $\Delta \mu_{\mathrm{CH}_{2}} / k_{\mathrm{B}} T=1.04$ for adsorption on $\mathrm{W} \mid \mathrm{A}$. (ii) The coincidence of the experimental values of the intercept $\ln K_{\mathrm{a} 0}$ with the theoretical ones, Eq. (26), adjusted for the immersion of one methylene group into the water phase. (iii) A further proof is the temperature dependence of the adsorption constant in the LE phase of heptanol predicted by Eqs. (25)-(26) - it was shown in Ref. [8] that it agrees with the experimental data of Vochten and Petre [76]. (iv) Eqs. (25)-(26) were successfully used in Ref. [7] to interpret the effect of the nature of the oil phase on the adsorption constant of ionic surfactants. This gives us enough confidence to consider below the theoretical Eqs. (25)-(26) for $K_{\mathrm{a}}$ as proven.

\subsection{Treatment of the experimental data with a theoretical adsorption model}

We will now apply a more detailed approach to the tensiometric data considered in the previous section. First, data for the entire concentration range will be used. Second, the fit will be performed with the theoretical equations of state and adsorption isotherms presented in Sec. 2.1 in order to determine all basic adsorption parameters $\left(K_{\mathrm{a}}, \alpha \& \beta\right)$. We follow the regression procedure used by Danov et al. [5] which permits significant decrease of the number of free parameters involved. In addition, for most parameters we use independent experimental data or theoretical results. For the adsorption constant, the linear dependence of $\ln K_{\mathrm{a}}$ on $n$ and the value of its slope are known from the results of Sec. 3.1. The area $\alpha$ is expected to be independent of $n$ and it can be determined from the collapse area of insoluble monolayers. Finally, the value of $\beta$ at any $n$ can be calculated from Eq. (21).

In our approach, the regression with the theoretical surface pressure $\pi_{\mathrm{th}}^{\mathrm{S}}$ is over the data for an entire homologous series of surfactants. Therefore, the table of experimental values has three columns: $n$ and $C_{i}$ vs. $\pi_{n, i}^{\mathrm{S}}$, and it depends on a set of molecular parameters of the whole homologous series such as $K_{\mathrm{a} 0}$, 
$\Delta \mu_{\mathrm{CH}_{2}}, \alpha$ etc. that do not change with $n$. The merit function is therefore given by:

$$
\operatorname{dev}^{2}\left(K_{\mathrm{a} 0}, \Delta \mu_{\mathrm{CH}_{2}}, \alpha\right)=\frac{\sum\left[\pi_{n, i}^{\mathrm{s}}-\pi_{\mathrm{th}}^{\mathrm{s}}\left(C_{i}, n ; K_{\mathrm{a} 0}, \Delta \mu_{\mathrm{CH}_{2}}, \alpha\right)\right]^{2}}{N-1},
$$

where the total number of experimental points $N=\Sigma N_{n}$ for the whole homologous series is greatly increased compared to the ones of single homologue, $N_{n}$, which increases the reliability of the results.

\subsection{Water|oil interface}

The adsorption at $\mathrm{W} \mid \mathrm{O}$ interfaces is easier for analysis because it allows an important simplification, namely, using the assumption that the attractive parameter $\beta$ is small, even negligible [62]. This allows us to compare the various ways of interpreting the experimental data and to choose the most efficient procedure. We fit simultaneously all $N=129$ data points from Ref. [29] for alcohols of different carbon

numbers $n$ (unlike Sec. 3.1, where only data for dilute adsorption layers were used and every interfacial pressure isotherm was fitted separately).

Let us first consider the HFL model, which is appropriate for $\mathrm{W} \mid \mathrm{O}$ interface since $\beta \approx 0$. The merit function is given by Eq. (28), where $\pi_{\mathrm{th}}^{\mathrm{S}}\left(C_{i}, n ; K_{\mathrm{a} 0}, \Delta \mu_{\mathrm{CH}_{2}}, \alpha\right)$ is defined by Eqs. (1)\&(4) of HFL, together with Eq. (25) for the dependence of the adsorption constant $K_{\mathrm{a}}$ on $n$. Thus, instead of using 2 parameters $\left(K_{\mathrm{a}} \& \alpha\right)$ for every homologue, which makes 12 parameters altogether for the 6 alcohols studied by Aveyard and Briscoe, we use only 3 parameters $\left(K_{\mathrm{a} 0}, \Delta \mu_{\mathrm{CH}_{2}} \& \alpha\right)$ for the whole series. In addition, we know with good accuracy $[7,8]$ that $\Delta \mu_{\mathrm{CH}_{2}}=1.39 \times k_{\mathrm{B}} T$ (cf. Sec. 3.1). We can also determine the actual molecular area $\alpha$ from crystallographic data for solid alkanes [70,71] and from the area of collapse of insoluble monolayers of alcohols [63,72]: these data yield average area per molecule of $\alpha_{\perp}=18.2 \pm 0.4$ $\AA^{2}$. This value must be corrected by a packing factor: for hexagonal packing, $\alpha_{\perp}$ must be divided by 1.10 (which is the ratio between the area of a hexagon and the inscribed circle) to obtain the actual area $\alpha=$ $16.5 \pm 0.4 \AA^{2}$ of the hard disc $[8,77]$. In this way, a single parameter is left, $K_{\mathrm{a} 0}$, for the whole data set. 


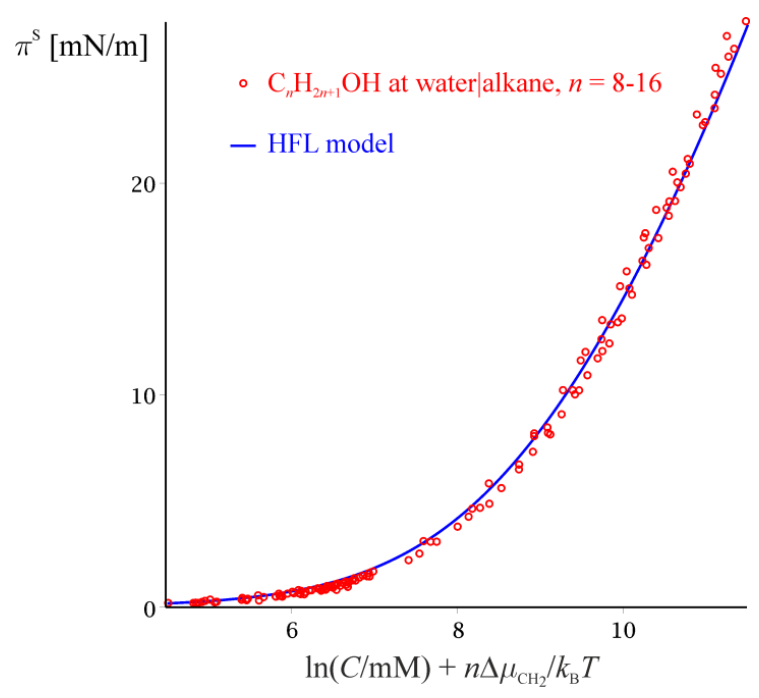

Fig. 4. Interfacial pressure $\pi^{\mathrm{S}}$ vs. logarithm of the scaled surfactant concentration, $\ln \left[C \times \exp \left(n \Delta \mu_{\mathrm{CH}_{2}} / k_{\mathrm{B}} T\right)\right]$. Data for oil-soluble fatty alcohols (chain length $n=8-18$ ) at $\mathrm{W} \mid \mathrm{O}$ interface, where the oil is normal alkane with chain length $n_{\mathrm{A}}=8-16 ; T=20^{\circ} \mathrm{C}$. $C$ is surfactant concentration in the water phase recalculated from the data of Aveyard and Briscoe [29] as described in S3. The data points for all $\mathrm{C}_{n} \mathrm{H}_{2 n+1} \mathrm{OH}$ homologues fall on a single curve, which confirms the scaling behaviour predicted by Eq. (29). The line is calculated with the HFL model, Eqs. (29)\&(1), with a single fitting parameter, $K_{\mathrm{a} 0}=7.97 \AA$.

The decrease of the number of free parameters from 12 to 1 can be illustrated in the following manner. We insert the expression (25) for $K_{\mathrm{a}}$ into HFL adsorption isotherm (4) to obtain:

$$
K_{\mathrm{a} 0} C \exp \frac{n \Delta \mu_{\mathrm{CH}_{2}}}{k_{\mathrm{B}} T}=\frac{\Gamma}{1-\alpha \Gamma} \exp \left[\frac{\alpha \Gamma(3-2 \alpha \Gamma)}{(1-\alpha \Gamma)^{2}}\right] .
$$

According to this equation, if one plots $\Gamma$ (or $\left.\pi^{S}\right)$ against $C \times \exp \left(n \Delta \mu_{\mathrm{CH}_{2}} / k_{\mathrm{B}} T\right)$, the resulting curves for all homologues must coincide since none of the remaining parameters is function of $n$. It is so indeed as shown in Fig. 4, where all data points fall on a single curve. The 1-parametric fit of the data with HFL model, Eqs. (29)\&(1), is also shown in Fig. 4; the value of the fitting parameter is $\ln \left(K_{\mathrm{a} 0} /[\mathrm{m}]\right)=-$ $20.95 \pm 0.1$.

Let us now compare this result to those obtained by alternative models - comparison is given in Table S3 in S6. Rows $\{1-2\}$ of Table S3 compare the fitting parameters found with two variants of the HFL regression: a 2-parametric fit with adjustable $K_{\mathrm{a} 0}$ and $\alpha$ and a 1-parametric fit with adjustable $K_{\mathrm{a} 0}$ ( $\alpha$ is fixed to $16.5 \AA^{2}$ ). The 2-parametric fit yields smaller standard deviation $d e v=0.40 \mathrm{mN} / \mathrm{m}$, but $\alpha$ is unreasonably small, $14.2 \AA^{2}$. On the other side, the use of a 1-parametric fit with fixed $\alpha=16.5 \AA^{2}$ increases the deviation to $d e v=0.49 \mathrm{mN} / \mathrm{m}$. The unreasonably low value of $\alpha$ in the former case and the larger deviation in the latter suggest that there is probably a problem with the HFL model. We blamed 
the neglected lateral attraction in HFL model for the problem. To check this hypothesis, we assumed that there is a lateral attraction leading to positive value of the attraction parameter $\beta$ at the $\mathrm{W} \mid \mathrm{O}$ interface. Besides, the fact that the points in Fig. 4 for all homologues lay on the same master curve suggests that the respective value of $\beta$ must be independent of the hydrophobic chain length $n$. We used fixed values for the transfer energy $\Delta \mu_{\mathrm{CH}_{2}}=1.39 \times k_{\mathrm{B}} T$ and the area $\alpha=16.5 \AA^{2}$ and two free parameters, $K_{\mathrm{a} 0}$ and $\beta$. We minimized the respective merit function $\operatorname{dev}^{2}\left(K_{\mathrm{a} 0}, \beta\right)=\Sigma\left[\pi_{n, i}^{\mathrm{S}}-\pi_{\mathrm{hh}}^{\mathrm{S}}\left(C_{i}, n ; K_{\mathrm{a} 0}, \beta\right)\right]^{2} /(N-1)$, where the theoretical surface pressure $\pi_{\mathrm{th}}^{\mathrm{S}}$ is the one following from the SD model, Eqs. (10)-(12). The deviation obtained with this regression model is $0.4 \mathrm{mN} / \mathrm{m}$ (the same as that for the 2-parametric model of HFL, cf. rows $\{2 \& 5\}$ in Table S3). Since $\beta$ is independent of $n$, the obtained value $\beta=0.332$ probably refers to the attractive hydrophobic interaction [65,78] only between the $-\mathrm{CH}_{2}-$ groups adjacent to the polar head group, which remain immersed in the water phase (cf. comment (ii) below Eq. (27)).

The results from the fit of the tensiometric data for alcohols at $\mathrm{W} \mid \mathrm{O}$ with the models of Volmer and Langmuir (no attraction, Eqs. (5)), are shown in rows $\{3-4\}$ in Table S3. The 2-parametric fits with these models yield practically the same mean deviation and the same value of $K_{\mathrm{a} 0}$ as HFL. Therefore, the only parameter which can be used to distinguish between the three models is the actual area $\alpha$. With Volmer's model it is $\alpha_{\mathrm{V}}=24.4 \AA^{2}$. This value is too high - about $50 \%$ larger than $16.5 \AA^{2}$ following from collapse/crystallographic area of the molecule. If the relation (18) between $\alpha_{\mathrm{V}}$ and the true geometrical area $\alpha$ is valid, then $\alpha=\alpha_{\mathrm{V}} / 2=12.2 \AA^{2}$, which is again unrealistic (it is $25 \%$ smaller than the expected value $16.5 \AA^{2}$ ). Therefore, even though the mean deviation of Volmer's model is in fact slightly smaller than the one of HFL model, the accuracy of this model is dubious. The situation is worse with Langmuir's model, giving $\alpha_{\mathrm{L}}=39 \AA^{2}$ (or $\alpha=9.8 \AA^{2}$ according to Eq. (18)).

We can instead use van der Waals and Frumkin models, Eqs. (7), with $\beta_{\mathrm{L}}=-1.5$ and $\beta_{\mathrm{V}}=-1$. As discussed in Sec. 2.3, these values correspond to the absence of attraction (i.e. to $\beta=0$, cf. Eq. (19)) so in principle, with these particular values of $\beta_{\mathrm{L}}$ and $\beta_{\mathrm{V}}$, van der Waals and Frumkin are better models of a hard-disc 2-D fluid than Volmer's and Langmuir's. The 2-parametric fits with these EOS yield the same deviation, $0.4 \mathrm{mN} / \mathrm{m}$, as all other 2-parametric models, rows $\{6-7\}$ in Table S3. The obtained values $\alpha_{\mathrm{V}}=18.2 \AA^{2}$ and $\alpha_{\mathrm{L}}=21.0 \AA^{2}$ are indeed close to the actual area $\alpha=16.5 \AA^{2}$, which proves the validity of the conditions (19). Yet, one must not forget that Eqs. (19) are merely a way to approximate a HFL-based EOS with Frumkin or van der Waals formulas (7), and it is obviously better to use directly HFL model instead. 
Table 2. Adsorption parameters of various homologous series of surfactants at $\mathrm{W} \mid \mathrm{O}$ and $\mathrm{W} \mid \mathrm{A}$ interfaces, obtained by the minimization of a merit function of the type of Eq. (28) using the sticky disc model (10)-(12).

\begin{tabular}{|c|c|c|c|c|c|}
\hline homologous series & interface & $\ln \left(K_{\mathrm{a} 0} /[\mathrm{m}]\right)^{\mathrm{i}}$ & $\alpha\left[\AA^{2}\right]$ & $\beta$ & $\operatorname{dev}[\mathrm{mN} / \mathrm{m}]$ \\
\hline $\mathrm{C}_{n} \mathrm{H}_{2 n+1} \mathrm{OH}^{\mathrm{a}}$ & water|alkane & -21.1 & $16.5^{\mathrm{j}}$ & 0.332 & 0.40 \\
\hline $\mathrm{C}_{n-1} \mathrm{H}_{2 n-1} \mathrm{COOH}^{\mathrm{b}}$ & water|benzene & -22.3 & $\overline{18.0^{\mathrm{j}}}$ & 1.60 & 0.4 \\
\hline $\mathrm{C}_{13} \mathrm{H}_{27} \mathrm{Me}_{2} \mathrm{PO}{ }^{\mathrm{c}}$ & water|hexane & -22.5 & $28.2^{\mathrm{k}}$ & $\approx 0$ & 1.1 \\
\hline $\mathrm{C}_{n} \mathrm{H}_{2 n+1} \mathrm{OH}(\text { non-coh. })^{\mathrm{d}}$ & \multirow{7}{*}{$\mathrm{W} \mid \mathrm{A}$} & -20.1 & \multirow{2}{*}{$16.5^{\mathrm{j}}$} & \multirow{2}{*}{ Eq. $(21)^{1}$} & 0.88 \\
\hline $\mathrm{C}_{n} \mathrm{H}_{2 n+1} \mathrm{OH}(\text { all })^{\mathrm{e}}$ & & -20.4 & & & 1.44 \\
\hline $\mathrm{C}_{n-1} \mathrm{H}_{2 n-1} \mathrm{COOH}(\text { non-coh. })^{\mathrm{d}}$ & & -20.4 & \multirow{2}{*}{$\underline{18}^{\mathrm{j}}$} & \multirow{2}{*}{$\underline{\text { Eq. }(21)^{1}}$} & 0.57 \\
\hline $\mathrm{C}_{n-1} \mathrm{H}_{2 n-1} \mathrm{COOH}(\text { all })^{\mathrm{e}}$ & & -20.2 & & & 0.93 \\
\hline $\mathrm{C}_{n} \mathrm{H}_{2 n+1} \mathrm{Me}_{2} \mathrm{PO}(\text { non-coh })^{\mathrm{f}}$ & & -20.0 & 28.2 & \multirow{2}{*}{ Eq. $(21)^{1}$} & 0.98 \\
\hline $\mathrm{C}_{n} \mathrm{H}_{2 n+1} \mathrm{Me}_{2} \mathrm{PO}(\text { all })^{\mathrm{g}}$ & & -19.7 & $29^{j}$ & & 2.0 \\
\hline $\mathrm{C}_{n} \mathrm{H}_{2 n+1} \mathrm{Me}_{2} \mathrm{~N}^{+} \mathrm{CH}_{2} \mathrm{COO}^{-}(\text {all })^{\mathrm{h}}$ & & -21.6 & 30.2 & 2.31 & 1.1 \\
\hline
\end{tabular}

${ }^{\text {a }}$ Alcohols are with chain length $n=8 \div 18$ (even $n$ only), oil phase is alkane with $n_{\mathrm{A}}=8 \div 16 ; T=20^{\circ} \mathrm{C}$. ${ }^{\mathrm{b}}$ Butanoic and pentanoic acid; $T=35^{\circ} \mathrm{C}$ (cf. S8). ${ }^{\mathrm{c}}$ Data for single homologue $(n=13)$ are available; $T=20^{\circ} \mathrm{C}(\mathrm{cf}$. S8). ${ }^{\mathrm{d}}$ Only data for the non-cohesive homologues $(n=3 \div 4)$ are considered, average $T=21^{\circ} \mathrm{C}$. ${ }^{\mathrm{e}}$ The data for all homologues $(n=3 \div 10)$ are considered, average $T=21{ }^{\circ} \mathrm{C} .{ }^{\mathrm{f}} n=7 \div 11$, average $T=23.5^{\circ} \mathrm{C} .{ }^{\mathrm{g}} n=7 \div 16, T=$ $23.5^{\circ} \mathrm{C}$. ${ }^{\mathrm{h}} n=8 \div 16$, average $T=20^{\circ} \mathrm{C}$. ${ }^{\mathrm{i}}$ The transfer energy in the expression (25) for the adsorption constant is fixed to $\Delta \mu_{\mathrm{CH}_{2}}=1.39 \times k_{\mathrm{B}} T$ for $\mathrm{W} \mid \mathrm{O}$ data and to $\Delta \mu_{\mathrm{CH}_{2}}=1.04 \times k_{\mathrm{B}} T$ for $\mathrm{W} \mid \mathrm{A}$ data. ${ }^{j}$ Value of the area, calculated

from data for the crystallographic and collapse area of alcohols and acids. ${ }^{\mathrm{k}}$ The value is fixed to the one obtained by fit of the tensiometric data for non-cohesive phosphineoxides at $\mathrm{W} \mid \mathrm{A}$. ${ }^{1}$ Fixed to the value predicted by the nonlinear Eq. (21). Note that the expression (21) for $\beta$ involves $\alpha$ as a parameter; this was accounted for in the optimization procedure.

Some additional analysis is carried out in S7, which shows that any optimization involving both $\alpha$ and $\beta$ as free parameters is unable to give their values with satisfying accuracy. Therefore, we consider the results from the 2-parametric SD model with free $K_{\mathrm{a} 0}$ and $\beta$ as more reliable than, e.g., a 3-parametric SD model with free $K_{\mathrm{a} 0}, \alpha$ and $\beta$.

We verified the obtained results for $\mathrm{C}_{n} \mathrm{H}_{2 n+1} \mathrm{OH}$ also by analysing in $\mathrm{S} 8$ the interfacial tension data from Ref. [79] for a water-soluble alcohol (butanol) adsorbed at water|dodecane interface. The available data for acids and tridecyldimethylphosphineoxide at the water|oil interface [80] have also been analysed with the 2-parametric SD model in S8. The area parameter $\alpha$ was fixed in both cases. To calculate $\alpha$ of 
acids, we used the data for the collapse area of several insoluble long-chained homologues $[63,20,19,43]$. The average value of these data is $19.8 \pm 0.8 \AA^{2}$, which is close to the crystallographic area $20.5 \AA^{2}$ quoted by Langmuir [20]. Using the correction factor for close packing 1.10, we find for the actual area $\alpha=$ $18.0 \pm 0.8 \AA^{2}$. For $\mathrm{C}_{13} \mathrm{H}_{27} \mathrm{Me}_{2} \mathrm{PO}$, we used the result $\alpha=28.2 \AA^{2}$ obtained from the fit of the data for the non-cohesive homologues at W|A (cf. Sec. 3.4 below). Two parameters, $K_{\mathrm{a} 0}$ and $\beta$, of the two surfactant series are determined from the regression, cf. Table 2 . The attraction parameter of $\mathrm{C}_{13} \mathrm{H}_{27} \mathrm{Me}_{2} \mathrm{PO}$ is close to zero, which is perhaps due to the larger area per molecule of this surfactant (the distance between the interacting neighbours is larger compared to the alcohols). For acids, we obtained $\beta=1.6$; this value may be erroneous, since the data for the two acids refer to concentrated aqueous solutions ( $200 \mathrm{mM}$ ), where the bulk activity coefficient probably starts to play a role - we neglected this effect due to the absence of data. The regressions are illustrated in S8.

Our overall conclusion is that the EOS of Frumkin and van der Waals might be useful for qualitative analysis when comparing the values of the adsorption parameters of several surfactants (e.g., relative values of the actual areas $\alpha$ or linear dependence of $\ln K_{\mathrm{a}}$ on $n$ ), but the absolute values of $\alpha$ and $\beta$ might be wrong - this may lead to erroneous physical conclusions, such as repulsion instead of attraction.

\subsection{Non-cohesive surfactants at the water|air surface}

We turn now to the adsorption at $\mathrm{W} \mid \mathrm{A}$ of non-cohesive non-ionic surfactants. Three homologous series have been analysed: the zwitterionic $\mathrm{C}_{n} \mathrm{H}_{2 n+1} \mathrm{Me}_{2} \mathrm{P}^{+} \mathrm{O}^{-}$, alcohols $\mathrm{C}_{n} \mathrm{H}_{2 n+1} \mathrm{OH}$ and acids $\mathrm{C}_{n-1} \mathrm{H}_{2 n-1} \mathrm{COOH}$ (at conditions where less than $2-3 \%$ of the adsorbed acid is dissociated). For the $\mathrm{C}_{n} \mathrm{H}_{2 n+1} \mathrm{Me}_{2} \mathrm{PO}$ homologues, we used tensiometric data from Refs. [2,81-83]. Collapse areas are available for insoluble long-chained $\mathrm{C}_{n} \mathrm{H}_{2 n+1} \mathrm{Me}_{2} \mathrm{PO}$ [84,2]: the average value which we calculated from these data is $31.9 \pm 0.5 \AA^{2}$. With the correction factor 1.10 for the close packing, it leads to $\alpha=29.0 \AA^{2}$. Based on the criteria summarized in Sec. 1.1, homologues with $n \leq 11$ behave non-cohesively, and those with $n \geq 12$ behave cohesively (undecyldimethylphosphineoxide is at the boundary of both behaviours). In this section, only the non-cohesive ones will be analysed.

$\mathrm{W} \mid$ A surface tension data for all alcohol homologues from propanol to decanol are available [1,50$53,76,83,85-87]$, at average temperature of $21 \pm 1^{\circ} \mathrm{C}$. They point out at cohesive behaviour, except for $n$ $=3-4-$ therefore, in this section, only the propanol and butanol are considered. The tensiometric data for $n=3-4$ are for quite concentrated solutions, since these molecules are not very surface active. Therefore, the ideal solution approximation is invalid for them. We calculated the activity of propanol 
in Addison experiments [85] using the data for the activity coefficient $\gamma_{\mathrm{C}_{3} \mathrm{H}_{7} \mathrm{OH}}$ from Ref. [88], which we interpolated with the formula $\ln \gamma_{\mathrm{C}_{3} \mathrm{H}_{7} \mathrm{OH}}=-5.48 \cdot v_{\mathrm{w}} C+9.20 \cdot\left(v_{\mathrm{w}} C\right)^{2}$, where $v_{\mathrm{w}}$ is the molar volume of water. The tensiometric data for very concentrated solutions of butanol were disregarded, since we found no reliable information for their activity coefficients. The area per molecule is assumed to be $16.5 \AA^{2}$, as in Sec. 3.3.

The data for carboxylic acids at low $\mathrm{pH}$ and average temperature $21 \pm 1^{\circ} \mathrm{C}[17,86,89-92]$ were dealt with similarly. In fact, the tensiometric curves of alcohols and acids of the same carbon number $n$ lay close to each other. Only the propanoic and butanoic acids behave non-cohesively and were considered in the current section. The hard-disc area is set to $18.0 \AA^{2}$, cf. Sec. 3.3.

Various models and procedures (based on those described in Sec. 3.2) have been used to fit the whole set of data for all homologues in a given homologous series simultaneously. For all surfactants at $\mathrm{W} \mid \mathrm{A}$, we used fixed values of the transfer energy $\Delta \mu_{\mathrm{CH}_{2}}=1.04 \times k_{\mathrm{B}} T$, as determined in Sec. 3.1 and Refs. $[8,7]$. Representative results are given in Table S4 in S6. The tensiometric data from various authors differ by about 1-3 $\mathrm{mN} / \mathrm{m}$, which is the reason for the relatively large mean deviations (compared to those for alcohols at $\mathrm{W} \mid \mathrm{O}$ in Table S3). The most important results from the fits of these data are discussed in the following paragraphs.

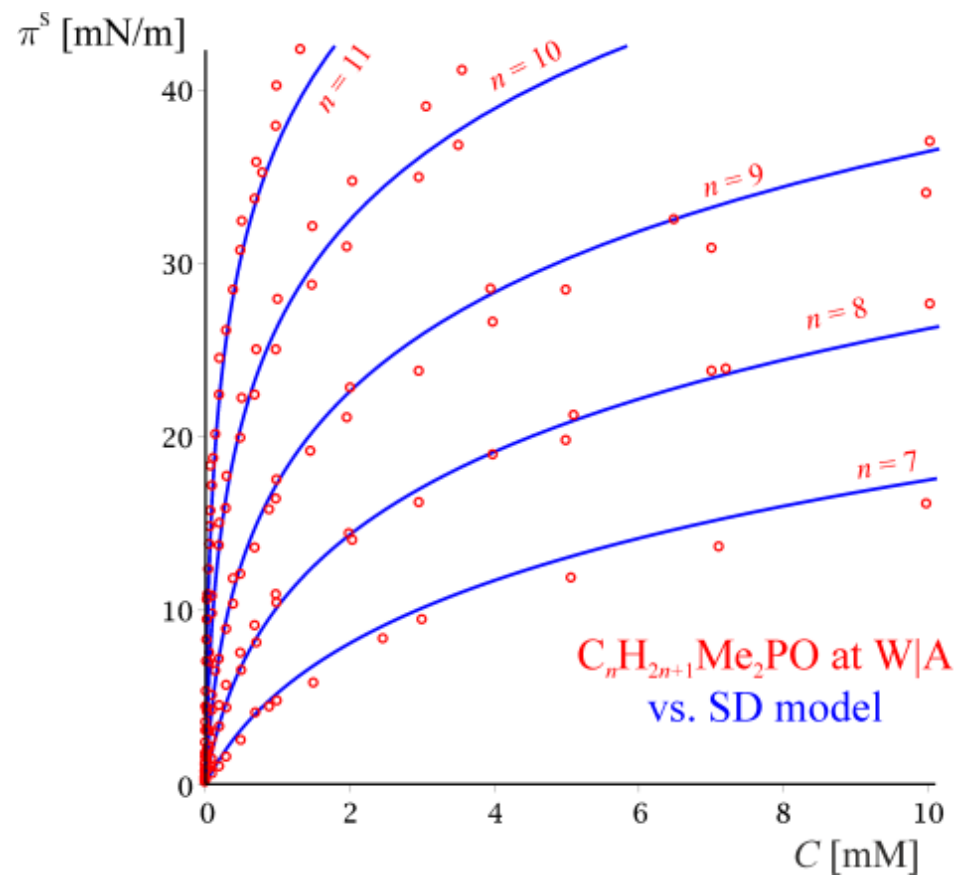

Fig. 5. Surface pressure $\pi^{\mathrm{S}}$ vs. concentration $C$ of the non-cohesive homologues of $\mathrm{C}_{n} \mathrm{H}_{2 n+1} \mathrm{Me}_{2} \mathrm{PO}$ at W|A.

Comparison with the continual SD model with two fitting parameters $\left(K_{\mathrm{a} 0}=21.2 \AA\right.$ and $\left.\alpha=28.2 \AA^{2}\right)$.

(i) The SD model for the non-cohesive $\mathrm{C}_{n} \mathrm{H}_{2 n+1} \mathrm{Me} 2 \mathrm{PO}$. In the case of surfactants at W|A the 
hydrocarbon chain attraction increase with $n$, and $\beta$ must be calculated via Eq. (21). Eqs. (10)-(12) of the SD theory, along with the theoretical expressions Eq. (21) for $\beta$ and Eq. (25) for $K_{\text {a define a model }}$ with only two free parameters: $K_{\mathrm{a} 0}$ and $\alpha$. The fits were performed over all data for all homologues simultaneously (a 3-dimensional mathematical surface $\pi^{\mathrm{S}}(C, n)$ ), as explained in Sec. 3.2. The results are visualized in Fig. 5, where the theoretically obtained curves $\pi^{\mathrm{S}}$ vs. $C$ for each $n$ are compared with the experimental data. The determined value of the actual area of $\mathrm{C}_{n} \mathrm{H}_{2 n+1} \mathrm{Me}_{2} \mathrm{PO}$ is $\alpha=28.2 \AA^{2}$. It differs from the one calculated above from the collapse or crystallographic areas, $29 \AA^{2}$, by less than $3 \%$. Note that the values of $\beta$ in this model are very sensitive to the values of $\alpha$ because of the strong dependence of $\beta$ on $\alpha$, cf. Eq. (21) and Fig. 2 - nonetheless, the deviation of the model from the experimental data is small (row $\{2\}$ in Table S4), which makes the obtained result for $\alpha$ even more trustworthy. We further made the model 1-parametric by setting the value of $\alpha$ equal to the one determined from the collapse areas. The deviation from the experimental data is nearly the same, rows $\{1-2\}$ in Table S4. The determined value of the intercept $\ln \left(K_{\mathrm{a} 0} /[\mathrm{m}]\right)$ is $-19.9 /-20.0$, close to -20.2 obtained in Sec. 3.1 directly by using virial expansion for the initial region of the surface tension isotherm (cf. Table 1). The values of $\beta$ will be discussed in Sec. 3.5 below.

We tried to fit the $\mathrm{C}_{n} \mathrm{H}_{2 n+1} \mathrm{Me}_{2} \mathrm{PO}$ data by using the SD model together with the linear dependence $\beta=\beta_{1}(n+1)$, Eq. (23), with $\beta_{1}=0.49$ as suggested by Smith [63], instead of the integral (21). The result is shown in row $\{3\}$ in Table $\mathrm{S} 4$ - the obtained deviation is too high. Therefore, one concludes that SD model is compatible only with the non-linear formula (21).

(ii) SIAL model for the non-cohesive $\mathrm{C}_{n} \mathrm{H}_{2 n+1} \mathrm{Me}_{2} \mathrm{PO}$. We tried to use the SIAL model with the theoretical $\beta$ from Eq. (21). It led, however, to too high deviations from the tensiometric data (standard deviation $1.6 \mathrm{mN} / \mathrm{m}$ if fixed value $\alpha=29 \AA^{2}$ is used). Instead, we then attempted Smith's formula (23) for $\beta(n)$, with the fixed value proposed by him [63], $\beta_{1}=0.49$ (row $\{5\}$ in Table S4). This leaves only one free parameter, $K_{\mathrm{a} 0}$, to be determined from the whole set of data for the phosphineoxide homologues. The deviation is still too high $(1.7 \mathrm{mN} / \mathrm{m})$. We carried out also 3-parametric fits with SIAL model, $\beta$ being given by Eq. (24), $\beta(n)=\beta_{0}+\beta_{1} n$. The results are $\beta_{0}=-0.08, \beta_{1}=0.346$ and $\ln K_{\mathrm{a} 0}=-20.5$ (row $\{6\}$ in Table S4). The deviation is comparable to the one of the SD model (although the latter model is 2 parametric, rows $\{2 \& 6\}$ in Table $S 4)$.

(iii) Van der Waals and Frumkin models for the non-cohesive $\mathrm{C}_{n} \mathrm{H}_{2 n+1} \mathrm{Me} 2 \mathrm{PO}$. Previous analysis of the models of van der Waals [5] and Frumkin [1,5] showed that the attraction parameter $\beta$ depends linearly on $n$ according to Eq. (23), similarly to what we found with SIAL model. This linear dependence 
contradicts the integral formula (21). The 4-parametric models of van der Waals and Frumkin (with parameters $\left.K_{\mathrm{a} 0}, \alpha, \beta_{0}, \beta_{1}\right)$ yields $\ln \left(K_{\mathrm{a} 0} /[\mathrm{m}]\right)=-19.9$ and -19.8 respectively (rows $\{8-9\}$ in Table S4), in agreement with the results from the SD and SIAL. The obtained area parameters are, however, too high: $\alpha_{\mathrm{V}}=33.0$ and $\alpha_{\mathrm{L}}=43.4 \AA^{2}$ for the two models, which is respectively by $14 \%$ and $50 \%$ larger than the value following from the areas determined from the collapse of insoluble monolayers. The values obtained for $\beta_{1}$ (0.273 and 0.206 for vdW and Frumkin) are small compared to the value 0.49 proposed by Smith.

(iv) The data for the non-cohesive $\mathrm{C}_{n} \mathrm{H}_{2 n+1} \mathrm{OH}$ and $\mathrm{C}_{n-1} \mathrm{H}_{2 n-1} \mathrm{COOH}$ were fitted with the SD model, where Eqs. (21) and (25) were assumed valid for $\beta$ and $K_{\mathrm{a}}$ respectively. We also fixed the value of the area per molecule to the one following from crystallographic and collapse data. This leaves a single parameter in the optimization procedure: $\ln K_{\mathrm{a} 0}$. Its value is $-20.1 \pm 0.1$ for alcohols and $-20.4 \pm 0.1$ for acids, Table 2. The value for acids so found can be compared with the theoretical one following from Eq. (26), namely, $\ln \left(K_{\mathrm{a} 0} /[\mathrm{m}]\right)=-20.3+\Delta \mu_{\text {head }} / k_{\mathrm{B}} T$ (cf. also footnote 5). The comparison reveals that the term $\Delta \mu_{\text {head }}=-0.1 \times k_{\mathrm{B}} T$ is almost negligible, i.e. there is no immersion of the hydrophobic chain. This confirms what we previously found with acids at $\mathrm{W} \mid$ benzene in Table 1. On the other hand, for alcohols $\Delta \mu_{\text {head }}=-0.8 \times k_{\mathrm{B}} T$, which suggests that the $-\mathrm{CH}_{2}$ - group next to the polar group remains immersed, as with the alcohols at $\mathrm{W} \mid \mathrm{O}$ in Table 1.

As a summary of this section, we again confirm the unreliability of the results from Frumkin and van der Waals models not only for $\mathrm{W} \mid \mathrm{O}$, but this time for $\mathrm{W} \mid \mathrm{A}$ surface as well. These models yield values of the adsorption constant concordant with those obtained with the direct approach in Sec. 3.1; however, the values of the areas $\alpha_{\mathrm{V}, \mathrm{L}}$ are too high, and the attraction parameters $\beta_{\mathrm{V}, \mathrm{L}}$ show no clear correlation to molecular characteristics. SIAL model leads to the expected crystallographic/collapse values of $\alpha$, but is incompatible with the non-linear formula $(21)$ for $\beta(n)$. It agrees with the experiment only if the linear dependence (24) for $\beta(n)$ is used instead, but then the respective parameters $\beta_{0}$ and $\beta_{1}$ again cannot be related to the molecular characteristics. Only the SD model exhibits no obvious flaws: it agrees well with the tensiometric data, it leads to the expected values of $\alpha$, and it agrees also with the predicted dependence (21) for $\beta(n)$.

\subsection{Continual approach for cohesive surfactants}


The data for both non-cohesive and cohesive soluble surfactants are commonly interpreted in the literature by using the continual models from Sec. 2.1.2 which is not always justified. That is why in Sec. 3.4 we used only data for the non-cohesive acids, alcohols and phosphineoxides. Now we will deal with the whole series, including both short-chained non-cohesive and long-chained cohesive homologues (using the same set of known parameters, $\Delta \mu_{\mathrm{CH}_{2}}, \alpha$ and $\beta$, as in Sec. 3.4.). However, the results from this section must be considered with caution. The result we will obtain now will be used in a subsequent publication to demonstrate that the partial approach has clear advantages over the continual one with strongly cohesive surfactants.

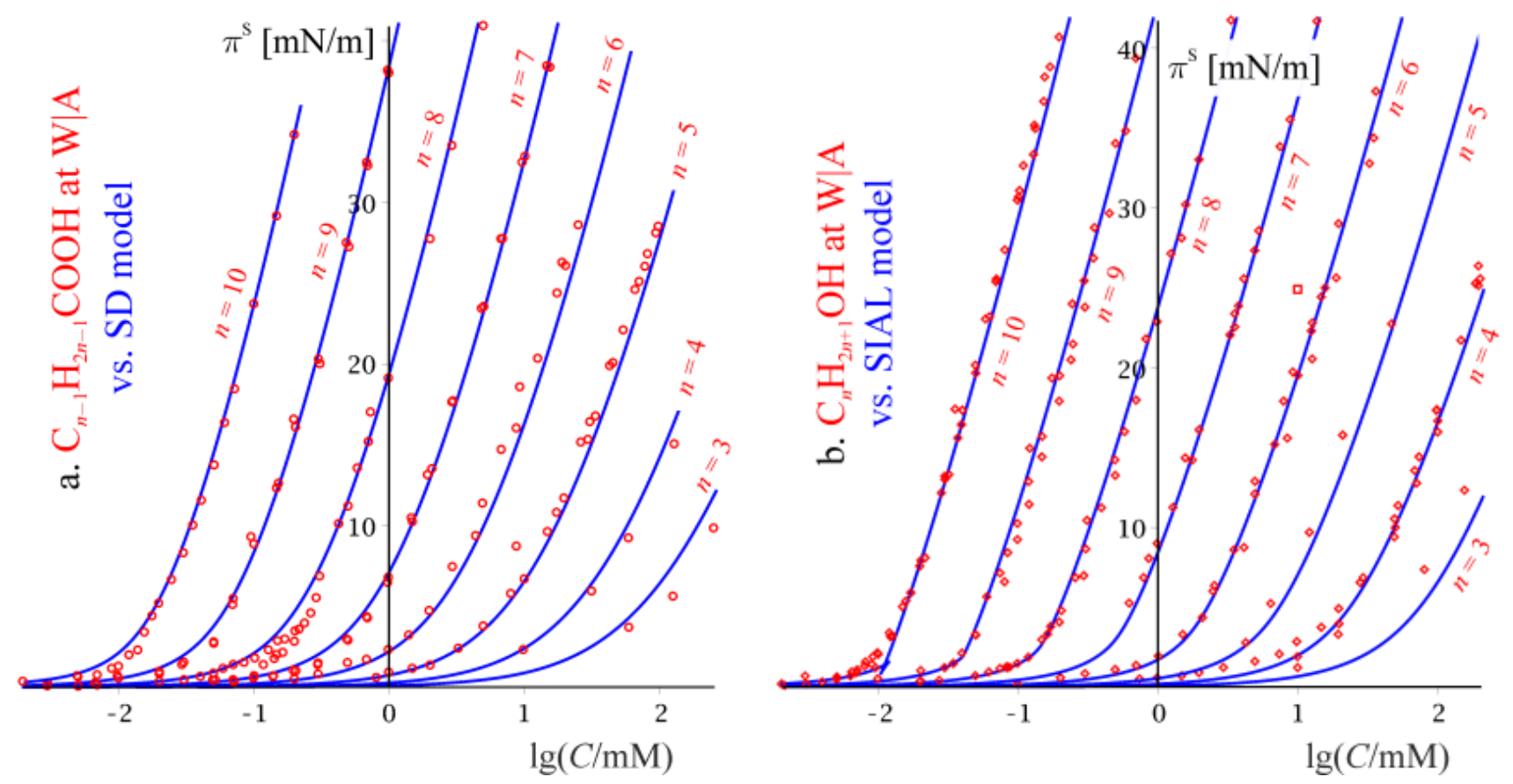

Fig. 6. Surface pressure $\pi^{\mathrm{S}}$ vs. decimal logarithm of concentration of homologous series of non-ionic surfactants at W|A. a. $\mathrm{C}_{n-1} \mathrm{H}_{2 n-1} \mathrm{COOH}$ - fit with the sticky disc model, Eqs. (10)-(12), with a single fitting parameter: $K_{\mathrm{a} 0}=$

$16.7 \AA$ (we take $\alpha=18 \AA^{2}$ and values of $\beta(n)$ calculated from Eq. (21)). b. $\mathrm{C}_{n} \mathrm{H}_{2 n+1} \mathrm{OH}$ - fit with the SIAL model, Eqs. (8)-(9), with 3 fitting parameters: $K_{\mathrm{a} 0}=11 \AA$ and $\beta=0.86+0.56 n$. The data refers to both cohesive $(n=5-10)$ and non-cohesive $(n=3-4)$ adsorption layers.

(i) The SD model, Eqs. (10)-(12), along with the theoretical expression Eq. (21) for $\beta$, were used with a single free parameter, $K_{\mathrm{a} 0}$ (Table 2). Not surprisingly, the obtained value of the fitting parameter $K_{\mathrm{a} 0}$ is unchanged compared to the one following from non-cohesive homologues only. The results for the acids are visualized in Fig. 6a, where the theoretically obtained curves $\pi^{\mathrm{S}}$ vs. $\lg C$ for each $n$ are compared with the experimental data. One sees that the SD continual model works relatively well even for cohesive surfactants. It fails only with the higher homologues near the kink between the gaseous and LE phases (cf. the acids with $n>7$ in Fig. 6a). This could be expected since the SD model does not 
predict phase transitions, whereas the highest homologues of the acids and the alcohols are rather close to the critical point of the phase transition gaseous-LE, cf. Sec. 3.6 below. The high deviation for alcohols (Table 2) is due to the same problem. The deviation from SD model obtained for all phosphineoxides (cohesive+non-cohesive) is worse than the deviation for the non-cohesive homologues alone (compare rows $\{1 \& 4\}$ of Table S4). This high deviation is, in fact, due only to the two highest homologues, which do not follow SD model at all.

We tested the SD model also with two adjustable parameters, by leaving free the actual area $\alpha$ and $K_{\mathrm{a} 0}$ (rows $\{15,22 \& 2\}$ of Table $\mathrm{S} 4$ ). The obtained $\alpha$ values for $\mathrm{C}_{n-1} \mathrm{H}_{2 n-1} \mathrm{COOH}, \mathrm{C}_{n} \mathrm{H}_{2 n+1} \mathrm{OH}$ and $\mathrm{C}_{n} \mathrm{H}_{2 n+1} \mathrm{Me}_{2} \mathrm{PO}$ at $\mathrm{W} \mid \mathrm{A}$ (only non-cohesive homologues for the latter) were 18.1, 17.3 and $28.2 \AA^{2}$ respectively. They differ by less than $6 \%$ from the ones calculated from the collapse or crystallographic areas $\left(18,16.5\right.$ and $29 \AA^{2}$ - the same values which worked for $\mathrm{W} \mid \mathrm{O}$ as well, Sec. 3.3).

The attraction parameters $\beta$ for all homologues of the acids and alcohols were calculated from Eq. (21) using the value of $\alpha$ obtained from the crystallographic area - their values are given in Table 3. For $\mathrm{C}_{n} \mathrm{H}_{2 n+1} \mathrm{Me}_{2} \mathrm{PO}$, we found that the area $\alpha=28.2 \AA^{2}$, obtained from the 2-parametric fit of the noncohesive homologues leads to better results than the value $29 \AA^{2}$ that follows from collapse areas. An important feature of the values of $\beta$ in Table 3 is the correlation between the cohesive/non-cohesive behaviour and the Boyle point of the homologous series. The Boyle point is the boundary at which attraction starts to prevail over repulsion. This corresponds to $B_{2}=0$, or from Eq. (15), to $\beta=2$; when $\beta$ $>2$, the $\pi^{\mathrm{S}}(C)$ dependence following from Eqs. (10)-(12) has a characteristic inflection. Judging from the $\beta$-values in Table 3, $\mathrm{C}_{5} \mathrm{H}_{11} \mathrm{COOH}, \mathrm{C}_{4} \mathrm{H}_{9} \mathrm{OH}$ and $\mathrm{C}_{12} \mathrm{H}_{25} \mathrm{Me}_{2} \mathrm{PO}$ are very close to their Boyle points. All shorter homologues are non-cohesive (above their Boyle temperature). All longer homologues are cohesive (below their Boyle temperature). This is an additional criterion for cohesive or non-cohesive behaviour of the adsorption layer besides those formulated in Sec. 1.1 and Ref. [8].

(ii) SIAL model. As already observed with the non-cohesive phosphineoxides, the SIAL model is incompatible with the theoretical non-linear $\beta(n)$ dependence from Eq. (21). Hence, we used SIAL in combination with Smith's formula (23) for $\beta$, with the fixed value $\beta_{1}=0.49$ proposed by him [63], rows $\{16 \& 23\}$ in Table S4. This leaves only one free parameter, $K_{\mathrm{a} 0}$, to be determined from the whole set of data for a given homologous series. While the result for the deviation for acids is satisfactory, for alcohols it is not.

We carried out also 3-parametric fits with SIAL, $\beta$ being given by Eq. (24), $\beta(n)=\beta_{0}+\beta_{1} n$ (rows $\{7,17 \& 24\}$ in Table S4). The results for $\beta_{1}$ of $\mathrm{C}_{n} \mathrm{H}_{2 n+1} \mathrm{OH}, \mathrm{C}_{n-1} \mathrm{H}_{2 n-1} \mathrm{COOH}$ and $\mathrm{C}_{n} \mathrm{H}_{2 n+1} \mathrm{Me}_{2} \mathrm{PO}$ are 0.56 , 
0.46 and 0.38 respectively (while according to Smith's model, $\beta_{1}$ must be the same for all series). The deviations are low - the comparison with the experimental data for alcohols is illustrated in Fig. 6 b. However, there is no correlation between cohesive behaviour and SIAL's $\beta$-values, Table 3 - the experimentally observed transition from non-cohesive to cohesive behaviour occurs at different values of $\beta(3.75,3.64$ and 4.46 for acids, alcohols and phosphineoxides respectively).

Table 3. Values of $\beta$ used for the fits with SD and SIAL models (calculated with Eq. (21) and Eq. (24), respectively).

\begin{tabular}{|c|c|c|c|c|c|c|}
\hline & \multicolumn{2}{|c|}{$\begin{array}{c}\mathrm{C}_{n-1} \mathrm{H}_{2 n-1} \mathrm{COOH} \\
21^{\circ} \mathrm{C},{ }^{\mathrm{i}} \alpha=18 \AA^{2}\end{array}$} & \multicolumn{2}{c|}{\begin{tabular}{c}
\multicolumn{2}{c|}{$\mathrm{C}_{n} \mathrm{H}_{2 n+1} \mathrm{OH}$} \\
${ }^{\circ} \mathrm{C}^{\mathrm{i}} \alpha=16.5 \AA^{2}$
\end{tabular}} & \multicolumn{2}{c|}{$\begin{array}{c}\mathrm{C}_{n} \mathrm{H}_{2 n+1} \mathrm{Me}_{2} \mathrm{PO} \\
23.5^{\circ} \mathrm{C}^{\mathrm{ii}} \alpha=28.2 \AA^{2}\end{array}$} \\
\hline$n$ & $\begin{array}{c}\beta, \\
\mathrm{SD}^{\mathrm{iii}}\end{array}$ & $\begin{array}{c}\beta, \\
\mathrm{SIAL}^{\mathrm{iv}}\end{array}$ & $\begin{array}{c}\beta, \\
\mathrm{SD}^{\mathrm{iii}}\end{array}$ & $\begin{array}{c}\beta, \\
\mathrm{SIAL}^{\mathrm{iv}}\end{array}$ & $\begin{array}{c}\beta, \\
\mathrm{SD}^{\mathrm{iii}}\end{array}$ & $\begin{array}{c}\beta, \\
\mathrm{SIAL}^{\mathrm{iv}}\end{array}$ \\
\hline 3 & ${ }^{\mathrm{nc}} 0.75$ & 2.82 & ${ }^{\mathrm{nc}} 1.01$ & 2.53 & & \\
\hline 4 & ${ }^{\mathrm{nc}} 1.32$ & 3.29 & ${ }^{\mathrm{nc}} 1.80$ & 3.08 & & \\
\hline 5 & $2.06^{\mathrm{c}}$ & 3.75 & $2.89^{\mathrm{c}}$ & 3.64 & & \\
\hline 6 & $3.00^{\mathrm{c}}$ & 4.21 & $4.35^{\mathrm{c}}$ & 4.20 & & \\
\hline 7 & $4.18^{\mathrm{c}}$ & 4.67 & $6.32^{\mathrm{c}}$ & 4.75 & ${ }^{\mathrm{nc}} 0.83$ & 2.56 \\
\hline 8 & $5.68^{\mathrm{c}}$ & 5.13 & $9.01^{\mathrm{c}}$ & 5.31 & ${ }^{\mathrm{nc}} 1.03$ & 2.94 \\
\hline 9 & $7.59^{\mathrm{c}}$ & 5.59 & $12.7^{\mathrm{c}}$ & $5.877^{\mathrm{pt}}$ & ${ }^{\mathrm{nc}} 1.26$ & 3.32 \\
\hline 10 & $10.06^{\mathrm{c}}$ & $6.05^{\mathrm{pt}}$ & $18.0^{\mathrm{c}}$ & $6.43^{\mathrm{pt}}$ & ${ }^{\mathrm{nc}} 1.49$ & 3.69 \\
\hline 11 & $13.3^{\mathrm{e}}$ & & $25.5^{\mathrm{e}}$ & & ${ }^{\mathrm{nc}} 1.75$ & 4.07 \\
\hline 12 & $17.5^{\mathrm{e}}$ & & $36.2^{\mathrm{e}}$ & & $2.02^{\mathrm{c}}$ & 4.45 \\
\hline 13 & $23.1^{\mathrm{e}}$ & & $52.0^{\mathrm{e}, \mathrm{pt}}$ & & $2.31^{\mathrm{c}}$ & 4.82 \\
\hline 14 & $30.6^{\mathrm{e}}$ & & & & $2.62^{\mathrm{c}}$ & 5.20 \\
\hline 15 & $40.8^{\mathrm{e}, \mathrm{pt}}$ & & & & $2.95^{\mathrm{c}}$ & 5.58 \\
\hline 16 & & & & & $3.30^{\mathrm{c}}$ & $5.96^{\mathrm{pt}}$ \\
\hline
\end{tabular}

${ }^{n c}$ Surfactants with non-cohesive behaviour at W|A, cf. Sec. 1.1. ${ }^{\mathrm{c}}$ Surfactant with cohesive behaviour at W|A. ${ }^{\mathrm{pt}}$

Surfactants below their critical point displaying a phase transition according to the analysis by the law of corresponding states (SD columns) or calculated from SIAL model (SIAL columns), see Sec. 3.6. ${ }^{\text {e }}$ Values are extrapolated (not based on experimental data for the respective homologue). ${ }^{i}$ Area following from crystallographic data and collapse of insoluble monolayers. ${ }^{\text {ii }}$ Area obtained as a fitting parameter from the data for the non-cohesive $\mathrm{C}_{13} \mathrm{H}_{27} \mathrm{Me}_{2} \mathrm{PO}$ in Table 2. ${ }^{\text {iii }} \mathrm{Eq}$. (21). ${ }^{\text {iv }} \mathrm{Eq}$. (24) with $\beta_{0}$ and $\beta_{1}$ from rows $\{7,17 \& 24\}$ in Table S4.

(iii) Van der Waals and Frumkin models were tested together with Eq. (24), $\beta_{\mathrm{V}}(n)=\beta_{0}+\beta_{1} n$ (due to their incompatibility with Eq. (21)). Hence, we utilized the van der Waals model with four free parameters: $K_{\mathrm{a} 0}, \alpha, \beta_{0}$ and $\beta_{1}$. The obtained area parameter is $\alpha_{\mathrm{V}}=22.5$ and $20.9 \AA^{2}$ for acids and alcohols at W|A. The values obtained for $\beta_{1}\left(0.35\right.$ and 0.39 for $\mathrm{C}_{n-1} \mathrm{H}_{2 n-1} \mathrm{COOH}$ and $\mathrm{C}_{n} \mathrm{H}_{2 n+1} \mathrm{OH}$, cf. rows $\{18 \& 25\}$ in Table S4) do not agree well with the value $\beta_{1}=0.49$ of Smith. The results obtained from our fits can be compared to those of Danov et al. [68,5,47], who also used van der Waals model but used a different 
set of experimental data and a more complex dependence $K_{\mathrm{a}}(n)$. They obtained $\beta_{1}=0.238$ and 0.304 for acids and alcohols respectively, which is lower than our values 0.35 and 0.39 . Their results for the areas $\left(\alpha_{\mathrm{V}}=22.6\right.$ and $\left.20.9 \AA^{2}\right)$ are practically equivalent to what we obtained, but this is $25 \%$ higher than the respective values following from the crystallographically determined or collapse areas.

The Frumkin model exhibits problems similar to those of van der Waals model. We obtained areas $\alpha_{\mathrm{L}}$ that are extremely high (28.8 and $31.0 \AA^{2}$ for $\mathrm{C}_{n-1} \mathrm{H}_{2 n-1} \mathrm{COOH}$ and $\mathrm{C}_{n} \mathrm{H}_{2 n+1} \mathrm{OH}$ ), and the values of $\beta_{1}$ are too low ( 0.26 for both surfactants, rows $\{19 \& 26\}$ in Table S4). These values are quite similar to those obtained by Fainerman et al. [1] - for acids they found $\ln K_{\mathrm{a}}=-20.5+1.06 n, \alpha=30 \AA^{2}, \beta_{\mathrm{L}}=-0.45$ $+0.23 n$ (following from the data for $n<12$ in their table 3.4). For alcohols, from table 3.1 in Ref. [1] we get $\ln K_{\mathrm{a}}=-20.5+1.10 n, \alpha=28 \AA^{2}, \beta_{\mathrm{L}}=-0.36+0.20 n$. These numbers suggest that Frumkin model is even less appropriate than van der Waals for analysing surface tension data.

In addition, neither vdW nor Frumkin models yield values of $\beta$ correlated to cohesive and noncohesive behaviour (the experimentally observed transition for acids, alcohols and phosphineoxides occurs at $\beta_{\mathrm{V}}=1.3,1.5,0.8$ and $\beta_{\mathrm{L}}=0.3,2.3,0.1$ respectively). Finally, unlike SD model, neither vdW nor Frumkin models yield similar values of $\alpha$ at $\mathrm{W} \mid \mathrm{A}$ and $\mathrm{W} \mid \mathrm{O}$ interface: for example, fits with free area parameter yield $\alpha=17.2, \alpha_{\mathrm{V}}=24.2, \alpha_{\mathrm{L}}=35.1 \AA^{2}$ for alcohols at $\mathrm{W} \mid \mathrm{O}$, rows $\{8,10,11\}$ in Table $\mathrm{S} 3$, while, for alcohol at W|A, $\alpha=17.3, \alpha_{\mathrm{V}}=20.9, \alpha_{\mathrm{L}}=31.0 \AA^{2}$, rows $\{22,25,26\}$ in Table S4; i.e., only SD's hard-disc area $\alpha$ does not depend on the interface.

To summarize, the application of the continual models to cohesive surfactants yields accurate results for surfactants of relatively short chain length. The SD model agrees well with the experiment with attraction parameter $\beta$ calculated from the non-linear equation (21). It is found that all surfactants which are, according to SD model and Eq. (21), above their Boyle temperatures $(\beta<2)$ behave non-cohesively and vice versa.

For completeness, we also investigated the data for the zwitterionic $\mathrm{C}_{n} \mathrm{H}_{2 n+1} \mathrm{Me}_{2} \mathrm{~N}^{+} \mathrm{CH}_{2} \mathrm{COO}^{-}$from Refs. $[93,94,83](n=8-16)$ which we discussed in Sec. 3.1. The results point at weakly cohesive behaviour with values of $\beta$ dominated by the lateral attraction between the head groups. This interesting situation is analyzed in S9.

\subsection{Phase behaviour of the cohesive monolayers}

A feature of the continual EOS (7)-(8) of Frumkin, van der Waals and SIAL is that they predict the 
existence of two phases - gaseous and liquid, provided that $\beta$ is larger than certain critical value, $\beta_{\text {cr. To }}$ analyse the phase behaviour predicted by these models, we follow Maxwell's construction for the 3-D van der Waals EOS (cf. Refs. [40,95] for a detailed exposition). The critical point of EOS (8) is determined from the condition that the critical state corresponds to a horizontal inflection of $\pi^{\mathrm{S}}(\Gamma)$, i.e. the equations $\partial \pi^{\mathrm{s}}(\Gamma ; \beta) / \partial \Gamma=0$ and $\partial^{2} \pi^{\mathrm{S}}(\Gamma ; \beta) / \partial \Gamma^{2}=0$ are fulfilled there. These two conditions are solved for $\beta$ and $\Gamma$ to yield the characteristics of the critical state according to SIAL model: $\beta_{\mathrm{cr}}=5.84$ and $\Gamma_{\mathrm{cr}}=$ $0.215 / \alpha$. Substituting these values into Eqs. (8)\&(9), we obtain the critical surface pressure and bulk surfactant concentration: $\pi_{\mathrm{cr}}^{\mathrm{S}}=0.0789 k_{\mathrm{B}} T / \alpha ; C_{\mathrm{cr}}=0.0545 / \alpha K_{\mathrm{a}}$. These values are compared in Table 4 with the respective results obtained from van der Waals model [5] and Frumkin EOS (7).

The critical value of $\beta$ can be reached by changing either the temperature or the structure of the surfactant, as it follows from Eq. (21). At low enough temperatures or for long-chained homologues where $\beta>\beta_{\mathrm{cr}}$, the EOS (8) predicts the existence of 3 regions in the $\pi^{\mathrm{S}}(\Gamma)$ dependence, divided by two extrema (as the SIAL curve with $\beta=6.66$ in Fig. 7a). The region between the extrema corresponds to unstable states of the fluid (the mechanical condition for stability, $\partial \pi^{\mathrm{S}} / \partial \Gamma>0$, is violated). On the left hand side of the minimum, a stable state of high density exist - within the continual approach, this is the LE state of the adsorption layer. On the right hand side of the maximum, a state of low density exists this is the gaseous state. The horizontal line of curve with $\beta=6.66$ in Fig. 7a connecting the two stable branches depicts the equilibrium transition from gaseous to LE state. It connects the coexistence points, located at the coexistence (binodal, or Clausius-Clapeyron) curve [40]. SIAL's binodal in Fig. 7a was calculated with the conditions for mechanical and chemical equilibrium, $\pi^{\mathrm{S}}\left(\Gamma^{\mathrm{G}}, \beta\right)=\pi^{\mathrm{S}}\left(\Gamma^{\mathrm{LE}}, \beta\right)$ and $\gamma^{\mathrm{S}}\left(\Gamma^{\mathrm{G}}, \beta\right) \Gamma^{\mathrm{G}}=\gamma^{\mathrm{S}}\left(\Gamma^{\mathrm{LE}}, \beta\right) \Gamma^{\mathrm{LE}}$. At high temperatures or short chain-lengths where $\beta<\beta_{\mathrm{cr}}$, the 2-D fluid becomes supercritical, and gaseous-liquid phase transition is absent (like the SIAL curve with $\beta=3$ in Fig. 7a).

Let us compare now these results with experimental data. The meticulous study of Kim and Cannell [25] of the phase transition gaseous-to-LE state of the insoluble $\mathrm{C}_{14} \mathrm{H}_{29} \mathrm{COOH}$ at $\mathrm{pH}=2$ can be analysed based on the principle of corresponding states and our formula (21) for $\beta$. Kim and Cannell were able to find with good precision the critical point $\left(T_{\mathrm{cr}}, \Gamma_{\mathrm{cr}}, \pi_{\mathrm{cr}}^{\mathrm{S}}\right)$ of the pentadecanoic acid. By using the value $\alpha$ $=18.0 \AA^{2}$ for the actual area per acid molecule, we obtained from their results the dimensionless critical parameters $\alpha \Gamma_{\mathrm{cr}}=0.075$ and $\alpha \pi_{\mathrm{cr}}^{\mathrm{S}} / k_{\mathrm{B}} T_{\mathrm{cr}}=0.0076$. In addition, from their critical temperature $T_{\mathrm{cr}}=$ $26.15^{\circ} \mathrm{C}$ and our Eq. (21) for $\beta$ (with $n=15$ and $\alpha=18.0 \AA^{2}$ ) we were able to estimate the respective critical value of the attraction parameter, $\beta_{\mathrm{cr}}=38.1$. This result is extremely sensitive to the value of the 
actual area: its uncertainty range ( $\alpha=18.0 \pm 0.8 \AA^{2}$ ) corresponds to $\beta_{\text {cr }}$ between 26 and 61 .

Table 4. Critical parameters of the continual adsorption models from Sec. 2.1.2 - comparison with the experimental findings of Kim and Cannell for $\mathrm{C}_{14} \mathrm{H}_{29} \mathrm{COOH}$ [25].

\begin{tabular}{|c|c|c|c|c|}
\hline & $\beta_{\text {cr }}$ & $\Gamma_{\text {cr }}$ & $\pi_{\text {cr }}^{\mathrm{S}}$ & $C_{\text {cr }}$ \\
\hline SIAL & $\beta_{\text {cr }}=5.84$ & $0.215 / \alpha$ & $0.0789 \times k_{\mathrm{B}} T / \alpha$ & $0.0545 / \alpha K_{\mathrm{a}}$ \\
\hline vdW & $\beta_{\mathrm{V}, \mathrm{cr}}=3.375$ & $0.333 / \alpha_{\mathrm{V}}$ & $0.125 \times k_{\mathrm{B}} T / \alpha_{\mathrm{V}}$ & $0.0869 / \alpha_{\mathrm{v}} K_{\mathrm{a}}$ \\
\hline Frumkin & $\beta_{\mathrm{L}, \mathrm{cr}}=2$ & $0.5 / \alpha_{\mathrm{L}}$ & $0.193 \times k_{\mathrm{B}} T / \alpha_{\mathrm{L}}$ & $0.135 / \alpha_{\mathrm{L}} K_{\mathrm{a}}$ \\
\hline experiment & ${ }^{\mathrm{a}} \beta_{\mathrm{cr}}=38.1$ & $0.075 / \alpha$ & $0.0076 \times k_{\mathrm{B}} T / \alpha$ & - \\
\hline
\end{tabular}

${ }^{a}$ The critical attraction parameter is calculated from the experimental critical temperature $T_{\mathrm{cr}}=26.15$ [25] via Eq. (21).
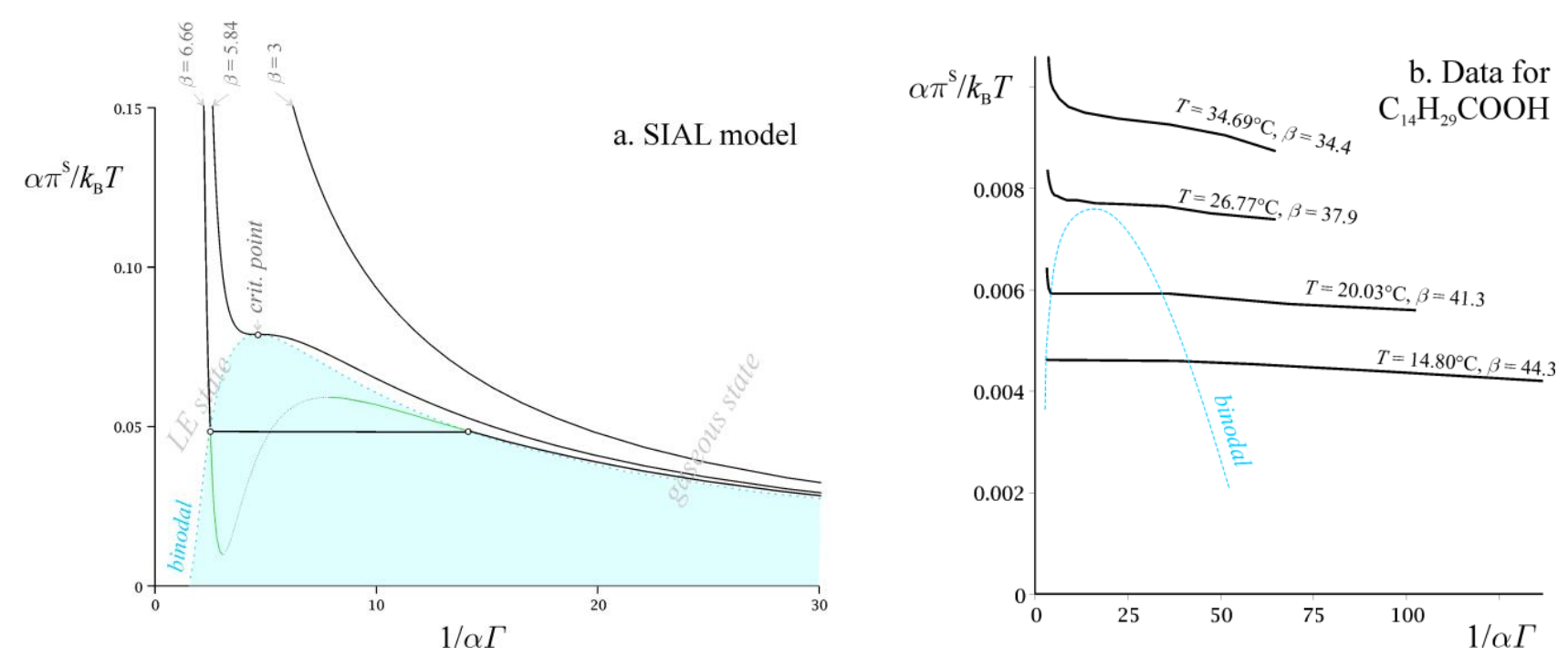

Fig. 7. Phase transition from gaseous to LE state of the monolayer. a. $\pi^{\mathrm{S}}(1 / \Gamma)$ diagram of the continual SIAL EOS (8) at different values of the attraction parameter $\beta$ (axes are non-dimensionalized for convenience). The lowest value $\beta=3$ corresponds to weakly cohesive isotherm, while $\beta=6.66$ correspond to a strongly cohesive

EOS with phase transition. The critical EOS is with $\beta=5.84$, and passes through the critical point. $\mathbf{b}$. Experimental data by Kim and Cannell for insoluble $\mathrm{C}_{14} \mathrm{H}_{29} \mathrm{COOH}$ at different temperatures. The value $\alpha=18$ $\AA^{2}$ is used to make the axes dimensionless. From each temperature, the respective $\beta$ is calculated from Eq. (21). The comparison between the two figures suggests that while SIAL model gives a qualitatively correct picture, it predicts critical point very far away from the experimental one (SIAL's $\alpha \Gamma_{\mathrm{cr}}, \alpha \pi_{\mathrm{cr}}^{\mathrm{S}} / k_{\mathrm{B}} T_{\mathrm{cr}}$ and $\beta_{\mathrm{cr}}$ differ by $\sim 1000 \%$ from the experimental values).

According to the law of corresponding states [56], the value $\beta_{\mathrm{cr}}=38.1$ must be the same for any monolayer of non-ionic surfactants, provided that there are no strong specific interactions between the head groups. This fact allows us to calculate the critical temperature of any surfactant of simple polar head group, as long as its actual area $\alpha$ is known. Thus, by solving with respect to $T$ the nonlinear 
algebraic equation $\beta(T ; n, \alpha)=\beta_{\mathrm{cr}}$, where the function $\beta(T)$ is given by Eq. (21) and $\beta_{\mathrm{cr}}=38.1$, one obtains $T_{\text {cr }}$ as a function of the chain length $n$. Using the values $\alpha=18.0,16.5$ and $28.2 \AA^{2}$ for the acids, the alcohols and the phosphineoxides respectively, one finds the following critical temperatures:

(i) Acids: $T_{\mathrm{cr}}($ tetradecanoic acid $)=3.8^{\circ} \mathrm{C} ; T_{\mathrm{cr}}($ pentadecanoic acid $)=26.3^{\circ} \mathrm{C} ; T_{\mathrm{cr}}$ (hexadecanoic acid $)$ $=49^{\circ} \mathrm{C}$.

(ii) Alcohols: $T_{\mathrm{cr}}($ dodecanol $)=17^{\circ} \mathrm{C}, T_{\mathrm{cr}}($ tridecanol $)=45^{\circ} \mathrm{C}, T_{\mathrm{cr}}($ pentadecanol $)=101^{\circ} \mathrm{C}$.

(iii) Phosphineoxides are supercritical for any realistic value of $T$ for all $n<20$, e.g., $T_{\text {cr }}$ (hexadecyldimethylphosphineoxide $)=-170^{\circ} \mathrm{C}$.

According to these results, all soluble surfactants investigated in this work form supercritical monolayers at $W \mid A$.

The above experimental results are compared in Table 4 to the theoretical critical parameters following from the models of SIAL, Frumkin and van der Waals. It is seen that all three adsorption models overestimate $\alpha \Gamma_{\text {cr }}$ and $\alpha \pi_{\mathrm{cr}}^{\mathrm{S}} / k_{\mathrm{B}} T_{\text {cr }}$ with respect to those recalculated from the data of Kim and Cannell [25]. The theoretical critical values closest to the experimental ones are expectedly those of the SIAL model. However, even they are much higher than the experimental ones - for example, the theoretical $\Gamma_{\mathrm{cr}}$ of SIAL is about 3 times higher, and $\pi_{\mathrm{cr}}^{\mathrm{S}}$ is 10 times higher than the respective experimental values. As shown in Table 3 (superscript pt), the SIAL model predicts the existence of a phase transition with nonanol, decanol, decanoic acid and hexadecyldimethylphosphineoxide (for that reason, the theoretical $\pi^{\mathrm{S}}(C)$ dependence predicted by SIAL for nonanol and decanol in Fig. 6b exhibit a small spinodal knot near the gaseous-LE transition region, cf. also S10). According to vdW model (which leads to $\left.\beta_{\mathrm{V}, \mathrm{cr}}=3.375\right)$ and the results for the $\beta_{\mathrm{V}}(n)$ dependence from rows $\{18 \& 25\}$ in Table $\mathrm{S} 4$, the first homologue displaying phase transition should be $\mathrm{C}_{10} \mathrm{H}_{21} \mathrm{OH}$ from the alkanols and $\mathrm{C}_{10} \mathrm{H}_{21} \mathrm{COOH}$ from the acids (Danov and Kralchevsky $[68,5,47]$ obtained similar results). Frumkin model $\left(\beta_{\mathrm{L}, \mathrm{cr}}=2\right)$ predicts that $\mathrm{C}_{5} \mathrm{H}_{11} \mathrm{OH}$ and $\mathrm{C}_{12} \mathrm{H}_{25} \mathrm{COOH}$ should be below their critical points. It is unlikely that such phase transition exists in reality - our results for $T_{\text {cr }}$ suggest that it is improbable that any homologue shorter than undecanol or tridecanoic acid is subcritical at any attainable temperature. All phosphineoxides, no matter what their chain lengths are, are supercritical.

The analysis from this section leads to the conclusion that the transitions from gaseous to LE state for all surfactants investigated in Sec. 3.2, $3.4 \& 3.5$ are supercritical. Therefore, the predictions of the continual models of SIAL, Frumkin and van der Waals for the phase behaviour of these surfactants are probably erroneous. Unlike SIAL model, the new EOS (10) of the SD model has no extrema, spinodal region or critical point. Rigorously, it can be used only for supercritical fluid. Indeed, the results for $T_{\text {cr }}$ 
above suggest that this is the case with the surfactants investigated in this work.

\section{Summary and conclusions}

We formulated in Sec. 1.1 several criteria to distinguish between non-cohesive and cohesive behaviour of adsorbed monolayers. In the former case the experimental dependence of the surface pressure $\pi^{\mathrm{S}}$ on concentration $C$ is a function with monotonous $1^{\text {st }}$ derivative - this is observed at water|oil and with short chained surfactants at water|air. The cohesive behaviour is characterized by a kink of the $\pi^{\mathrm{S}}(C)$ isotherm in the transition region between gaseous and liquid expanded state of the monolayer and by a negative intercept $\pi_{\text {coh }}$ of the LE region (Fig. 1). In Sec. 3.5 we found another criterion for cohesiveness: the surfactant form cohesive monolayer at W|A when it is below its Boyle temperature.

Four continual models involving lateral attraction have been compared in detail - the common models of Frumkin and van der Waals and the newer SIAL and sticky disc models. Our analysis in Sec. 2.3 of the models of Frumkin and van der Waals, Eqs. (7), revealed important shortcomings of these theories. It was shown that the respective areas per molecule $\left(\alpha_{\mathrm{L}}\right.$ and $\left.\alpha_{\mathrm{V}}\right)$ and the attraction parameters $\left(\beta_{\mathrm{L}}\right.$ and $\left.\beta_{\mathrm{V}}\right)$ these models lead to, are not uniquely defined and are usually very different from the real $\alpha$ and $\beta$. In some cases Frumkin and vdW models lead to confusing results such as negative value of the attractive parameter $[1,2,4]$. The situation is improved with the SIAL model (8)-(9) - since it is based on the almost exact EOS of HFL [48], it yields correct values of the area per molecule. In these three models the attraction between the adsorbed molecules is accounted for by the addition of an attractive term $-\beta \alpha \Gamma^{2}$ into the EOS. Such terms are valid only for binary interactions (i.e. at low surface density) and lead to poor values of the attraction parameter $\beta$. A more consistent approach to the attractive part of the EOS is the one of Ivanov et al. [15,14,7] who derived a new EOS and adsorption isotherm, Eqs. (10)-(12), in which a term for the intermolecular attraction is combined with a repulsive factor $\Gamma /(1-$

$\alpha \Gamma)^{2}$ identical to HFL's surface pressure. Since the attractive term is based on the sticky potential theory of Baxter [96] we call this model sticky disk (SD) model.

The most important criterion for the validity of the adsorption models is the consistency of the values of the adsorption parameters involved - for the continual model, these are the interaction parameters $\alpha$ and $\beta$ and the adsorption constant $K_{\mathrm{a}}$. We reviewed the models relating these parameters to the molecular characteristics of the surfactant.

The hard-disc area $\alpha$ can be determined independently from the collapse area of insoluble 
monolayers or from crystallographic data (with a correction for the packing constant). We have shown that SD model performs well at both $\mathrm{W} \mid \mathrm{O}$ and $\mathrm{W} \mid \mathrm{A}$ interface with precisely the same value of $\alpha$ (Table 2), which is not the case with the vdW and Frumkin models.

To account for the effect of the length $n$ of the interacting molecules on the attraction parameter $\boldsymbol{\beta}$ at W|A, Smith [63] and Kralchevsky et al. [68,5] assumed that $\beta$ is a linear function of $n$. A more realistic molecular model of $\beta$ was proposed in Ref. [15] and was developed further in Sec. 2.3 here. The adsorbed molecules were modelled as lines of uniform linear molecular density $1 / l_{\mathrm{CH}_{2}}$ interacting with each other with attractive London potential, leading to Eq. (21) for $\beta$. As demonstrated in Fig. 2, the resulting function $\beta(n)$ is far from being linear. We demonstrated another success of the SD model unlike any other model tested here, it agrees quantitatively with the data for alcohols, acids and phosphineoxids at W|A if Eq. (21) is used to calculate $\beta$.

The analysis of the adsorption constant $K_{\mathrm{a}}$ in Sec. 2.2 is based on the model of Ivanov et al. $[46,7,8]$. Although the final expression (13) for $K_{\mathrm{a}}$ is formally identical with that of Davies and Rideal [24], the two models are rather different: Davies and Rideal assumed that the adsorption thickness $\delta_{\mathrm{a}}$ is the length of the hydrophobic chain (which is much larger than our value $\delta_{\mathrm{a}}=0.45 \AA$ from Eq. (14)), and missed the contribution $-\alpha_{\perp} \sigma_{0}$ of the lost interface to the adsorption energy $E_{\mathrm{a}}$ (it is of the order of $-2-3 \times k_{\mathrm{B}} T$, which is by no means negligible). The two "errors" compensate each other to a certain extent, which explains the apparent success of some authors [24,5] using Davies and Rideal model. The model of $K_{\mathrm{a}}$ from Sec. 2.2 was confirmed by the results in Sec. 3.1. These are: the linearity of the dependence $\ln K_{\mathrm{a}}$ vs. $n$; the correct slopes $\Delta \mu_{\mathrm{CH}_{2}} / k_{\mathrm{B}} T=1.39$ of $\ln K_{\mathrm{a}}$ vs. $n$ for adsorption at $\mathrm{W} \mid \mathrm{O}$ and $\Delta \mu_{\mathrm{CH}_{2}} / k_{\mathrm{B}} T=1.04$ for adsorption at W|A (that follow from independent sources); the coincidence of the experimental values of the intercept $\ln K_{\mathrm{a} 0}$ with the theoretical ones, Eq. (26) (if corrected for the immersion in water of the terminal $-\mathrm{CH}_{2}-$ group polarized by the adjacent polar head group).

We further analysed the shortcomings of each of the tested adsorption models, by considering tensiometric data for several homologous series of surfactants at $\mathrm{W} \mid \mathrm{O}$. The data for $\mathrm{W} \mid \mathrm{O}$ are easier for interpretation since we have shown that $\beta$ is small and independent of $n$ (Fig. 4). We compared the four models with data for alcohols at $\mathrm{W} \mid \mathrm{O}$ using three fitting parameters $\left(K_{\mathrm{a} 0}, \alpha\right.$ and $\beta$ ). The results (rows $\{8$ 11 ) in Table S3) lead to the following main conclusions: (i) The deviations of all 3-parametric models are exactly the same, and all models yield almost the same value for the adsorption constant $K_{\mathrm{a} 0}$, very close to the one directly determined in Sec. 3.1. (ii) The SD model yields values of $\alpha$ and $\beta$ closest to the benchmark values, while the models of van der Waals and Frumkin give considerably larger values 
of $\alpha$, and negative values of $\beta$ (as can be expected from Eqs. (19)). A general conclusion from the poor results but with small deviation obtained with van der Waals and Frumkin models is that the small deviation is by no means a firm criterion for good fit.

The data for both non-cohesive and cohesive surfactants at W|A can be interpreted in the continual approach (Sec. 3). However, we found that while the continual models are well suited for non-cohesive data they fail with strongly cohesive surfactants. The SD and SIAL models applied to data for $\mathrm{C}_{n} \mathrm{H}_{2 n+1} \mathrm{Me}_{2} \mathrm{PO}, \mathrm{C}_{n} \mathrm{H}_{2 n+1} \mathrm{OH}$ and $\mathrm{C}_{n-1} \mathrm{H}_{2 n-1} \mathrm{COOH}$ exhibit good accuracy and lead to areas $\alpha$ which differ from the collapse or crystallographic areas by less than 6\%. However, while the SD model agrees also with the theoretical formula (21) for $\beta(n)$, SIAL model does not. Eq. (21) is shown to be inappropriate for surfactants with strong head group-specific lateral interaction (such as the glycines in S9). We found a correlation between the cohesive/non-cohesive behaviour and the Boyle point of the SD isotherms, which corresponds to $\beta=2$ (cf. Table 3) - the long chain homologues, which are below their Boyle temperature, are cohesive. No such correlation was found with the SIAL isotherms. The fits of W|A data with the van der Waals and Frumkin models yield again inaccurate results for the interaction parameters $\alpha$ and $\beta$.

Finally, we investigated the phase behaviour of the investigated surfactants at W|A (Sec. 3.6). The continual EOS of SIAL, vdW and Frumkin predict the existence of gaseous-LE phase transition and a critical point for several long-chained soluble surfactants (in contrast, the SD model exhibits no phase transition). The critical parameters predicted by these models are, however, very different from the ones determined from the experimental data by Kim and Cannell [25] for the insoluble surfactant $\mathrm{C}_{14} \mathrm{H}_{29} \mathrm{COOH}$ at $\mathrm{pH}=2$. Based on their experimental data and on the principle of corresponding states, as well as on our formula for $\beta$, Eq. (21), we were able to calculate the critical value of the attraction parameter, $\beta_{\mathrm{cr}}=38.1$. By solving with respect to $T$ the nonlinear algebraic equation $\beta(T ; n, \alpha)=38.1$, one obtains $T_{\mathrm{cr}}$ as a function of the chain length $n$ and the area $\alpha$. Thus we found the critical temperatures of several acids, alcohols and phosphineoxides. All soluble surfactants, investigated in this work, were shown in this way to form supercritical monolayers at W|A (above their critical temperatures). Therefore, as far as these surfactants are concerned, the lack of a phase transition in the continual SD model is not a defect.

To conclude: the continual SD model is suitable for soluble non-cohesive monolayers and the shortchained cohesive surfactants in a given series. The most important advantage of SD model is that it is the only one to be consistent - all adsorption parameters involved can be accurately predicted as functions of the surfactant structure and medium conditions. The results of it are given in Table 2, which 
is a very practical and remarkably compact summary of the experimental data for more than 30 surfactants at both $\mathrm{W} \mid \mathrm{A}$ and $\mathrm{W} \mid \mathrm{O}$ by numerous researchers. Together with Eqs. (10)-(12) of the SD model and Eqs. (25) for $K_{\mathrm{a}}$ and (21) for $\beta$, it can be used to predict $\Gamma$ and $\sigma$ with accuracy approaching the experimental one. The SD model fails with strongly cohesive films, especially near the transition point. In a companion paper, we will investigate the other theoretical approach for the description of cohesive monolayers - we will show that the partial approach is highly efficient for the LE region of cohesive surfactants, both soluble and insoluble.

\section{Acknowledgements}

R.S. acknowledges support from BP through the BP International Centre for Advanced Materials (BPICAM). The authors are grateful to Dr. Iglika Dimitrova and Dr. Iliana Delcheva for performing some of the data processing. Discussions with Dr. S. Clarke are gratefully acknowledged.

\section{Notes and references}

${ }^{1}$ This is done in two steps: first, Gibbs isotherm is used to find the respective series of the adsorption isotherm $\Gamma(C)$, and second, the obtained $\Gamma(C)$ series is substituted into the $\pi^{\mathrm{s}}(\Gamma)$ virial expansion and the resulting series is truncated to the order of $C^{2}$, which leads to Eq. (6).

${ }^{2}$ The vdW EOS was originally proposed by Volmer and Mahnert [60], but it is called 2-D van der Waals model since it is formally equivalent with the famous 3-D van der Waals equation of state.

${ }^{3}$ The exact 2-D sticky disc fluid model predicts an unphysical singularity of the equation of state [18], i.e. it is in principle impossible to find an exact EOS for such fluid.

${ }^{4}$ Note that in our previous works $[46,7,8]$, we used the symbol $\Delta u$ for the transfer energy of the surfactant from water to oil. Here we prefer the more suitable denotation $\Delta \mu$, since the transfer energy is free Gibbs (not internal) energy. The transfer energy $\Delta \mu$ is, in fact, mainly of entropic origin [65,8].

${ }^{5}$ In Ref. [8], we had no access to Aveyard and Mitchell's original work and used another source [97] which cited Aveyard and Mitchell's $K_{\mathrm{p}}$ values. Due to a technical error in this source, the $K_{\mathrm{p}}$ values there are several times larger than the original data. For this reason, the data for $\mathrm{W} \mid \mathrm{O}$ in the present Fig. 1 and Fig. 3 are shifted by a constant compared to those in fig. $2 \& 5$ in Ref. [8]. The experimental value of the intercept $\ln K_{\mathrm{a} 0}$ for alcohols at $\mathrm{W} \mid \mathrm{O}$ in table 1 in Ref. [8] is also incorrect - it must be -21.3 . 
${ }^{6}$ Note that for acids, $\mathrm{C}_{n-1} \mathrm{H}_{2 n-1} \mathrm{COOH}$, the number of carbon atoms $n$ includes also the one of the carboxylic group. Thus, when acids are considered, one must add an additional term, $-\Delta \mu_{\mathrm{CH}_{2}} / k_{\mathrm{B}} T$, in the right hand side of the expression (26) for $\ln K_{\mathrm{a} 0 \text {. }}$

\section{Electronic Supplementary Information (ESI) available:}

S1. List of symbols and abbreviations. S2. The adsorption constant $K_{\mathrm{a}}$. S3. Details about the processing of tensiometric data. S4. Calculation of the transfer energy $\Delta \mu_{\mathrm{CH} 3}$ of the methyl group. S5. The attraction parameter $\beta$. S6. Comparison of various models and fitting procedures against tensiometric data. S7. Analysis of the dispersion of the SD model as a function of the interaction parameters $\alpha$ and $\beta$. S8. Adsorption of the watersoluble non-ionic surfactants at the $\mathrm{W} \mid \mathrm{O}$ interface. S9. Application of the adsorption models to data for $\mathrm{N}$-alkylN,N-dimethylglycines at W|A. S10. Phase transition in the continual approach - the $\pi^{\mathrm{s}}(C)$ isotherm.

1 V.B. Fainerman, R. Miller, E.V. Aksenenko, A.V. Makievski. In V.B. Fainerman, D. Möbius, R. Miller (editors), Surfactants - Chemistry, Interfacial Properties, Applications. Elsevier; 2001. Chapter 3.

2 P. Warszyński, K. Lunkenheimer, J. Phys. Chem. B, 1999, 103, 4404.

3 W. Barzyk, K. Lunkenheimer, P. Warszyński, B. Jachimska, A. Pomianowski, Colloids Surfaces A, 2014, 443, 515 .

4 S. Karakashev, E. Manev, A. Nguyen, Adv. Colloid Interface Sci., 2004, 112, 31.

5 K.D. Danov, P.A. Kralchevsky, K.P. Ananthapadmanabhan, A. Lips, J. Colloid Interface Sci., 2006, 300, 809.

6 A.J. Kumpulainen, C.M. Persson, J.C. Eriksson, E.C. Tyrode, C.M. Johnson, Langmuir, 2005, $21,305$.

7 R.I. Slavchov, S.I. Karakashev, I.B. Ivanov. In L. Römsted (editor), Surfactant Science and Technology: Retrospects and Prospects. Taylor and Francis, LLC; 2014. Chapter 2.

8 R.I. Slavchov, I.M. Dimitrova, I.B. Ivanov. In R.G. Rubio, Y.S. Ryazantsev, V.M. Starov, G.X. Huang, A.P. Chetverikov, P. Arena, A.A. Nepomnyashchy, A. Ferrús, E.G. Morozov (editors), Without Bounds: a Scientific Canvas of Nonlinearity and Complex Dynamics. Springer-Verlag; 2013, p. 199.

9 M.M. Hossain, T. Suzuki, K. Iimura, T. Kato, Langmuir, 2006, 22, 1074.

10 V.M. Kaganer, H. Möhwald, P. Dutta, Rev. Mod. Phys., 1999, 71, 779.

11 K.B. Eisenthal, Chem. Rev., 1996, 96, 1343.

12 D. Vollhardt, V.B. Fainerman, Adv. Colloid Interface Sci., 2010, 154, 1.

13 H. Watarai, N. Teramae, T. Sawada (editors), Interfacial Nanochemistry. Kluwer Academic/Plenum Publishers; 2005.

14 T.D. Gurkov, I.B. Ivanov. In Proc. 4th World Congress on Emulsions, Lyon, 2006, p. 509.

15 I.B. Ivanov, K.D. Danov, D. Dimitrova, M. Boyanov, K.P. Ananthapadmanabhan, A. Lips, Colloids Surfaces A, 2010, 354, 118. 
A.I. Rusanov, Colloid J., 2007, 69, 131.

17 K. Lunkenheimer, R. Hirte, J. Phys. Chem., 1992, 96, 8683.

18 A. Mulero (editor), Theory and Simulation of Hard-Sphere Fluids and Related Systems. Springer; 2008.

19 N.K. Adam, The Physics and Chemistry of Surfaces. Clarendon Press; 1941.

20 I. Langmuir, J. Chem. Phys., 1933, 1, 756.

21 D.K. Chattoraj, K.S. Birdi, Adsorption and the Gibbs Surface Excess. Plenum press; 1984. Chapters 5\&6.

22 N.K. Adam, Proc. Roy. Soc. A, 1922, 101, 516.

23 J.T. Davies, J. Colloid Sci., 1956, 11, 377.

24 J.T. Davies, E. Rideal, Interfacial Phenomena. Academic Press; 1963. Chapters 4\&5.

25 M.W. Kim, D.S. Cannell, Phys. Rev. A, 1976, 13, 411.

26 D. Hönig, D. Möbius, J. Phys. Chem., 1991, 95, 4590.

27 M. Lösche, E. Sackmann, H. Möhwald, Ber. Bunsenges Phys. Chem., 1983, 87, 848.

28 M. Thoma, T. Pfohl, H. Möhwald, Langmuir, 1995, 11, 2881.

29 R. Aveyard, B.J. Briscoe, J. Chem. Soc. Faraday Trans. 1, 1972, 68, 478.

30 R. Aveyard, R.W. Mitchell, Trans. Faraday Soc., 1969, 65, 2645.

31 R. Aveyard, B.J. Briscoe, J. Chapman, J. Chem. Soc. Faraday Trans. 1, 1973, 69, 1772.

32 R. Miller, J. Krägel, V.B. Fainerman, A.V. Makievski, D.O. Grigoriev, F. Ravera, L. Liggieri, D.Y. Kwok, A.W. Neumann. In J. Sjoblom (editor), Encyclopedic Handbook of Emulsion Technology. Marcel Dekker 2001. Chapter 1.

33 F.S. Manning, R.E. Thompson, Oilfield Processing vol. 2: Crude Oil. Penn Well; 1995. Chapters $4 \& 6$.

34 J. Sjoblom (editor), Encyclopedic Handbook of Emulsion Technology. Marcel Dekker 2001.

35 T. Austad, Water-based EOR in carbonates and sandstones: new chemical understanding of the EOR potential using smart water. Enhanced oil recovery Field case studies, 2013; pp 301-335.

36 R.M. Mortier, M.F. Fox, S.T. Orszuluk (editors), Chemistry and technology of lubricants, 3rd ed. Springer; 2010. Chepters $3 \& 6$.

37 T.P. Vishnyakova, I.A. Golubeva, I.F. Krilov, O.P. Likov, Stabilizers and modificators of petroleum distillate fuels. Khimiya; 1990. Chapter 4 (in Russian).

38 J. Barker, C. Snape, D. Scurr, SAE 2014-01-1387.

39 A. Klinkenberg, J.L. van der Minne, Electrostatics in the Petroleum industry. Cahp CI, Elsevier, Amsterdam; 1958.

40 D. Kashchiev, Nucleation - Basic Theory with Applications. Butterworth-Heinemann; 2000. Chapter 1.

41 E.A. Guggenheim, Thermodynamics. Elsevier; 1967.

42 J. Thomson, Proc. Roy. Soc., 1871, 20, 1.

43 G. Jura, W.D. Harkins, J. Chem. Phys., 1944, 12, 113.

44 J. Traube, Liebigs. Ann., 1891, 265, 27. 
45 I. Langmuir, J. Amer. Chem. Soc., 1917, 39, 1848.

46 I.B. Ivanov, K.P. Ananthapadmanabhan, A. Lips, Adv. Colloid Interface Sci., 2006, 123-126, 189.

47 K.D. Danov, P.A. Kralchevsky, Colloid J., 2012, 74, 172.

48 E. Helfand, H.L. Frisch, J.L. Lebowitz, J. Chem. Phys., 1961, 34, 1037.

49 E.T. Denisov, I.B. Afanas'ev, Oxidation and antioxidants in organic chemistry and biology. CRC Taylor \& Francis; 2005.

50 J.R. Hommelen, J. Colloid Science, 1959, 14, 385.

51 S.Y. Lin, K. McKeigue, C. Maldarelli, Langmuir, 1991, 7, 1055.

52 S.Y. Lin, T.L. Lu, W.B. Hwang, Langmuir, 1994, 10, 3442.

53 S.Y. Lin, Y.C. Lee, M.W. Yang, H.S. Liu, Langmuir, 2003, 19, 3164.

54 F.P. Buff, F.H. Stillinger. In A.N. Frumkin (editor), The Basic Problems in the Modern Theoretical Electrochemistry. Mir; 1965, in Russian.

55 I. Langmuir, J. Amer. Chem. Soc., 1918, 40, 1361.

56 T.L. Hill, An Introduction to Statistical Thermodynamics. Addison-Wesley; 1962.

57 M. Volmer, Z. Phys. Chem., 1925, 115, 253.

58 L. Tonks, Phys. Rev., 1936, 50, 955.

59 A. Frumkin, Z. Phys. Chem., 1925, 116, 466.

60 M.A. Volmer, P. Mahnert, Z. Phys. Chem., 1925, 115, 236.

61 J.H. De Boer, The Dynamical Character of Adsorption. Clarendon Press; 1953.

62 J.N. Israelachvili, Intermolecular and Surface Forces, $3^{\text {rd }}$ ed. Academic Press; 2011.

63 T. Smith, J. Colloid Interface Sci., 1967, 23, 27.

64 L.D. Landau, E.M. Lifshitz, Theoretical physics, Vol. V: Statistical physics, part 1, $3^{\text {rd }}$ ed. Nauka; 1976 \& Pergamon Press; 1980.

65 C. Tanford, The Hydrophobic Effect. Wiley; 1980.

66 J.O. Hirschfelder, C.F. Curtiss, R.B. Bird, Molecular Theory of Gases and Liquids. Wiley; 1954.

67 A.I. Rusanov, Colloids Surf. A, 2004, 239, 105.

68 P.A. Kralchevsky, K.D. Danov, V.L. Kolev, G. Broze, A. Mehreteab, Langmuir, 2003, 19, 5004.

69 A.K. Chatterjee, D.K. Chattoraj, J. Colloid Interface Sci., 1968, 26, 1.

70 W. Hückel, Theoretical Principles of Organic Chemistry, Vol. II, p. 435. Elsevier; 1958.

71 A.I. Kitaigorodskii, Organic Chemical Crystallography. Consultant Bureau; 1961.

72 H. Lange, P. Jeschke. In M.J. Schick (editor), Non-ionic surfactants. Physical chemistry. Marcel Dekker; 1987.

73 M.H. Abraham, J. Chem. Soc. Faraday Trans. 1, 1984, 80, 153.

74 C. Tanford. In K.L. Mittal (editor), Micellization, Solubilization and Microemulsions, Vol. 1-2. Plenum Press; 1977. 
75 P. Kruglyakov, Hydrophile-Lipophile Balance of Surfactants and Solid Particles. Elsevier; 2000.

76 R. Vochten, G. Petre, J. Colloid lnterface Sci., 1973, 42, 320.

77 N.A. Grozev, T.G. Tosheva, I.M. Dimitrova, R.I. Slavchov, Bulg. J. Chem., 2013, $2,25$.

78 A. Ben-Haim, Hydrophobic Interactions. Plenum Press; 1980.

79 R. Aveyard, S.M. Saleem, R. Heselden, J. Chem. Soc. Faraday Trans. I, 1977, 73, 84.

80 M. Ferrari, L. Liggieri, F. Ravera, C. Amodio, R. Miller, J. Colloid lnterface Sci., 1996, 186, 40.

81 A.V. Makievski, D. Grigoriev, Colloid Surf. A, 1998, 143, 233.

82 K. Lunkenheimer, K. Haage, R. Miller, Colloids Surf., 1987, 22, 215.

83 V.B. Fainerman, S.V. Lylyk, Koll. Zh., 1983, 45, 500.

84 D.O. Grigoriev, O. Senkel, G. Kretzschmar, J.B. Li, B.A. Noskov, R. Miller, Colloids Surf. A, 1999, $149,81$.

85 C.C. Addison, J. Chem. Soc., 1945, 98.

86 M. Aratono, S. Uryu, Y. Hayami, K. Motomura, R. Matuura, J. Colloid Interface Sci., 1984, 98, 33.

87 R. Wüstneck, R. Miller, Colloids Surf., 1990, 47, 15.

88 R.W. Grabner, C.W. Clump, J. Chem. Eng. Data, 1965, 10, 13.

89 V.D. Dolzhikova, Y.V. Goryunov, B.D. Summ, Colloid J., 1982, 44, 560.

90 K. Lunkenheimer, W. Barzyk, R. Hirte, R. Rudert, Langmuir, 2003, 19, 6140.

91 K. Malysa, R. Miller, K. Lunkenheimer, Colloids Surf., 1991, 53, 47.

92 E.H. Lucassen-Reynders, J. Colloid Interface Sci., 1972, 41,156.

93 R. Wüstneck, J. Kriwanek, M. Herbst, G. Wasow, K. Haage, Colloids Surf., 1992, 66, 1.

94 R. Wüstneck, R. Miller, J. Kriwanek, H.R. Holzbauer, Langmuir, 1994, 10, 3738.

95 R. Defay, I. Prigogine, Surface tension and adsorption. Longmans, Green and Co.; 1966. Chapter 14.

96 R.J. Baxter, J. Chem. Phys., 1968, 49, 2770.

97 F. Ravera, M. Ferrari, L. Liggieri, Adv. Colloid Interface Sci., 2000, 88, 129. 


\section{Adsorption Parameters and Phase Behaviour of Non-Ionic Surfactants at Liquid Interfaces}

(supplementary information)

\section{Radomir Iliev Slavchov ${ }^{\#, *}$, Ivan Boyanov Ivanov ${ }^{\sharp}$}

\# Department of Chemical Engineering and Biotechnology, Cambridge University, UK, CB3 OAS Cambridge,

Philippa Fawcett Drive, West Site

E-mail: ris26@cam.ac.uk

$\$$ Laboratory of Chemical Physics and Engineering, Faculty of Chemistry and Pharmacy, Sofia University,

1164 Sofia, Bulgaria

E-mail: ii@lcpe.uni-sofia.bg

\section{S1. List of symbols and abbreviations}

$B_{2} \quad$ second virial coefficient

C surfactant concentration in the bulk

$E_{\mathrm{a}} \quad$ adsorption free energy

$k_{\mathrm{B}} \quad$ Boltzmann constant

$K_{\mathrm{a}} \quad$ Henry's adsorption constant $\left(\Gamma \stackrel{\hookrightarrow \rightarrow 0}{\rightarrow} K_{\mathrm{a}} C\right)$

$L_{\mathrm{CH}_{2}} \quad$ London constant for two $-\mathrm{CH}_{2}-$ groups interacting through air

$l_{\mathrm{CH}_{2}} \quad$ length per $-\mathrm{CH}_{2}-$ group $(1.26 \AA)$

$n$ number of carbon atoms in the hydrocarbon chain of a surfactant

$T$ temperature

$z \quad$ Cartesian coordinate

$\alpha \quad$ actual area of a molecule

$\beta \quad$ attraction parameter

$\beta_{1} \quad \partial \beta / \partial n$ in Smith's linear equation for $\beta(n)$

$\Gamma \quad$ surfactant adsorption

$\gamma \quad$ activity coefficient

$\delta_{\mathrm{a}} \quad$ adsorption length 
$\Delta \mu_{\mathrm{CH}_{2}}$ free energy for transfer of $-\mathrm{CH}_{2}-$ from the hydrophobic phase to water

$\mu \quad$ chemical potential

$\mu_{0} \quad$ standard chemical potential

$\sigma \quad$ surface/interfacial tension

$\sigma 0$ interfacial tension of the pure interface in the absence of surfactant

$\pi_{\text {coh }} \quad$ cohesive pressure

$\pi^{\mathrm{S}} \quad$ surface pressure, $\sigma_{0}-\sigma$

B bulk

cr parameter of the critical point

EOS equation of state

HFL Helfand-Frisch-Lebowitz model, Eqs. (1)\&(4)

$\mathrm{L} \quad$ parameter of the Langmuir and Frumkin models

LE liquid expanded state of the adsorption layer

S surface

SIAL Smith-Ivanov-Ananthapadmanabhan-Lips model, Eqs. (8)-(9)

SD sticky disc model, Eqs. (10)-(12)

$\mathrm{V}$ parameter of the Volmer, vdW and Langmuir-Volmer LE models

vdW 2-dimensional van der Waals model, Eq. (7)

W|A water-air interface

$\mathrm{W} \mid \mathrm{O} \quad$ water-oil interface

$-\mathrm{CH}_{2}-$ methylene group

$-\mathrm{CH}_{3}$ methyl group

$\mathrm{C}_{n} \mathrm{H}_{2 n+1} \mathrm{OH} \quad$ alkan-1-ol

$\mathrm{C}_{n-1} \mathrm{H}_{2 n-1} \mathrm{COOH} \quad$ alkanoic acid

$\mathrm{C}_{n} \mathrm{H}_{2 n+1} \mathrm{Me}_{2} \mathrm{PO} \quad \mathrm{n}$-alkyl dimethyl phosphine oxides

$\mathrm{C}_{n} \mathrm{H}_{2 n+1} \mathrm{Me}_{2} \mathrm{~N}^{+} \mathrm{CH}_{2} \mathrm{COO}^{-} \quad \mathrm{N}$-n-alkyl-N,N-dimethylglycine 
Table S1. Equations of state and surface activity coefficients (adsorption isotherms) of various adsorption models.

\begin{tabular}{|c|c|c|}
\hline \multicolumn{3}{|c|}{ Continual models } \\
\hline model & $\pi^{\mathrm{S}} / k_{\mathrm{B}} T(\mathrm{EOS})$ & $\ln \gamma^{\mathrm{S}}\left(\right.$ adsorption isotherm $\left.K_{\mathrm{a}} C=\gamma^{\mathrm{S}} \Pi\right)$ \\
\hline Henry & $\Gamma$ & 0 \\
\hline virial & $\begin{array}{c}\Gamma+B_{2} \Gamma^{2}+\mathrm{O}\left(\Gamma^{3}\right), \\
K_{\mathrm{a}} C-B_{2}\left(K_{\mathrm{a}} C\right)^{2}+\mathrm{O}\left(C^{3}\right)\end{array}$ & $2 B_{2} \Gamma+\mathrm{O}\left(\Gamma^{2}\right)$ \\
\hline HFL & $\frac{\Gamma}{(1-\alpha \Gamma)^{2}}$ & $-\ln (1-\alpha \Gamma)+\frac{\alpha \Gamma(3-2 \alpha \Gamma)}{(1-\alpha \Gamma)^{2}}$ \\
\hline SIAL & $\frac{\Gamma}{(1-\alpha \Gamma)^{2}}-\beta \alpha \Gamma^{2}$ & $-\ln (1-\alpha \Gamma)+\frac{\alpha \Gamma(3-2 \alpha \Gamma)}{(1-\alpha \Gamma)^{2}}-2 \beta \alpha \Gamma$ \\
\hline SD & $\begin{array}{c}\frac{R_{\beta}-1}{2 \alpha \beta(1-\alpha \Gamma)}, \\
R_{\beta}=\sqrt{1+4 \beta \frac{\alpha \Gamma}{1-\alpha \Gamma}}\end{array}$ & $\begin{aligned}-\ln (1-\alpha \Gamma)+\left(2+\frac{1}{\beta}\right) \ln \frac{2}{1+R_{\beta}} \\
+\frac{\alpha \Gamma(4-3 \alpha \Gamma)}{(1-\alpha \Gamma)^{2}} \frac{2}{1+R_{\beta}}\end{aligned}$ \\
\hline Volmer & $\frac{\Gamma}{1-\alpha_{\mathrm{v}} \Gamma}$ & $-\ln \left(1-\alpha_{\mathrm{v}} \Gamma\right)+\frac{\alpha_{\mathrm{v}} \Gamma}{1-\alpha_{\mathrm{v}} \Gamma}$ \\
\hline vdW & $\frac{\Gamma}{1-\alpha_{\mathrm{v}} \Gamma}-\beta_{\mathrm{v}} \alpha_{\mathrm{v}} \Gamma^{2}$ & $-\ln \left(1-\alpha_{\mathrm{v}} \Gamma\right)+\frac{\alpha_{\mathrm{v}} \Gamma}{1-\alpha_{\mathrm{v}} \Gamma}-2 \beta_{\mathrm{v}} \alpha_{\mathrm{v}} \Gamma$ \\
\hline Langmuir & $-\frac{\ln \left(1-\alpha_{\mathrm{L}} \Gamma\right)}{\alpha_{\mathrm{L}}}$ & $-\ln \left(1-\alpha_{\mathrm{L}} \Gamma\right)$ \\
\hline Frumkin & $-\frac{\ln \left(1-\alpha_{\mathrm{L}} \Gamma\right)}{\alpha_{\mathrm{L}}}-\beta_{\mathrm{L}} \alpha_{\mathrm{L}} \Gamma^{2}$ & $-\ln \left(1-\alpha_{\mathrm{L}} \Gamma\right)-2 \beta_{\mathrm{L}} \alpha_{\mathrm{L}} \Gamma$ \\
\hline
\end{tabular}




\section{S2. The adsorption constant $K_{\mathrm{a}}$}

In this section, the adsorption constant $K_{\mathrm{a}}$ will be related to the surfactant structure and the parameters of the media (temperature, bulk compositions etc.) through a model proposed by Ivanov et al. [46,7,8] for the interaction free energy $\Delta \mu(z)$ of a surfactant molecule with the interface. The main contributions to $\Delta \mu(z)$ which were taken into account in this model are summarized below.

a) Contribution of the hydrophobic solvation of surfactant's linear hydrocarbon chain. The adsorption of a surfactant molecule from the water phase to the interface is due mostly to the hydrophobic effect $[65,62]$, i.e. to the change of the free energy of the hydrophobic tail upon its transfer from the hydrophobic phase to the water phase. Let this transfer energy per single $-\mathrm{CH}_{2}-$ group be $\Delta \mu_{\mathrm{CH}_{2}}\left(\Delta \mu_{\mathrm{CH}_{2}}\right.$ $>0)$. We will denote the length of a $-\mathrm{CH}_{2}-$ group along the hydrophobic chain by $l_{\mathrm{CH}_{2}}\left(l_{\mathrm{CH}_{2}}=1.26 \AA\right.$ $[62,65]$ ) and the number of the carbon atoms in the hydrocarbon chain by $n$ (therefore, the total chain length, including the $-\mathrm{CH}_{3}$ end, is close to $n l_{\mathrm{CH}_{2}}$ ). We will use the symbol $z$ for the distance between the interface and the "hydrophilic-lyophilic centre" of the surfactant, which is the point where the hydrophilic head and the hydrophobic chain are connected [75,65]. For simplicity, we assume that the surfactant molecule remains perpendicular to the interface during the adsorption process. Then, if $z<$ $n l_{\mathrm{CH}_{2}}$, a portion of the surfactant hydrocarbon chain of length $n l_{\mathrm{CH}_{2}}-z$ will be immersed into the hydrophobic phase. This corresponds to a free energy change $-\left(n-z / l_{\left.\mathrm{CH}_{2}\right)}\right) \Delta \mu_{\mathrm{CH}_{2}}$ upon transfer of a molecule from the bulk to a distance $z$ from the interface [46]. The result is, however, not entirely correct, since the end $-\mathrm{CH}_{3}$ group has different area and adsorption energy from a- $-\mathrm{CH}_{2}-$ group. We account for this by introducing in the energy $-\left(n-z / l_{\mathrm{CH}_{2}}\right) \Delta \mu_{\mathrm{CH}_{2}}$ a correction term $\Delta \mu_{\mathrm{CH}_{3}}-\Delta \mu_{\mathrm{CH}_{2}}$ equal to the extra adsorption energy of the methyl group with respect to a $-\mathrm{CH}_{2}-$. This yields [46]:

$$
\Delta \mu_{\mathrm{h}}(z)=\left\{\begin{array}{cc}
-\Delta \mu_{\mathrm{CH}_{3}}-\left(n-1-z / l_{\mathrm{CH}_{2}}\right) \Delta \mu_{\mathrm{CH}_{2}}, & z<n l_{\mathrm{CH}_{2}} \\
0, & z>n l_{\mathrm{CH}_{2}}
\end{array}\right.
$$

b) Contribution of the disappearing interfacial area. Upon adsorption, the hydrocarbon chain penetrates the interface, and a portion of the interface is replaced by the chain $[46,7,6]$. If the crosssectional area of the chain is $\alpha \perp$, then the contribution of this replacement to the adsorption energy is equal to $-\alpha_{\perp} \sigma_{0}$, where $\sigma_{0}$ is the interfacial tension of the pure interface. Assuming that this energy is gained at the moment of contact between the hydrocarbon chain and the interface (at $z=n l_{\mathrm{CH}_{2}}$ ), we can write the corresponding potential profile $\Delta \mu_{\sigma}$ as [46]: 


$$
\Delta \mu_{\sigma}(z)=\left\{\begin{array}{cc}
-\alpha_{\perp} \sigma_{0}, & z<n l_{\mathrm{CH}_{2}} \\
0, & z>n l_{\mathrm{CH}_{2}}
\end{array}\right.
$$

The significance of this term for the dependence of the adsorption constant $K_{\mathrm{a}}$ at $\mathrm{W} \mid \mathrm{O}$ interface on the nature of the oil phase is discussed in Ref. [7]. The linear dependence of $\ln K_{\mathrm{a}}$ on $\sigma_{0}$ was confirmed there by using data of Rehfeld [98] for the adsorption isotherms of the ionic surfactant sodium dodecylsulfate on various $\mathrm{W} \mid \mathrm{O}$ interfaces which allow determination of $K_{\mathrm{a}}$ as a function of $\sigma_{0}$ only. The term $\alpha_{\perp} \sigma_{0}$ has a large contribution to the enthalpy of adsorption, and therefore, determines to a large extent the temperature dependence of $K_{\mathrm{a}}$ (figure 8 in Ref. [8]).

c) Contribution of rotation. In order to estimate the contribution of the rotational degrees of freedom of the adsorbed molecule to the adsorption constant $K_{\mathrm{a}}$, let us first calculate the partition functions for the initial (in the bulk) and the final (at the surface) states. For simplicity we assume that the molecule rotates as a solid stick of inertial moment $I$. The Hamiltonian of a freely rotating stick in spherical $(r, \vartheta, \varphi)$ coordinates is [56]:

$$
H=\left(p_{\vartheta}^{2}+p_{\varphi}^{2} / \sin ^{2} \vartheta\right) / 2 I .
$$

Here $p \vartheta$ and $p_{\varphi}$ are the respective momenta of the stick. This Hamiltonian corresponds to bulk partition function (Eq. 8-27 of Hill [56]):

$$
q_{\mathrm{rot}}^{\mathrm{B}}=\frac{1}{h^{2}} \int_{-\infty}^{\infty} \int_{-\infty}^{\infty} \int_{0}^{\pi} \int_{0}^{2 \pi} \mathrm{e}^{-H / k_{\mathrm{B}} T} \mathrm{~d} \varphi \mathrm{d} \vartheta \mathrm{d} p_{\varphi} \mathrm{d} p_{\vartheta}=8 \pi^{2} k_{\mathrm{B}} T I / h^{2},
$$

where $h$ is Planck constant. For a surfactant molecule at the interface, we assume that this rotation is again free but restricted to the semi-space $z<0$. This yields for the surface partition function $q_{\mathrm{rot}}^{\mathrm{S}}$ :

$$
q_{\mathrm{rot}}^{\mathrm{S}}=\frac{1}{h^{2}} \int_{-\infty}^{\infty} \int_{-\infty}^{\infty} \int_{0}^{\pi / 2} \int_{0}^{2 \pi} \mathrm{e}^{-H / k_{\mathrm{B}} T} \mathrm{~d} \varphi \mathrm{d} \vartheta \mathrm{d} p_{\varphi} \mathrm{d} p_{\vartheta}=4 \pi^{2} k_{\mathrm{B}} T I / h^{2} .
$$

It follows from Eqs. (33)\&(34) that when the molecule is far from the interface, the free energy of rotation is:

$$
f_{\text {rot }}\left(z>n l_{\mathrm{CH}_{2}}\right)=-k_{\mathrm{B}} T \ln \left(4 \pi^{2} k_{\mathrm{B}} T I / h^{2}\right),
$$

and when it is precisely at $z=0$,

$$
f_{\text {rot }}(z=0)=-k_{\mathrm{B}} T \ln \left(2 \pi^{2} k_{\mathrm{B}} T I / h^{2}\right) .
$$

Upon transfer to $z=0$, the surfactant molecule is losing total rotational free energy $\Delta f_{\text {rot }}(z=0)=$ $f_{\text {rot }}(z=0)-f_{\text {rot }}\left(z>n l_{\mathrm{CH}_{2}}\right)=k_{\mathrm{B}} T \ln 2$. The local shape of $f_{\text {rot }}(z)$ in the interval $n l_{\mathrm{CH}_{2}}>z>0$ is relatively unimportant for the final result for $K_{\mathrm{a}}$, except for too short-chained surfactants. Therefore, for the sake 
of simplicity, similarly to Eqs. (30)\&(31), we approximate $\Delta f_{\text {rot }}$ with a simple step-function:

$$
\Delta \mu_{\mathrm{rot}}=\left\{\begin{array}{cc}
k_{\mathrm{B}} T \ln 2, & z<n l_{\mathrm{CH}_{2}} \\
0, & z>n l_{\mathrm{CH}_{2}}
\end{array}\right.
$$

The difference between $\Delta f_{\text {rot }}$ and $\Delta \mu_{\text {rot }}$ is disregarded.

d) Contribution of the hydration of the head group. We assume that the head group cannot be dehydrated and desorbed into the hydrophobic phase. This is equivalent to a hard-wall potential: $\Delta \mu(z)$ $=\infty$ at $z<0$.

e) Other contributions. There are other factors, which also contribute to the adsorption energy: (i) The interaction between the hydrophilic head group and the interface at $z>0$; (ii) Appearance of an induced dipole moment involving the $-\mathrm{CH}_{2}-$ group adjacent to the polar hydrophilic head, which acts oppositely to the hydrophobic effect and leads to immersion of the methylene group into the water phase (cf. chap. 3 of Ref. [65]). (iii) Changes in the internal degrees of freedom (vibration and internal rotation) of the molecule upon adsorption. The latter effect is involved to a certain extent in the transfer energy $n \Delta \mu_{\mathrm{CH}_{2}}$ of the surfactant chain from the hydrophobic phase to the water and since we are using experimental values for $\Delta \mu_{\mathrm{CH}_{2}}$, it is probably accounted for implicitly in our model. (iv) The approximated nature of our equations for the contributions a-d) can also affect the final result for the adsorption energy. Since the effects (i-iv) are still not well-understood, we account for them by adding to the total adsorption energy of the surfactant an empirical constant $\Delta \mu_{\text {head }}$ encompassing all of them its contribution will be analysed post-factum, by comparing theoretical results with the experimental data (Sec. 3.1).

Combining Eqs. (30),(31)\&(37) with the contributions d) and e), one obtains for $\Delta \mu(z)$ :

$$
\Delta \mu(z)=\left\{\begin{array}{cc}
\infty, & z<0 ; \\
-E_{\mathrm{a}}+k_{\mathrm{B}} T \ln 2+\Delta \mu_{\mathrm{CH}_{2}} z / l_{\mathrm{CH}_{2}}, & z<n l_{\mathrm{CH}_{2}} \\
0, & z>n l_{\mathrm{CH}_{2}} .
\end{array}\right.
$$

Here the adsorption free energy $E_{\mathrm{a}}$ stands for the expression:

$$
E_{\mathrm{a}}=(n-1) \Delta \mu_{\mathrm{CH}_{2}}+\Delta \mu_{\mathrm{CH}_{3}}+\alpha_{\perp} \sigma_{0}+\Delta \mu_{\text {head }} .
$$

Eq. (38) is illustrated in Fig. S1. 


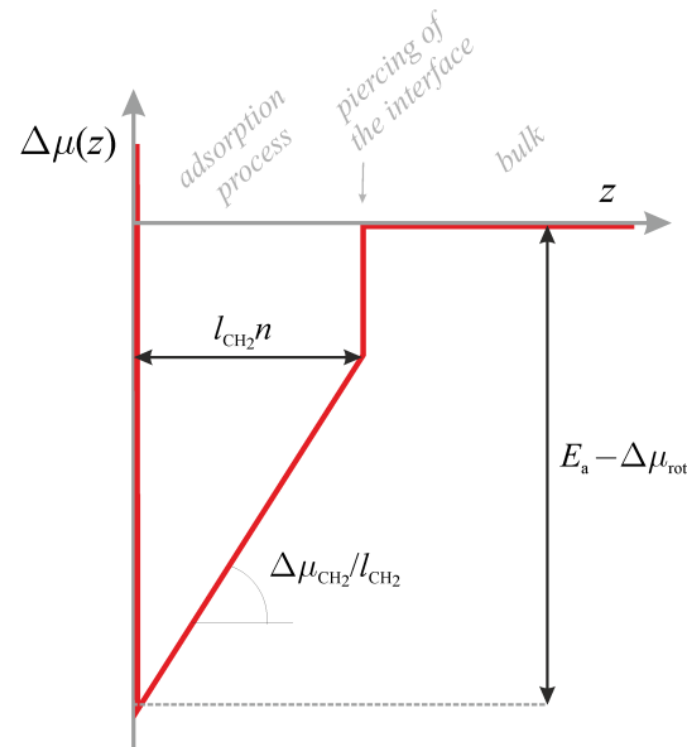

Fig. S1. Interaction potential $\Delta \mu(z)$ between a surfactant molecule and the interface as a function of the distance $z$ between the surfactant hydrophilic-lyophilic centre and the interface, cf. Eq. (38). According to the model, at distance $z>n l_{\mathrm{CH}_{2}}$, there is no significant interaction. At $z=n l_{\mathrm{CH}_{2}}$, energy is gained due to the disappearance of clean water surface of area $\alpha_{\perp}$, and the transfer energy of the $-\mathrm{CH}_{3}$ group, cf. Eq. (31). At shorter distances, there is a linear dependence of $\Delta \mu$ on $z$ related to the energy of transfer $n \Delta \mu_{\mathrm{CH}_{2}}$ of the hydrocarbon chain from water to the hydrophobic phase, see Eq. (30).

Let us now consider an ideal surfactant solution of concentration $C$. The local chemical potential $\mu(z)$ of a molecule at a distance $z$ from the interface is:

$$
\mu(z)=\mu_{0}^{\mathrm{B}}+k_{\mathrm{B}} T \ln C^{\mathrm{S}}(z)+\Delta \mu(z) .
$$

Here, $C^{\mathrm{S}}(z)$ is the local concentration of surfactant near the surface. From the condition $\mu(z)=\mu^{\mathrm{B}}=$ $\mu_{0}^{\mathrm{B}}+k_{\mathrm{B}} T \ln C$ for chemical equilibrium, the Boltzmann distribution of the surfactant molecules follows:

$$
C^{\mathrm{S}}(z)=C \exp \left(-\Delta \mu(z) / k_{\mathrm{B}} T\right) .
$$

Inserting this distribution into Gibbs definition of adsorption, one obtains Henry's adsorption isotherm:

$$
\Gamma=\int_{0}^{\infty}\left(C^{\mathrm{S}}(z)-C\right) \mathrm{d} z \equiv K_{\mathrm{a}} C,
$$

where the adsorption constant of the surfactant $K_{\mathrm{a}}$ is defined as:

$$
K_{\mathrm{a}}=\int_{0}^{\infty}\left[\exp \left(-\Delta \mu(z) / k_{\mathrm{B}} T\right)-1\right] \mathrm{d} z
$$

It follows from this derivation that Henry's isotherm (42) is valid if the surface-molecule interaction potential $\Delta \mu(z)$ is independent on $C(z)$, which is the case of dilute adsorption layer. Inserting the 
expression (38) for $\Delta \mu(z)$ into the definition (43) of $K_{\mathrm{a}}$ and performing the integration, one obtains:

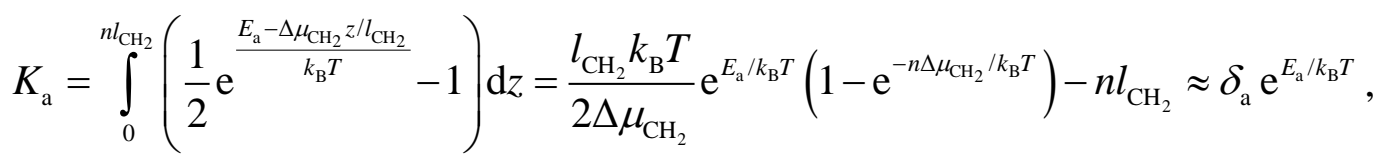

i.e. Eq (13). The pre-exponential factor $\delta_{\mathrm{a}}$ has the dimension of length and we call it the adsorption length. Our definition (14) of $\delta_{\mathrm{a}}$ is similar to the one in the theory of adsorption of spherical molecules [56] in the sense that it involves only factors related to the kinetic energy of the adsorbed molecules. Eq.

(13) is determining also the value of $\mu_{0}^{\mathrm{S}}$ in Eq. (2): $\mu_{0}^{\mathrm{S}}=\mu_{0}^{\mathrm{B}}-k_{\mathrm{B}} T \ln K_{\mathrm{a}}=\mu_{0}^{\mathrm{B}}-E_{\mathrm{a}}-k_{\mathrm{B}} T \ln \delta_{\mathrm{a}}$, cf. also Eq. (3).

\section{S3. Details about the processing of tensiometric data}

Here we describe the procedures for processing the experimental tensiometric data and determining the values of the adsorption constants of non-ionic surfactants within the direct approach (Sec. 3.1). Within the direct approach, this is done by fitting the initial region of the experimental surface pressure isotherms with the virial expansion (6) of $\pi^{\mathrm{S}}(C)$.

Let us consider first the adsorption at $\mathrm{W} \mid \mathrm{O}$. Aveyard and Briscoe [29] represented their data for interfacial tension $\sigma$ of fatty alcohols at $\mathrm{W} \mid \mathrm{O}$ as a function of the alcohol concentration $C^{\mathrm{O}}$ in the oil phase. In order to compare these data with results for $\mathrm{W} \mid \mathrm{A}$ (where the surfactant is in the water phase), we recalculate the corresponding concentration $C$ in water by using experimental data for the partition coefficient of the alcohols [30] and for the alcohol's tetramerisation constant in the alkane phase [31]. Aveyard and Mitchell [30] studied the partitioning of alcohols (of chain length $n$ from 4 to 7) between water and various alkanes (subscript $\mathrm{A}$; their carbon number $n_{\mathrm{A}}$ vary from 8 to 16). They found that the partition coefficient in dilute solution, $K_{\mathrm{p}}=C^{\mathrm{O}} / C$, depends on the chain lengths both of the surfactant and the alkane. Their data for $K_{\mathrm{p}}$ followed the linear regression model (cf. footnote 5 in the main text):

$$
\ln K_{\mathrm{p}}=\ln K_{\mathrm{p} 0}-n_{\mathrm{A}} \mu_{\mathrm{A}} / k_{\mathrm{B}} T+n \Delta \mu_{\mathrm{CH}_{2}} / k_{\mathrm{B}} T .
$$

From numerous independent experimental data, we previously found that $\Delta \mu_{\mathrm{CH}_{2}}=1.39 \times k_{\mathrm{B}} T$ [8] (see also the S4). By fitting the data of Aveyard and Mitchell [30] with Eq. (45), we determined $\mu_{\mathrm{A}}=0.057 \times k_{\mathrm{B}} T$ and $\ln K_{\mathrm{p} 0}=-7.05$. These values differ from those of Aveyard and Mitchell [30] by a few percents since they used three fitting parameters $\left(\Delta \mu_{\mathrm{CH}_{2}}\right.$ was considered unknown) instead of our two (fixed $\Delta \mu_{\mathrm{CH}_{2}}=$ $\left.1.39 \times k_{\mathrm{B}} T\right)$. 
In order to calculate the surfactant concentration $C$ in water from the experimental values of $C^{\mathrm{O}}$, we used the relation $C=\gamma^{\mathrm{O}} C^{\mathrm{O}} / K_{\mathrm{p}}$. The activity coefficient $\gamma^{\mathrm{O}}$ in the oil phase was calculated by solving the equation $C^{\mathrm{O}}=\gamma^{\mathrm{O}} C^{\mathrm{O}}+4 K_{1,4}\left(\gamma^{\mathrm{O}} C^{\mathrm{O}}\right)^{4}$, where $K_{1,4}=780 \mathrm{M}^{-3}$ [31,8] is the tetramerisation constant of the alcohol in the oil phase.

In Sec. 3.1, we used the virial equation (6) to fit the tensiometric data and determine the adsorption constant $K_{\mathrm{a}}$ of the alcohols (in Ref. [8], we used instead the HFL model (1), as in Sec. 3.3). We considered only the experimental data of Aveyard and Briscoe which correspond to $\pi^{\mathrm{S}}<10 \mathrm{mN} / \mathrm{m}$ (the first 5-15 points) - in this region, the effect of the ternary interactions can be disregarded so that Eq. (6) is valid (in contrast to the analysis of the complete adsorption models in Sec. 3.3, where all data points available are taken into account). The fit involves two parameters - the adsorption constant $K_{\mathrm{a}}$ and the second virial coefficient $B_{2}$. However, since $B_{2}$ is obtained with rather high dispersion, only the values of $K_{\mathrm{a}}$ are of interest and are discussed in the main text. The results are presented in Fig. 3 as $\ln K_{\mathrm{a}}$ vs. $n$; the regression is illustrated in Fig. 1. The data in Fig. 3 refer to alkane phase varying from octane to hexadecane; unlike the partition coefficient $K_{\mathrm{p}}$ the adsorption constant $K_{\mathrm{a}}$ from water to W|O interface, within the experimental error, seems independent on the length $n_{\mathrm{A}}$ of the alkane molecule. This suggests that the term $-\mu_{\mathrm{A}} n_{\mathrm{A}}$ in Eq. (45) for $K_{\mathrm{p}}$ is related mostly to the state of $\mathrm{OH}$ in the oil phase.

Similar fitting procedure with the virial expansion Eq. (6) has been used with the data for noncohesive surfactants at W|A (N-n-alkyl-N,N-dimethylglycine, $\mathrm{C}_{n} \mathrm{H}_{2 n+1} \mathrm{Me}_{2} \mathrm{~N}^{+} \mathrm{CH}_{2} \mathrm{COO}^{-}$, and short chain length homologues of n-alkyl dimethyl phosphine oxides, $\mathrm{C}_{n} \mathrm{H}_{2 n+1} \mathrm{Me} 2 \mathrm{PO}$ ) and $\mathrm{W} \mid \mathrm{O}$ $\left(\mathrm{C}_{n-1} \mathrm{H}_{2 n-1} \mathrm{COOH}\right.$ with $n=4$ and 5). The results for $K_{\mathrm{a}}$ so obtained are presented in Fig. 3 and Table 1. 


\section{S4. Calculation of the transfer energy $\Delta \mu_{\mathrm{CH}_{3}}$ of the methyl group}

In this supplement, we determine the value of the transfer energy $\Delta \mu_{\mathrm{CH}_{3}}$ of a methyl group from oil to water from the data of Abraham for the solubilities in water of alkanes of different chain lengths $n_{\mathrm{A}}$ (Tables 2 and 3 of Ref. [73]). For the chemical potential of an alkane molecule in the water and alkane phases we use the expressions (cf. Eq. 19-16 of Hill [56]):

$$
\mu^{\mathrm{W}}=\mu_{0}^{\mathrm{W}}+k_{\mathrm{B}} T \ln \frac{x^{\mathrm{W}}}{v^{\mathrm{W}}} ; \quad \mu^{\mathrm{O}}=\mu_{0}^{\mathrm{O}}+k_{\mathrm{B}} T \ln \frac{1}{v^{\mathrm{O}}} .
$$

Here $v^{\mathrm{W}}$ and $v^{\mathrm{O}}$ are molar volumes of water in the water phase and of alkane molecule in the alkane phase respectively; $x^{\mathrm{W}}$ is molar part of the saturated water solution of alkane; $x^{\mathrm{W}} / v^{\mathrm{W}}=C^{\mathrm{W}}$ is the molar concentration of alkane in the water. The standard chemical potentials $\mu_{0}^{\mathrm{W}}$ and $\mu_{0}^{\mathrm{O}}$ involve, first, all internal degrees of freedom of the surfactant molecule, and second, the interaction energy of the molecule with its surroundings (cf. Eq. 19-5 of Hill [56]). Eqs. (46) lead to the following expression for the equilibrium solubility $x^{\mathrm{W}}$ :

$$
\ln x^{\mathrm{w}}=\frac{\mu_{0}^{\mathrm{O}}-\mu_{0}^{\mathrm{w}}}{k_{\mathrm{B}} T}+\ln \frac{v^{\mathrm{w}}}{v^{\mathrm{O}}\left(n_{\mathrm{A}}\right)} .
$$

Assuming that the contributions of the $-\mathrm{CH}_{2}-$ and $-\mathrm{CH}_{3}$ groups are additive (i.e. $\mu_{0}^{\mathrm{W}}-\mu_{0}^{\mathrm{O}}=$ $\left.2 \Delta \mu_{\mathrm{CH}_{3}}+\left(n_{\mathrm{A}}-2\right) \Delta \mu_{\mathrm{CH}_{2}}\right)$, we can rewrite Eq. (47) as follows:

$$
\frac{\Delta \mu_{\mathrm{CH}_{3}}}{k_{\mathrm{B}} T}=-\frac{1}{2} \ln x^{\mathrm{W}}-\frac{n_{\mathrm{A}}-2}{2} \frac{\Delta \mu_{\mathrm{CH}_{2}}}{k_{\mathrm{B}} T}+\frac{1}{2} \ln \frac{v^{\mathrm{w}}}{v^{\mathrm{O}}\left(n_{\mathrm{A}}\right)} .
$$

Here $\Delta \mu_{\mathrm{CH}_{2}}=1.39 \times k_{\mathrm{B}} T$ and $v^{\mathrm{W}}=18.1 \mathrm{~mL} / \mathrm{mol}$; experimental values for $x^{\mathrm{W}}$ and $v^{\mathrm{O}}$ are given in Table $\mathrm{S} 2$, together with the values of $\Delta \mu_{\mathrm{CH}_{3}}$ calculated from Eq. (48).

Tanford [74] did not account for the entropic term $\ln \left(v^{\mathrm{W}} / v^{\mathrm{O}}\right)$ in Eq (48), which is not negligible - it is of the order of 1-2 $k_{\mathrm{B}} T$ and depends on $n_{\mathrm{A}}$ through the molar volume $v^{\mathrm{O}}$. Consequently, he obtained different values both for $\Delta \mu_{\mathrm{CH}_{2}}$ and for $\Delta \mu_{\mathrm{CH}_{3}}$ (his 1.49 vs. our $1.39 \times k_{\mathrm{B}} T$ and his 3.55 vs. our $2.75 \times k_{\mathrm{B}} T$ respectively). The average value for the transfer energy $\Delta \mu_{\mathrm{CH}_{3}}$ for the alkanes in Table S2 is $2.75 \times k_{\mathrm{B}} T$, in agreement with the estimate of Ivanov et al. [46]. 
Table S2. Calculation of the transfer energy $\Delta \mu_{\mathrm{CH}_{3}}$ of a $-\mathrm{CH}_{3}$ from oil to water phase from Eq. (48).

\begin{tabular}{|c|c|c|c|}
\hline$n$ & ${ }^{\mathrm{a}} \ln x^{\mathrm{W}}$ & ${ }^{\mathrm{b}} v^{\mathrm{O}}[\mathrm{mL} / \mathrm{mol}]$ & $\Delta \mu_{\mathrm{CH}_{3}} / k_{\mathrm{B}} T$ \\
\hline 5 & -11.5 & 115 & 2.77 \\
\hline 6 & -13.1 & 132 & 2.8 \\
\hline 7 & -14.4 & 147 & 2.71 \\
\hline 8 & -16.1 & 162 & 2.78 \\
\hline 9 & -17.7 & 179 & 2.85 \\
\hline 10 & -18.9 & 194 & 2.71 \\
\hline 12 & -21.7 & 227 & 2.65 \\
\hline average: & \multicolumn{3}{|l}{} \\
\hline
\end{tabular}

${ }^{\mathrm{a}}$ Data for solubility $x^{\mathrm{W}}$ of liquid alkanes taken from Abraham [73]; ${ }^{\mathrm{b}}$ Data for $v^{\mathrm{O}}$ from Refs. [99,100].

\section{S5. The attraction parameter $\beta$}

In Ref. [15], the expression (21) was used for surfactants with relatively large head groups. For this case, the exponent under the integral can be expanded into series up to the linear term. This leads to an analytical formula for $\beta[15]$ :

$$
\beta=\frac{\pi^{5 / 2}}{32} \frac{n L_{\mathrm{CH}_{2}}}{l_{\mathrm{CH}_{2}} \alpha^{5 / 2} k_{\mathrm{B}} T} \arctan \frac{\pi^{1 / 2} n l_{\mathrm{CH}_{2}}}{2 \alpha^{1 / 2}} .
$$

Even for short chain lengths, Eq. (49) deviates significantly from the exact result (21), cf. Fig. S2. The deviations become larger with the increase of $n$, especially for surfactants of small actual molecular area $\alpha$. Therefore, when the model for $\beta$ is compared with experimental data, we have used the exact result (21) only.

In Fig. S3, the interaction parameter of alcohol films at W|A following from the processing of the experimental data with the SD or the SIAL adsorption models are compared, as functions of the chain length $n$. The SD model works only if the non-linear dependence (21) is used, while the accuracy of SIAL is acceptable only if combined with the linear Eq. (24). 


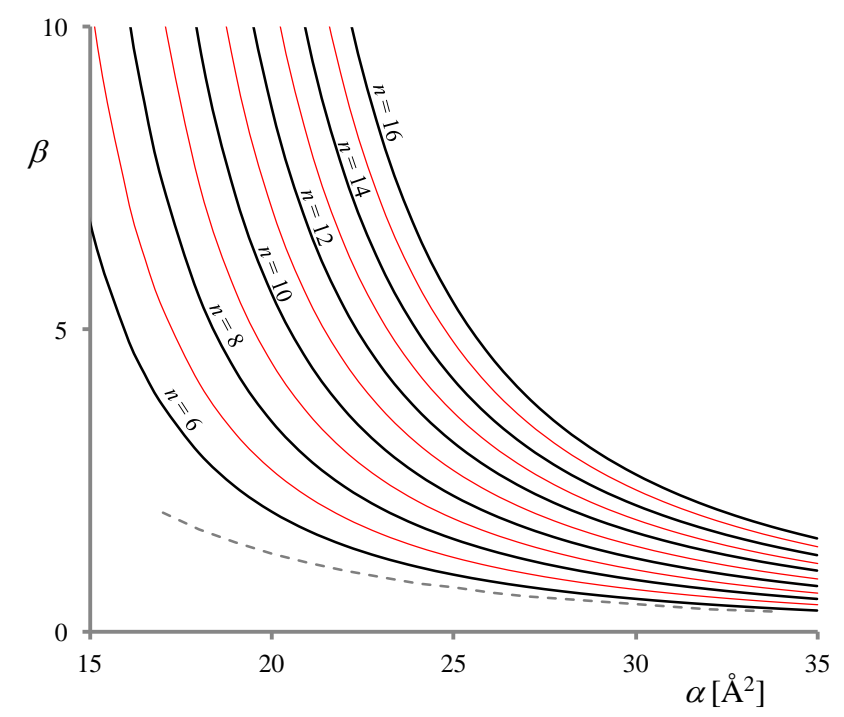

Fig. S2. The attraction parameter $\beta$ vs. the hard-disc area $\alpha$ of the surfactant, calculated by numerical integration of Eq. (21), at various hydrocarbon chain lengths (from $n=6$ to $n=16$; black lines are even values of $n$, red are odd; $\left.25^{\circ} \mathrm{C}\right)$. Dashed line is $\beta(\alpha)$ at $n=6$ calculated according to the linearized formula - it is seen that its accuracy is insufficient.

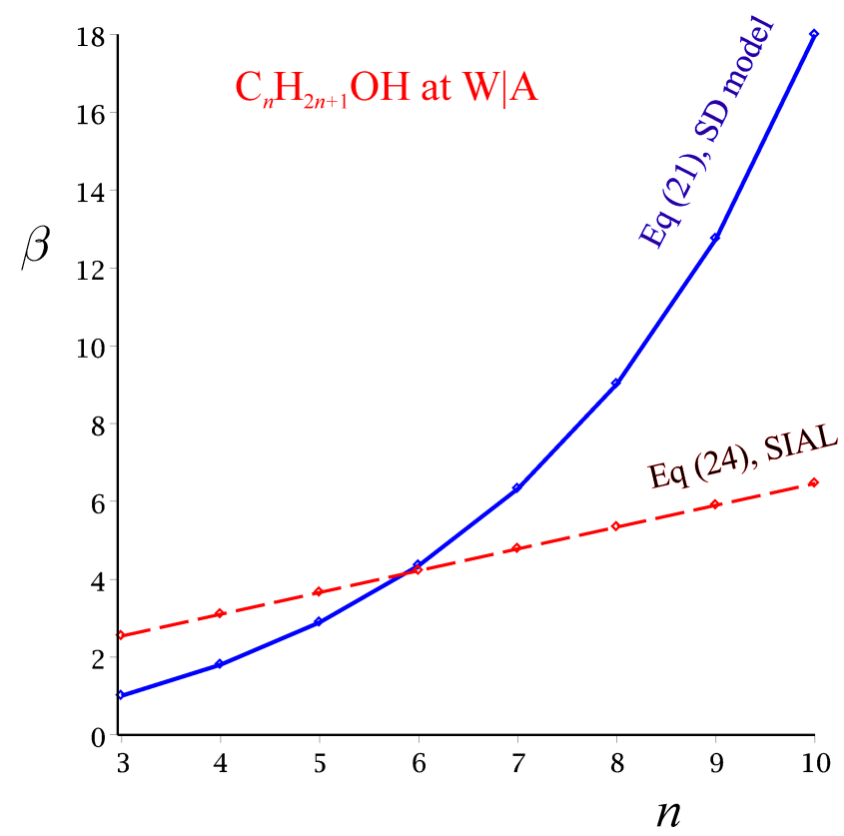

Fig. S3. Attraction parameter $\beta$ of alcohols (from propanol to decanol) at $\mathrm{W} \mid \mathrm{A}$ according to the results from SD and SIAL models. SD model agrees with the tensiometric data for alcohols only in combination with the nonlinear model (21) for $\beta$. Due to the different approach towards the lateral attraction, SIAL model agrees with the tensiometric data only if the linear dependence is used: $\beta=0.86+0.56 n$, see Eq. (24). The combined use of the SD model with Eq. (24), and also of the SIAL model with Eq. (21), strongly disagree with the experiment. 


\section{S6. Comparison of various models and fitting procedures against}

\section{tensiometric data}

Table S3 (for alcohols at $\mathrm{W} \mid \mathrm{O}$ ) and Table S4 (for various amphiphiles at $\mathrm{W} \mid \mathrm{A}$ ) compare all considered model to tensiometric data for whole homologous series of surfactants. The free parameters of the homologous series are determined via the optimization of merit functions similar to Eq. (28). The most important results are discussed in the main text, Sec. 3.3-3.5; those calculated for completeness and for further reference to the parameter values are discussed briefly here and in S8-S10.

An important from practical viewpoint test is the comparison between all models from Sec. 2.1.2 under the same conditions -3 free parameters, $K_{\mathrm{a} 0}, \alpha$ and $\beta$. The results are given in rows $\{8-11\}$ in Table S2. As benchmarks we will use the area $\alpha=16.5 \AA^{2}$ following from crystallographic \& collapse data, the "direct" value of $\ln K_{\mathrm{a} 0}=-21.3$ from Table 1 , and $\beta=0.332$ obtained with the 2-parametric fit of the SD model, which we consider as reliable. The results lead to the following conclusions: (i) the deviations of all 3-parametric models in rows $\{8-11\}$ are exactly the same. (ii) All models yield almost the same value for the adsorption constant $K_{\mathrm{a} 0}$, very close to the one directly determined in Sec. 3.1. This is due to the fact that the initial slope of the $\pi^{\mathrm{S}}(C)$ curve (the Henry region) is model-independent. (iii) The SD model yields values of $\alpha$ and $\beta$ closest to the benchmark values. (iv) The model of SIAL also gives a value of $\alpha$ close to $16.5 \AA^{2}$ but slightly higher $\beta$ than SD. (v) The models of van der Waals and Frumkin give considerably larger values of $\alpha$ (better for vdW), and negative values of $\beta$ as can be expected from Eqs. (19). 
Table S3. Adsorption parameters of $\mathrm{C}_{n} \mathrm{H}_{2 n+1} \mathrm{OH}$ at $\mathrm{W} \mid \mathrm{O}$ interfaces, obtained by the minimization of a merit function of the type of Eq. (28).

\begin{tabular}{|c|c|c|c|c|}
\hline $\begin{array}{c}\mathrm{C}_{n} \mathrm{H}_{2 n+1} \mathrm{OH} \\
\text { at water|alkane }\end{array}$ & $\ln \left(K_{\mathrm{a} 0} /[\mathrm{m}]\right)$ & $\begin{array}{c}\alpha, \alpha_{\mathrm{v}}, \alpha_{\mathrm{L}} \\
{\left[\AA^{2}\right]}\end{array}$ & $\beta, \beta_{\mathrm{V}}, \beta_{\mathrm{L}}$ & $\begin{array}{c}\mathrm{dev} \\
{[\mathrm{mN} / \mathrm{m}]}\end{array}$ \\
\hline \multicolumn{5}{|c|}{ one-parametric fit } \\
\hline$\{1\} \mathrm{HFL}$ & -20.95 & $16.5^{\mathrm{b}}$ & $\underline{0}$ & 0.49 \\
\hline \multicolumn{5}{|c|}{ two-parametric fits } \\
\hline$\{2\} \mathrm{HFL}$ & -21.1 & 14.2 & $\underline{0}$ & 0.40 \\
\hline$\{3\}$ Volmer & -21.2 & 24.4 & $\underline{0}$ & 0.39 \\
\hline$\{4\}$ Langmuir & -21.25 & 39.0 & $\underline{0}$ & 0.40 \\
\hline$\{5\} \mathrm{SD}$ & -21.1 & $16.5^{\mathrm{b}}$ & 0.332 & 0.40 \\
\hline$\{6\} \mathrm{vdW}$ & -21.0 & 18.2 & $-1^{\mathrm{c}}$ & 0.40 \\
\hline$\{7\}$ Frumkin & -21.0 & 21.0 & $-3 / 2^{\mathrm{c}}$ & 0.41 \\
\hline \multicolumn{5}{|c|}{ three-parametric fits } \\
\hline$\{8\}$ SD & -21.1 & 17.2 & 0.437 & 0.39 \\
\hline$\{9\}$ SIAL & -21.2 & 15.8 & 0.556 & 0.39 \\
\hline$\{10\} \mathrm{vdW}$ & -21.2 & 24.2 & -0.032 & 0.39 \\
\hline$\{11\}$ Frumkin & -21.1 & 35.1 & -0.297 & 0.39 \\
\hline
\end{tabular}

For each model, the assumed fixed values of the parameters are underlined. The transfer energy in the expression (25) for the adsorption constant is fixed to $\Delta \mu_{\mathrm{CH}_{2}}=1.39 \times k_{\mathrm{B}} T$ for all models. The equations defining the adsorption models (first column) are listed in S1. ${ }^{a}$ Alcohols are with chain length $n=8 \div 18$ (even $n$ only), oil phase is alkane with $n_{\mathrm{A}}=8 \div 16 ; T=20^{\circ} \mathrm{C}$. ${ }^{\mathrm{b}}$ Value of the area, calculated from data for the crystallographic and collapse area of alcohols. ${ }^{\mathrm{c}}$ In accordance with Eqs (19), the fixed values $\beta_{\mathrm{L}}=-3 / 2$ and $\beta_{\mathrm{V}}=-1$ correspond to the lack of attraction $(\beta=0)$. 
Table S4. Adsorption parameters of $\mathrm{C}_{n} \mathrm{H}_{2 n+1} \mathrm{OH}, \mathrm{C}_{n-1} \mathrm{H}_{2 n-1} \mathrm{COOH}, \mathrm{C}_{n} \mathrm{H}_{2 n+1} \mathrm{Me}_{2} \mathrm{PO}$ and $\mathrm{C}_{n} \mathrm{H}_{2 n+1} \mathrm{Me}_{2} \mathrm{~N}^{+} \mathrm{CH}_{2} \mathrm{COO}^{-}$ at W|A, obtained by minimization of a merit function of the type of Eq. (28).

\begin{tabular}{|c|c|c|c|c|c|c|}
\hline model & $\begin{array}{l}\text { num. free } \\
\text { parameters }\end{array}$ & range of $n^{\mathrm{a}}$ & $\ln \left(K_{\mathrm{a} 0} /[\mathrm{m}]\right)^{\mathrm{b}}$ & $\begin{array}{c}\alpha, \alpha_{\mathrm{V}}, \alpha_{\mathrm{L}} \\
{\left[\AA^{2}\right]}\end{array}$ & $\beta, \beta_{\mathrm{V}}, \beta_{\mathrm{L}}$ & $\begin{array}{c}d e v \\
{[\mathrm{mM} / \mathrm{m}]}\end{array}$ \\
\hline \multicolumn{7}{|c|}{$\mathrm{C}_{n} \mathrm{H}_{2 n+1} \mathrm{Me}_{2} \mathrm{P}^{+} \mathrm{O}^{-}$at $\mathrm{W} \mid \mathrm{A}, \quad n=7-16, \quad$ average $T=23.5^{\circ} \mathrm{C}$} \\
\hline$\{1\}$ SD & 1 & 7-11/non-coh & -19.9 & $\underline{29}^{\mathrm{c}}$ & Eq. $(21)^{\mathrm{d}}$ & 1.0 \\
\hline$\{2\}$ SD & 2 & 7-11/non-coh & -20.0 & 28.2 & Eq. $(21)^{\mathrm{d}}$ & 0.98 \\
\hline$\{3\}$ SD & 2 & 7-11/non-coh & -21.0 & $\underline{29}^{c}$ & $\underline{0.49(n+1)^{\mathrm{e}}}$ & 1.7 \\
\hline$\{4\} \mathrm{SD}$ & 1 & 7-16/all & -19.7 & $\underline{29^{c}}$ & Eq. $(21)^{\mathrm{d}}$ & 2.0 \\
\hline$\{5\}$ SIAL & 1 & 7-11/non-coh & -20.5 & $29^{c}$ & $0.49(n+1)^{\mathrm{e}}$ & 1.7 \\
\hline$\{6\}$ SIAL & 3 & 7-11/non-coh & -20.5 & $\underline{29}^{\mathrm{c}}$ & $-0.08+0.35 n$ & 1.0 \\
\hline$\{7\}$ SIAL & 3 & 7-16/all & -20.6 & $29^{c}$ & $-0.08+0.38 n$ & 1.3 \\
\hline$\{8\} \mathrm{vdW}$ & 4 & 7-11/non-coh & -19.9 & 33.0 & $-2.46+0.273 n$ & 0.91 \\
\hline$\{9\}$ Frumkin & 4 & 7-11/non-coh & -19.8 & 43.4 & $-2.41+0.206 n$ & 0.93 \\
\hline \multicolumn{7}{|c|}{$\mathrm{C}_{n} \mathrm{H}_{2 n+1} \mathrm{Me}_{2} \mathrm{~N}^{+} \mathrm{CH}_{2} \mathrm{COO}^{-}$at $\mathrm{W} \mid \mathrm{A}, \quad n=8-16, \quad$ average $T=20^{\circ} \mathrm{C}$} \\
\hline$\{10\} \mathrm{SD}$ & 2 & 8-16/non-coh & -21.3 & 31.0 & Eq. $(21)^{\mathrm{d}}$ & 1.8 \\
\hline$\{11\} \mathrm{SD}$ & 3 & 8-16/non-coh & -21.6 & 30.2 & 2.31 & 1.1 \\
\hline$\{12\}$ SIAL & 4 & 8-16/non-coh & -21.55 & 23.4 & $1.93+0 n$ & 1.1 \\
\hline \multicolumn{7}{|c|}{$\mathrm{C}_{n-1} \mathrm{H}_{2 n-1} \mathrm{COOH}$ at W|A, $n=3-10, \quad$ average $T=21^{\circ} \mathrm{C}$} \\
\hline$\{13\} \mathrm{SD}$ & 1 & 3-4/non-coh & -20.4 & $\underline{18}^{\mathrm{c}}$ & Eq. $(21)^{\mathrm{d}}$ & 0.57 \\
\hline$\{14\} \mathrm{SD}$ & 1 & 3-10/all & -20.2 & $\underline{18^{\mathrm{c}}}$ & Eq. $(21)^{\mathrm{d}}$ & 0.93 \\
\hline$\{15\}$ SD & 2 & 3-10/all & -20.2 & 18.2 & Eq. $(21)^{\mathrm{d}}$ & 0.92 \\
\hline$\{16\}$ SIAL & 1 & 3-10/all & -20.4 & $18^{\mathrm{c}}$ & $(n+1) 0.49^{\mathrm{e}}$ & 0.94 \\
\hline$\{17\}$ SIAL & 3 & 3-10/all & -20.7 & $\underline{18}^{\mathrm{c}}$ & $1.44+0.46 n$ & 0.78 \\
\hline$\{18\}$ vdW & 4 & $3-10 /$ all & -20.4 & 22.55 & $-0.47+0.35 n$ & 0.63 \\
\hline$\{19\}$ Frumkin & 4 & 3-10/all & -20.1 & 28.8 & $-1.0+0.26 n$ & 0.70 \\
\hline \multicolumn{7}{|c|}{$\mathrm{C}_{n} \mathrm{H}_{2 n+1} \mathrm{OH}$ at $\mathrm{W} \mid \mathrm{A}, \quad n=3-10, \quad T=21^{\circ} \mathrm{C}$} \\
\hline$\{20\}$ SD & 2 & 3-4/non-coh & -20.1 & $\underline{16.5}^{\mathrm{c}}$ & Eq. $(21)^{\mathrm{d}}$ & 0.88 \\
\hline$\{21\} \mathrm{SD}$ & 1 & 3-10/all & -20.4 & $16.5^{\mathrm{c}}$ & Eq. $(21)^{\mathrm{d}}$ & 1.44 \\
\hline$\{22\} \mathrm{SD}$ & 2 & 3-10/all & -20.1 & 17.3 & Eq. $(21)^{\mathrm{d}}$ & 1.06 \\
\hline$\{23\}$ SIAL & 1 & $3-10 /$ all & -20.1 & $16.5^{\mathrm{c}}$ & $(n+1) 0.49^{\mathrm{e}}$ & 1.5 \\
\hline$\{24\}$ SIAL & 3 & 3-10/all & -20.6 & $16.5^{\mathrm{c}}$ & $0.86+0.56 n$ & 0.95 \\
\hline$\{25\} \mathrm{vdW}$ & 4 & $3-10 /$ all & -20.3 & 20.9 & $-0.48+0.39 n$ & 1.1 \\
\hline$\{26\}$ Frumkin & 4 & 3-10/all & -21.6 & 31.0 & $0.97+0.26 n$ & 1.6 \\
\hline
\end{tabular}

For each model, the assumed fixed values of the parameters are underlined. The equations defining the adsorption models (first column) are listed in S1. ${ }^{a}$ Data either only for non-cohesive or for all (cohesive and non-cohesive) homologues are used. ${ }^{\mathrm{b}}$ For $\mathrm{W} \mid \mathrm{A}$, we use fixed value of the transfer energy $\Delta \mu_{\mathrm{CH}_{2}}=1.04 \times k_{\mathrm{B}} T$. ${ }^{\mathrm{c}}$ Fixed value of $\alpha$ calculated from the crystallographic and/or collapse areas. ${ }^{\mathrm{d}}$ Fixed to the value predicted by the nonlinear Eq. (21). Note that the expression (21) for $\beta$ involves $\alpha$ as a parameter; this was accounted for in the optimization procedure. ${ }^{\mathrm{e}}$ Fixed value of $\beta_{1}=0.49$ taken from Smith [63]. 


\section{S7. Analysis of the dispersion of the SD model as a function of the interaction}

\section{parameters $\alpha$ and $\beta$}

The two interaction parameters $\alpha$ and $\beta$ affect the surface pressure isotherm in similar manner and in result they cannot be determined from tensiometric data with good accuracy in most cases. This is valid for all models from Sec. 2.1.2. To illustrate the problem, we will consider the deviation of the SD model, Eqs. (10)-(12), from the data for oil-soluble alcohols (Eq. (28) and row \{8\} from Table S3). We fixed $\ln \left(K_{\mathrm{a} 0} /[\mathrm{m}]\right)$ to its best value, -21.14 , and then analysed the merit function $\operatorname{dev}(\alpha, \beta)$. This function has a minimum at $\alpha=17.2 \AA^{2}$ and $\beta=0.437$; the optimal value of $\operatorname{dev}$ is $0.392 \mathrm{mN} / \mathrm{m}$. It turns out, however, that the minimum of $\operatorname{dev}(\alpha, \beta)$ is rather flat. The deviation is within $1 \%$ of the optimal value for any $\beta$ between 0 and 1 and $\alpha$ between 14 and $21 \AA^{2}$, provided that they are related between each other as $\beta=$ $0.13 \alpha-1.8$ (Fig. S4). This problem is a significant source of errors, especially if data for each homologue is fitted separately. Therefore, fits with both $\alpha$ and $\beta$ being left as adjustable parameters must be avoided. This means, for example, that the result for $\beta$ from row $\{5\}$ in Table S3 (2-parametric SD model with free $K_{\mathrm{a} 0}$ and $\beta$ ) is far more reliable than the one in row \{8\} (3-parametric SD model with free $K_{\mathrm{a} 0}, \alpha$ and $\beta$ ).

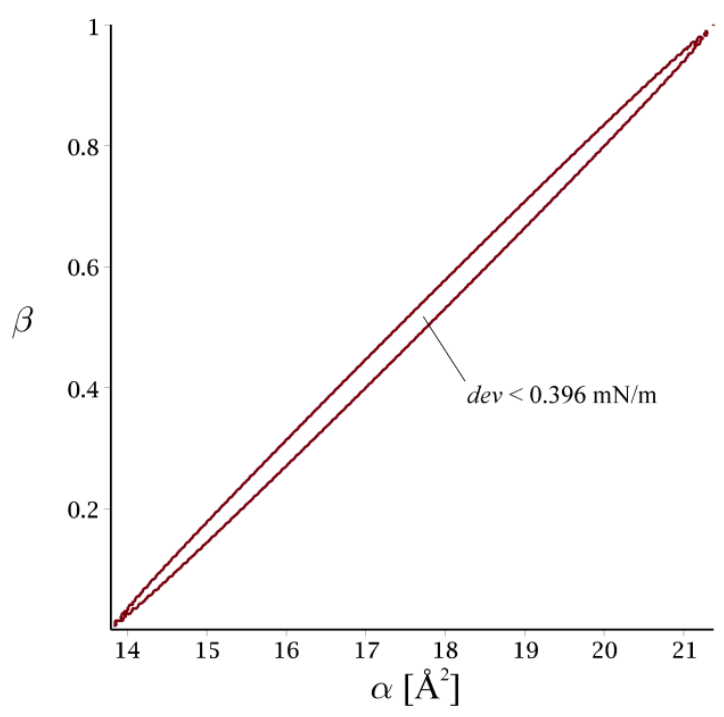

Fig. S4. Standard deviation of the SD model compared to the tensiometric data for oil-soluble alcohols $(n=$ $8 \div 18)$ at the $\mathrm{W} \mid \mathrm{O}$ interface as a function of $\alpha$ and $\beta$. Fixed value $\ln \left(K_{\mathrm{a} 0} /[\mathrm{m}]\right)=-21.14$ is used. It is seen that any value of $\beta$ between 0 and 1 and any $\alpha$ between 14 and $21 \AA^{2}$ can give deviation below $0.396 \mathrm{mN} / \mathrm{m}$ (the global minimum is by $1 \%$ smaller; any set of adsorption parameters falling inside the ellipse has $d e v<0.396 \mathrm{mN} / \mathrm{m}$ ). 


\section{S8. Adsorption of the water-soluble non-ionic surfactants at the $\mathrm{W} \mid \mathrm{O}$}

\section{interface}
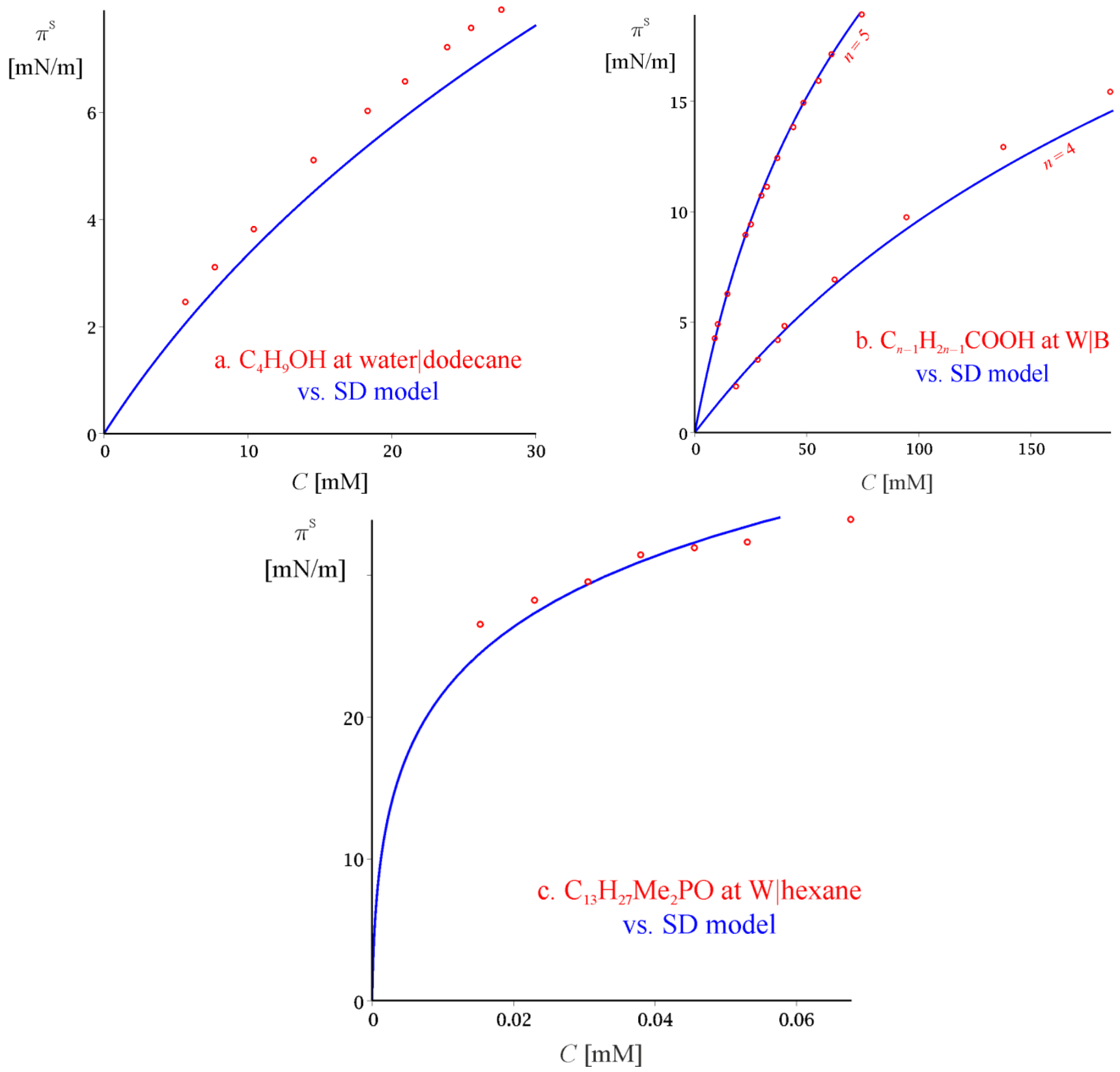

Fig. S5. Interfacial pressure $\pi^{\mathrm{S}}$ vs. concentration $C$ in the water of several water-soluble surfactants at $\mathrm{W} \mid \mathrm{O}$.

a. Butanol at water|dodecane, $T=20^{\circ} \mathrm{C}$. Red points: data from Ref. [79]; blue line: SD model with no fitting parameters (the values of the long-chained oil-soluble alcohols from Table 2 were used). b. Butanoic and pentanoic acid at water|benzene, $35^{\circ} \mathrm{C}$. Red points: data from Ref. [69]; blue lines: SD model with $K_{\mathrm{a} 0}=2.00 \AA$ and $\beta=1.56$. c. Tridecyldimethylphosphineoxide at water|hexane, $20^{\circ} \mathrm{C}$. Red points: data from Ref. [80]; blue lines: SD model with $K_{\mathrm{a} 0}=1.63 \AA$ and $\beta \approx 0$. 
The findings presented in Sec. 3.3 were about oil-soluble alcohols. In order to verify the obtained results, we analysed also the interfacial tension data for a water-soluble alcohol. To the best of our knowledge, only data for butanol, adsorbed at water|dodecane interface are available [79]. We used the SD model with fixed values of the actual area per molecule, $\alpha=16.5 \AA^{2}$, and $\beta=0.332$; the adsorption constant is $K_{\mathrm{a}}=K_{\mathrm{a} 0} \exp \left(n \Delta \mu_{\mathrm{CH} 2} / k_{\mathrm{B}} T\right)=0.169 \mu \mathrm{m}$ following from the value $\ln \left(K_{\mathrm{a} 0} /[\mathrm{m}]\right)=-21.14$ (Table 2). The predicted surface tension isotherm is in good agreement with the experimental data, Fig. S5a. The small positive deviation of the data is probably due to the non-ideality of the aqueous butanol solution, and perhaps the inaccuracy of Eq. (45) for $K_{\mathrm{p}}$ (which can shift the $\ln K_{\mathrm{a} 0}$ values determined for the oil-soluble surfactants in Table 2 by an additive constant).

The tensiometric data for butanoic and pentanoic acids at the water|benzene (W|B) interface were processed with the 2-parametric SD model ( $\beta$ is assumed independent of $n$ and $\ln K_{\mathrm{a}}$ is assumed to follow Eq. (25) with $\Delta \mu_{\mathrm{CH}_{2}}=1.39 \times k_{\mathrm{B}} T$ as with alcohols at $\mathrm{W} \mid \mathrm{O}$, cf. Sec. 3.3). The result is illustrated in Fig. $\mathrm{S} 5 \mathrm{~b}$. Data for a single homologue of the phosphineoxides at $\mathrm{W} \mid \mathrm{O}$ is available - it is fitted with the SD model in Fig. S5c. The results are discussed in Sec. 3.3.

\section{S9. Application of the adsorption models to data for $\mathrm{N}$-alkyl-N,N- \\ dimethylglycines at $\mathrm{W} \mid \mathbf{A}$}

All homologues of the zwitterionic $\mathrm{C}_{n} \mathrm{H}_{2 n+1} \mathrm{Me}_{2} \mathrm{~N}^{+} \mathrm{CH}_{2} \mathrm{COO}^{-}$at W|A from Refs. $[93,94,83](n=8-$ 16) point at weakly cohesive behaviour, suggesting that $\beta$ is close to 2 (cf. Sec. 3.5). Unfortunately, we found no reliable data for the area per molecule of these surfactants. In addition, the data showed significant disagreement with both Eq. (21) and Eq. (23) for $\beta$. This is evident from the high deviation of the SD model with $\beta$ fixed to the predictions of Eq. (21) $(1.8 \mathrm{mN} / \mathrm{m}$, cf. row $\{10\}$ in Table S4). A 4parametric fit with SIAL model with assumed linear $\beta(n)$ dependence (Eqs. (8),(9)\&(24) with parameters

$K_{\mathrm{a} 0}, \alpha, \beta_{0}$ and $\beta_{1}$ ) yields $\beta_{1}=0$, which means essentially that the attraction parameter $\beta$ is almost independent of $n$ (row $\{12\}$ in Table S4). We performed two more tests of this result. The first one was to set $\beta=$ const in the SD model for all $\mathrm{C}_{n} \mathrm{H}_{2 n+1} \mathrm{Me}_{2} \mathrm{~N}^{+} \mathrm{CH}_{2} \mathrm{COO}^{-}$homologues. This yields a relatively low deviation of $1.1 \mathrm{mN} / \mathrm{m}$ and area per molecule close to that of $\mathrm{C}_{n} \mathrm{H}_{2 n+1} \mathrm{Me}_{2} \mathrm{PO}$ (row $\{11\}$ in Table $\mathrm{S} 4)$. The second test is following from the fact that if $\beta$ is not strongly dependant on $n$, then scaling behaviour similar to the one shown in Fig. 4 for the alcohols at $\mathrm{W} \mid \mathrm{O}$ can be expected. Indeed, the SD 
isotherm (12) can be written as:

$$
K_{\mathrm{a} 0} C \exp \frac{n \Delta \mu_{\mathrm{CH}_{2}}}{k_{\mathrm{B}} T}=\frac{\Gamma}{1-\alpha \Gamma}\left(\frac{2}{1+R_{\beta}}\right)^{2+1 / \beta} \exp \left[\frac{\alpha \Gamma(4-3 \alpha \Gamma)}{(1-\alpha \Gamma)^{2}} \times \frac{2}{1+R_{\beta}}\right],
$$

which is similar to Eq. (29). Provided that $\beta$ is independent of $n$, Eq. (50) suggests that if $\pi^{\mathrm{S}}$ is plotted against $C \times \exp \left(n \Delta \mu_{\mathrm{CH}_{2}} / k_{\mathrm{B}} T\right)$, where the value $\Delta \mu_{\mathrm{CH}_{2}} / k_{\mathrm{B}} T=1.04$ for $\mathrm{W} \mid \mathrm{A}$ is used, data for all homologues must fall on a single master curve. This is demonstrated in Fig. S6. Only the most long-chained homologues deviate from the theoretical line predicted by the SD model with constant $\beta$.

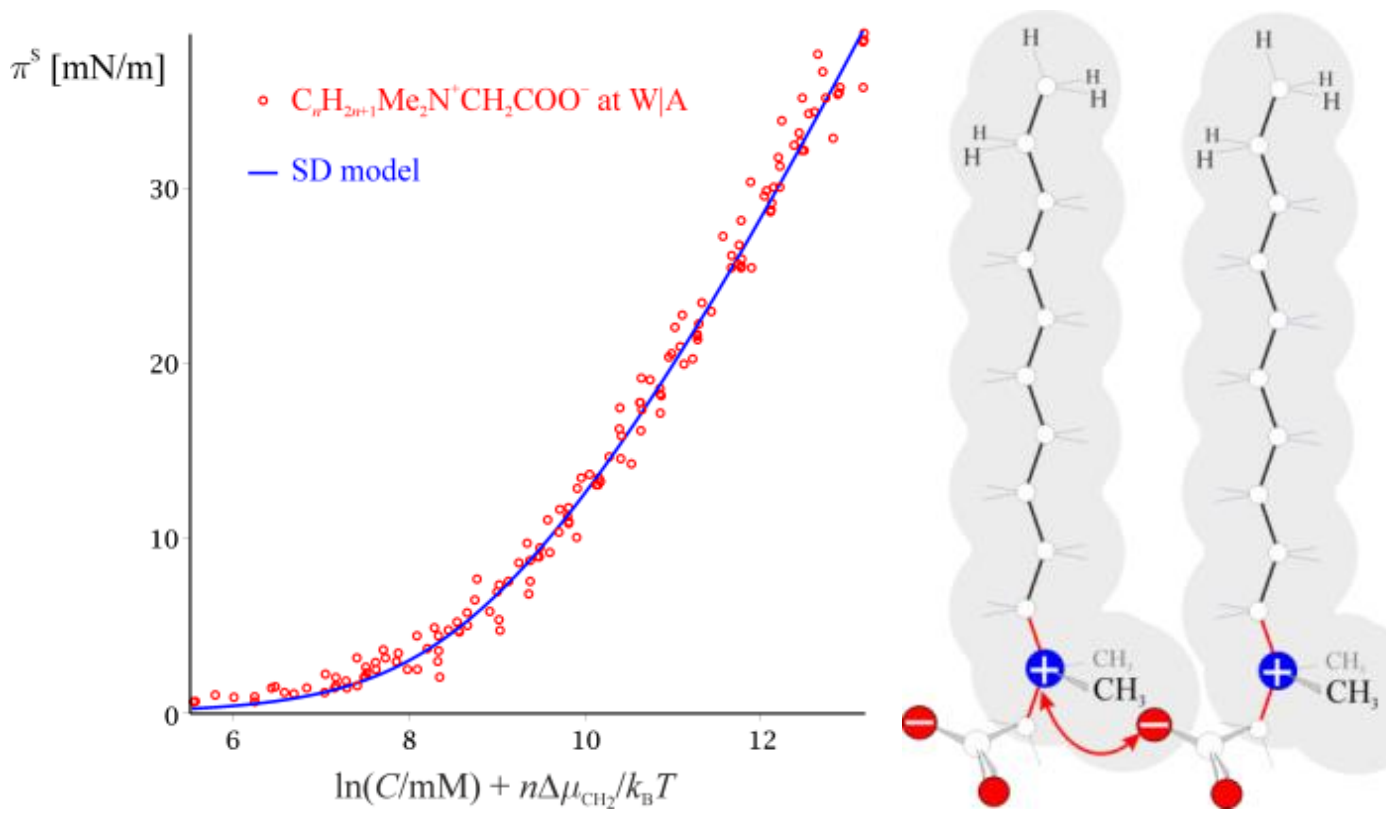

Fig. S6. Surface pressure $\pi^{\mathrm{S}}$ vs. scaled concentration of N-alkyl-N,N-dimethylglycines at W|A $(n=8-16, T=$

$\left.20^{\circ} \mathrm{C}\right)$. Data for all homologues fall on a single master curve when plotted in $\pi^{\mathrm{S}} \mathrm{vs} . \ln \left[C \times \exp \left(n \Delta \mu_{\mathrm{CH}_{2}} / k_{\mathrm{B}} T\right)\right]$ coordinates (with $\Delta \mu_{\mathrm{CH}_{2}} / k_{\mathrm{B}} T=1.04$ ), in agreement with Eq. (50). The line is calculated via the SD model, Eqs. (10)-(12), with $K_{\mathrm{a} 0}=4.33 \AA, \alpha=30.2 \AA^{2}$ and $\beta=2.31$ (Table 2). The scaling demonstrates that $\beta$ is independent of $n$. This fact can be explained with the strong electrostatic attraction between the head groups (illustrated with a red arrow between the positively charged $\mathrm{N}$ - and the negatively charged $\mathrm{O}$-atoms in the picture on the right) seemingly, this interaction dominates over the van der Waals attraction between the hydrocarbon chains.

A possible explanation of the observed little or no dependence of $\beta$ on $n$ is the following one. Due to the high area per molecule, the attraction between the hydrophobic chains is relatively small, cf. Fig. 2. On the other hand, a very strong electrostatic attraction is possible between the head groups, illustrated in Fig. S6 with a red arrow. If the latter interaction is prevailing (which seems to be true for all homologues with the exception of those with the longest chains), $\beta$ will indeed depend only on the head group. 


\section{S10. Phase transition in the continual approach - the $\pi^{\mathrm{S}}(C)$ isotherm}

In this Supplement, the phase behaviour predicted by the SIAL model will be considered in some detail.

The set of extremum points of Eq. (8) for $\pi^{\mathrm{S}}(I)$ at any value of $\beta$ corresponds to the spinodal curve of this EOS, i.e. the spinodal is defined with the condition for extremum, $\partial \pi^{\mathrm{s}}(\Gamma ; \beta) / \partial \Gamma=0$. Substituting here the expression (8) for $\pi^{\mathrm{S}}$ of the SIAL model and solving, we can find the relation between $\beta$ and $\Gamma$ defining the spinodal:

$$
\beta_{\text {spinodal }}=\frac{1+\alpha \Gamma}{2 \alpha \Gamma(1-\alpha \Gamma)^{3}} .
$$

Substituting $\beta$ with $\beta_{\text {spinodal }}$ in the EOS (8), we obtain the spinodal curve in $\pi^{\mathrm{S}}$ vs. $\Gamma$ coordinates:

$$
\frac{\alpha \pi_{\text {spinodal }}^{\mathrm{s}}}{k_{\mathrm{B}} T}=\frac{\alpha \Gamma(1-3 \alpha \Gamma)}{2(1-\alpha \Gamma)^{3}} .
$$

The spinodal curve is plotted in Fig. S7a. Below the spinodal curve, no stable phase exists (the mechanical condition for stability, $\partial \pi^{\mathrm{S}}(\Gamma) / \partial \Gamma>0$, is violated). Between the spinodal and the binodal, only metastable gaseous (on the right of the spinodal) and LE (on the left) phases exist; normally, these metastable phases must pass through a phase transition, forming a heterogeneous film.

To represent the spinodal in $\pi^{\mathrm{S}}$ vs. $C$ coordinates, we substitute Eq. (51) into SIAL adsorption isotherm (9):

$$
\alpha K_{\mathrm{a}} C_{\text {spinodal }}=\frac{\alpha \Gamma}{1-\alpha \Gamma} \exp \left[\frac{-1+2 \alpha \Gamma-5 \alpha^{2} \Gamma^{2}+2 \alpha^{3} \Gamma^{3}}{(1-\alpha \Gamma)^{3}}\right] .
$$

Eqs. (52)-(53) define parametrically the spinodal in $\pi^{\mathrm{s}}$ vs. $C$ (with parameter $\alpha \Gamma$ ). The result is shown in Fig. S7b-d (red dash-dot line). The cusp of the spinodal corresponds to the coordinates of the critical point.

The SIAL model gives no analytical expression for the binodal curve. The binodal in Fig. S7a is the numerical solution of the conditions for mechanical and chemical equilibrium between the gaseous and the LE phase, $\pi^{\mathrm{S}}\left(\Gamma^{\mathrm{G}} ; \beta\right)=\pi^{\mathrm{S}}\left(\Gamma^{\mathrm{LE}} ; \beta\right)$ and $\gamma^{\mathrm{S}}\left(\Gamma^{\mathrm{G}} ; \beta\right) \Gamma^{\mathrm{G}}=\gamma^{\mathrm{S}}\left(\Gamma^{\mathrm{LE}} ; \beta\right) \Gamma^{\mathrm{LE}}$, where the functions $\pi^{\mathrm{S}}$ and $\gamma^{\mathrm{S}}$ are given by Eqs. (8) and by SIAL's surface activity coefficient,

$$
\ln \gamma^{\mathrm{s}}=-\ln (1-\alpha \Gamma)+\frac{\alpha \Gamma(3-2 \alpha \Gamma)}{(1-\alpha \Gamma)^{2}}-2 \beta \alpha \Gamma .
$$

compare to Eq. (2). The equilibrium conditions are solved for $\Gamma^{\mathrm{G}}$ and $I^{\mathrm{LE}}$ for each $\beta>\beta_{\text {cr. }}$ The obtained 
$I^{\mathrm{G}}$ and $\Gamma^{\mathrm{LE}}$ are then substituted in Eqs. (8) and (9) to calculate $\pi_{\text {binodal }}^{\mathrm{S}}$ and $C_{\text {binodal }}$ corresponding to the binodal curve (at the binodal, it follows from the condition for chemical equilibrium that $C^{\mathrm{G}}=C^{\mathrm{LE}} \equiv$ $C_{\text {binodal }}$ and from the mechanical equilibrium that $\pi^{\mathrm{S}, \mathrm{G}}=\pi^{\mathrm{S}, \mathrm{LE}} \equiv \pi_{\text {binodal }}^{\mathrm{S}}$ ). The result is plotted in Fig. S7a $\left(\pi_{\text {binodal }}^{\mathrm{S}}\right.$ vs. $1 / \Gamma^{\mathrm{G}}$ at $\Gamma<\Gamma_{\mathrm{cr}}$ and $\pi_{\text {binodal }}^{\mathrm{S}}$ vs. $1 / \Gamma^{\mathrm{LE}}$ at $\left.\Gamma>\Gamma_{\mathrm{cr}}\right)$ and Fig. S7b-d (blue dotted line, $\pi_{\text {binodal }}^{\mathrm{S}}$ vs. $\left.C_{\text {binodal }}\right)$. The coexistence curve in $\pi^{\mathrm{S}}$ vs. $C$ coordinates has no two branches and it ends at the critical point.

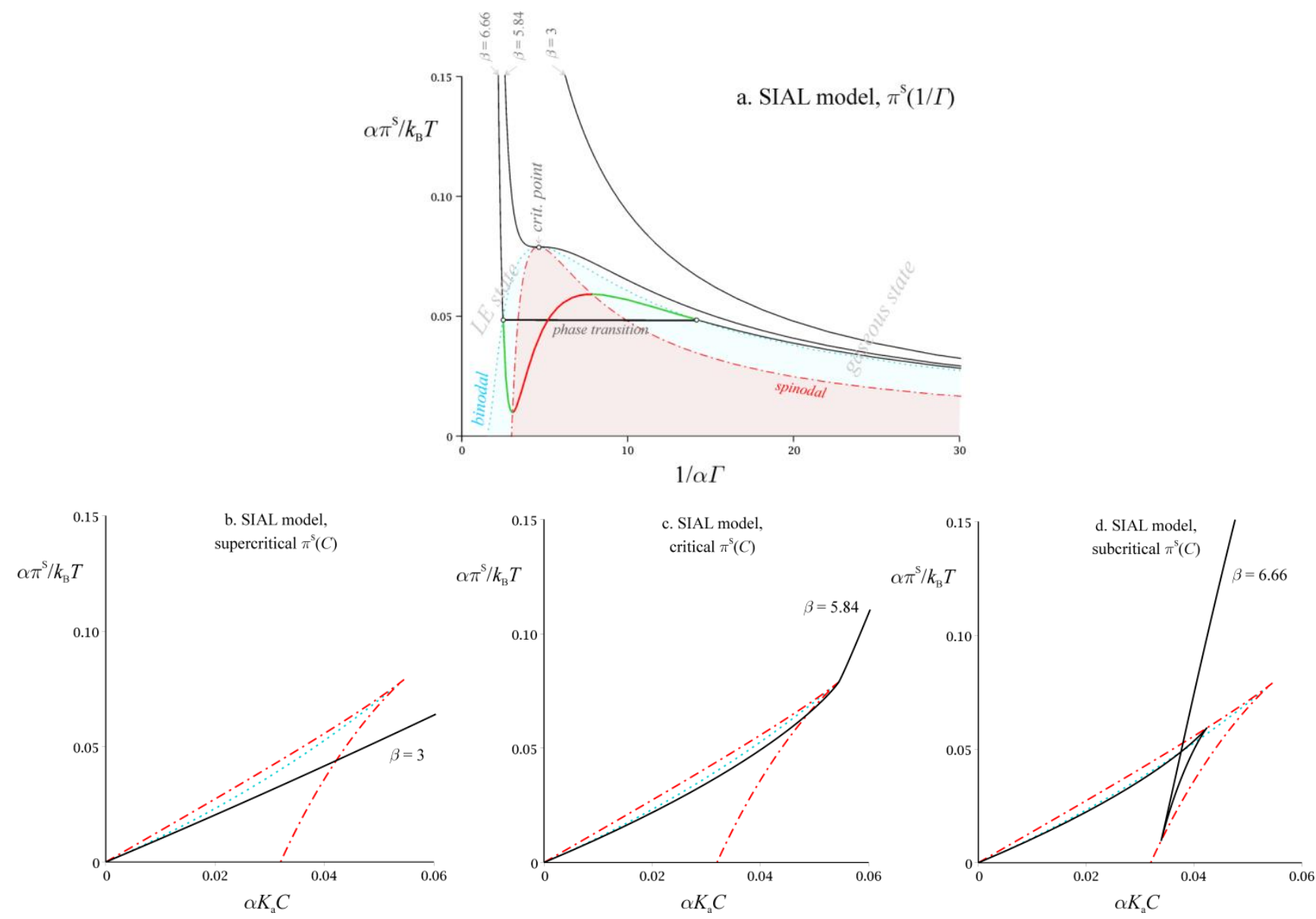

Fig. S7. a. Phase diagram in $\pi^{\mathrm{S}}$ vs. $1 / \Gamma$ coordinates (suitably made dimensionless) according to SIAL model, including the spinodal. Below the spinodal stands the truly unstable 2-D fluid. Between the spinodal and the binodal are the metastable gaseous and LE phases. b-d. Phase diagram in $\pi^{\mathrm{S}}$ vs. $C$ coordinates (suitably made dimensionless) for a supercritical, critical and subcritical adsorption layers. Above the critical $\beta$, the $\pi^{\mathrm{S}}(C)$ curve has a characteristic intersection point with itself. The intersection point is falling on the binodal and corresponds to the gaseous-LE phase transition. The two cusps falling on the spinodal mark the boundaries of the stable gaseous and LE phase. The branch connecting the two cusps correspond to the unstable state of the layer (corresponding to the part of the $\pi^{\mathrm{S}}(1 / \Gamma)$ below the spinodal in a). 


\section{Additional references}

98 S.J. Rehfeld, J. Phys. Chem., 1967, 71, 738.

99 D.R. Lide (editor), CRC Handbook of Chemistry and Physics, $89^{\text {th }}$ ed. CRC Press/Taylor and Francis, Boca Raton; 2009.

100 R.W. Gallant, C.L. Yaws, Physical properties of hydrocarbons, Vol. 1, 2nd ed. Gulf Publishing Company; 1992. 